\title{
Microbiota, obesity and insulin resistance
}

Citation for published version (APA):

Reijnders, D. (2016). Microbiota, obesity and insulin resistance: unraveling the impact of the microbiome on metabolic health in humans. [Doctoral Thesis, Maastricht University]. Proefschriftmaken.nl II Uitgeverij Boxpress. https://doi.org/10.26481/dis.20160921dr

Document status and date:

Published: 01/01/2016

DOI:

10.26481/dis.20160921dr

Document Version:

Publisher's PDF, also known as Version of record

\section{Please check the document version of this publication:}

- A submitted manuscript is the version of the article upon submission and before peer-review. There can be important differences between the submitted version and the official published version of record.

People interested in the research are advised to contact the author for the final version of the publication, or visit the DOI to the publisher's website.

- The final author version and the galley proof are versions of the publication after peer review.

- The final published version features the final layout of the paper including the volume, issue and page numbers.

Link to publication

\footnotetext{
General rights rights.

- You may freely distribute the URL identifying the publication in the public portal. please follow below link for the End User Agreement:

www.umlib.nl/taverne-license

Take down policy

If you believe that this document breaches copyright please contact us at:

repository@maastrichtuniversity.nl

providing details and we will investigate your claim.
}

Copyright and moral rights for the publications made accessible in the public portal are retained by the authors and/or other copyright owners and it is a condition of accessing publications that users recognise and abide by the legal requirements associated with these

- Users may download and print one copy of any publication from the public portal for the purpose of private study or research.

- You may not further distribute the material or use it for any profit-making activity or commercial gain

If the publication is distributed under the terms of Article $25 \mathrm{fa}$ of the Dutch Copyright Act, indicated by the "Taverne" license above, 


\section{MICROBIOTA, OBESITY AND INSULIN RESISTANCE}

\section{Unraveling the impact of the microbiome on metabolic health in humans}


The studies presented in this thesis were performed within the framework of $\mathrm{TI}$ food and Nutrition and NUTRIM School of Nutrition and Translational Research in Metabolism. NUTRIM participates in the Graduate School VLAG (Food Technology, Agrobiotechnology, Nutrition and Health Sciences), accredited by the Royal Netherlands Academy of Arts and Sciences.
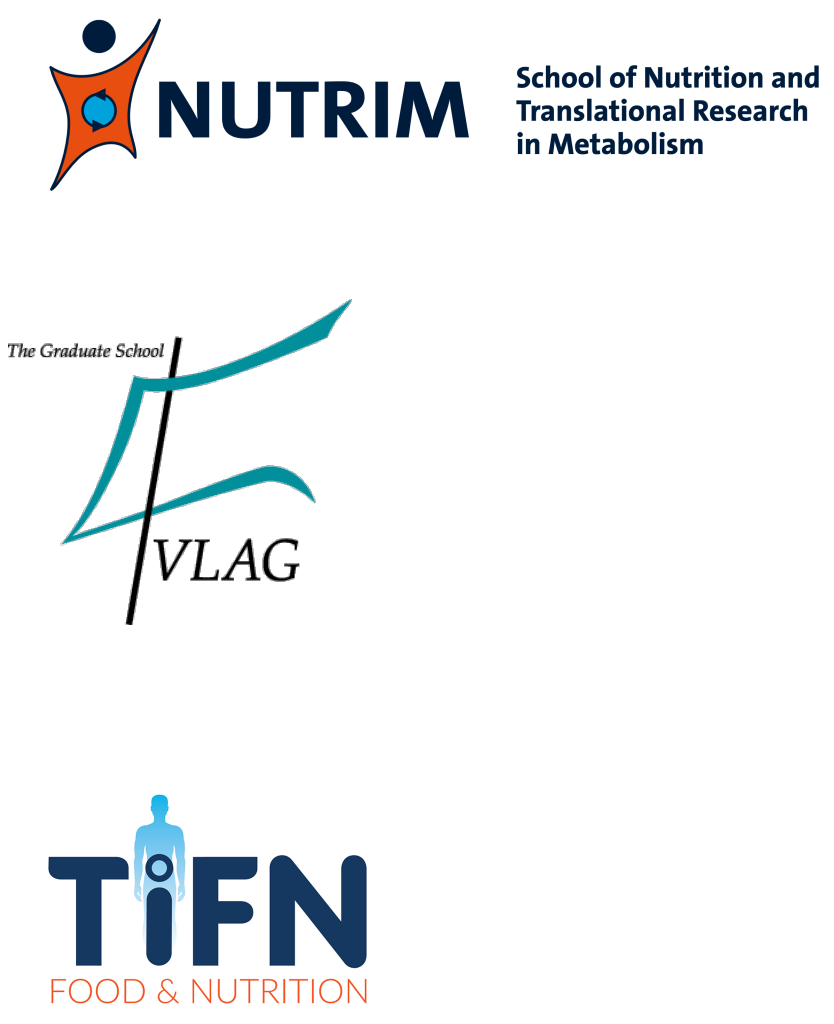

Financial support by the Netherlands association for the Study of Obesity (NASO) for the publication of this thesis is gratefully acknowledged.

(c) Dorien Reijnders, Maastricht 2016

Cover Design: StudioMik, Jack Reijnders

Layout: Dorien Reijnders

Printed by: proefschriftmaken.nl, uitgeverij BOXpress

ISBN: 978-94-6295-531 


\section{MICROBIOTA, OBESITY AND INSULIN RESISTANCE}

\section{Unraveling the impact of the microbiome on metabolic health in humans}

PROEFSCHRIFT

ter verkrijging van de graad van doctor aan de Universiteit Maastricht, op gezag van de Rector Magnificus, Prof. dr. mr. R.M. Letschert volgens het besluit van het College van Decanen, in het openbaar te verdedigen op woensdag 21 september 2016 om 14 .00 uur

door

Dorien Reijnders 


\section{Promotor}

Prof. dr. E.E. Blaak

\section{Copromotor}

Dr. G.H. Goossens

\section{Beoordelingscommissie}

Voorzitter: Prof. dr. M.A. van Baak, Maastricht Universitair Medisch Centrum

Prof. dr. A.A. Masclee, M.D., Maastricht Universitair Medisch Centrum

Prof. dr. A. Marette, Laval University, Quebec, Canada

Dr. J. Penders, Maastricht Universitair Medisch Centrum

Prof. dr. J.A.P. Willems van Dijk, Leids Universitair Medisch Centrum 
Voor Opa 

PREFACE 

'All diseases begin in the gut'. More than 2000 years after this quote from Hippocrates, we have been starting to realize how right he was. Research in the last decade demonstrated the importance of the numerous microbial gut inhabitants in health and disease. The symbiotic relationship between microbes and the human host was first described in 1683 by the pioneer in microbiology, Antoni van Leeuwenhoek. In that year, he wrote a letter to the Royal Society of London, describing "very little animalcules, very prettily a-moving," which he had seen under a microscope in plaque scraped from his teeth. In the following century, The Germ Theory of Disease was developed, which stated that many diseases are caused by the presence and actions of specific micro-organisms within the body. The more formal experiments of Louis Pasteur in the 1860s, followed by Robert Koch in the consecutive decades, provided the scientific proof for the Germ Theory.

Already in 1907, Nobel prize winner llya llitch Metchnikov, described in his book, "Essais optimistes; la longevite dans la serie animale" (The prolongation of life; optimistic studies), the numberless microbes in the large intestine, the colonization of the intestine shortly after birth, and microbial variations depending on food intake. He was also the first to describe the concept of beneficial microbes. Metchnikov worked at the Pasteur Institute, where he and Dr. Cohendy discovered that Lactobacillus bulgaricus in yoghurt and sour milk could reside in the human intestinal microbiota and would prevent against 'intestinal putrification'. They linked the lactobaccilli to longevity based on the observation of high numbers of cenetarians in Bulgarian peasant populations, who consumed large amounts of fermented milk.

For more than three centuries after van Leeuwenhoek's observation, the trillions of synbiotic microbes, remained largely unstudied. Because of the anaerobic properties of most intestinal bacteria, it was difficult to extract and culture them in a laboratory. Or, as Metchnikov 100 years ago wrote: 'unfortunately, our actual knowledge of the intestinal flora is still very imperfect because of the impossibility of finding artificial media in which it could be grown.' The development of 16 S RNA gene-based approaches in the late $20^{\text {th }}$ century finally provided the possibility to obtain a complete identification of bacteria in the human gastrointestinal tract. Since these techniques are yet widely available, research about the role of these bacteria has increased dramatically in the last 10 years. And this is just the beginning... 

Chapter 1 General Introduction

Chapter 2 Effects of Gut Microbiota Manipulation by Antibiotics on Host Metabolism in Obese Humans: a Randomized DoubleBlind Placebo-Controlled Trial

Chapter 3 Antibiotic treatment does not affect skeletal muscle 87 substrate metabolism in obese men: a randomized, doubleblind, placebo-controlled trial

Chapter 4 Gut microbiota manipulation and lipolysis in human adipose 99 tissue

Chapter 5 Microbiota composition is not correlated to insulin 117 sensitivity, but represents distinct alternative dysbiotic states in two independent cohorts of overweight and obese males

Chapter 6 General Discussion 133

Addendum Valorization 153 Summary 161

Samenvatting 165

Curriculum Vitae 169 List of Publications 171

Dankwoord 175 



\section{CHAPTER 1}

General Introduction 


\section{Obesity}

Over the last decades, the prevalence of obesity has risen to epidemic proportions, and affects all ages and socioeconomic groups in the developed world ${ }^{1,2}$. Yet, also in many low- and middle-income countries, obesity and its metabolic consequences such as type 2 diabetes mellitus ( $\left.\mathrm{T}_{2} \mathrm{DM}\right)^{3}$, cardiovascular disease, mental disorders ${ }^{4}$ and several types of cancer $^{5}$, are contributing to increased morbidity and mortality rates. The last update on the global agestandardized prevalence of overweight and obesity showed that in $2014,13.1 \%$ of men and $19.9 \%$ of all women were overweight or obese. If post-2000 trends continue, by 2025 , global obesity prevalence will reach $18 \%$ in men and surpass $21 \%$ in women. Severe obesity (body mass index $>40 \mathrm{~kg} / \mathrm{m}^{2}$ ) will surpass $6 \%$ in men and $9 \%$ in women in 2025 . These numbers indicate that obesity-related (cardiometabolic) complications will increase, accompanied by a large social and financial burden for society ${ }^{6}$.

The etiology of obesity involves the dysbalance between energy intake, energy expenditure and energy excretion, which are dependent on the interaction between environment, genetic background and other biological factors ${ }^{7}$. Moreover, the obese insulin resistant state is characterized by adipocyte hypertrophy and disturbances in adipose tissue function due to an increased adipose tissue mass ${ }^{8}$. Impairments in the adipose tissue lipid buffering capacity in obese individuals contribute to lipid overflow and deposition of fat in ectopic organs such as the liver and skeletal muscle ${ }^{9}$, in particular since the insulin resistant muscle is accompanied by an impaired capacity to increase fat oxidation upon an increased fatty acid supply ${ }^{10,11}$. The ectopic fat deposition is associated with insulin resistance ${ }^{12}$, which in concert with progressive $\beta$-cell failure results in an increased blood glucose concentration in the non-diabetic range, classified as impaired fasting glucose (IFG) or impaired glucose tolerance (IGT). IFG (fasting glucose $>5.6 \mathrm{mmol} / \mathrm{l}$ ) and IGT (2h oral glucose tolerance test (OGTT)-derived glucose concentration $>7.8 \mathrm{mmol} / \mathrm{l}$ ) are intermediate states of glucose intolerance, preceding the development of T2DM. According to the WHO Global Report on diabetes 2016, the total burden of deaths from high blood glucose has been estimated to amount 3.7 million. This number includes 1.5 million diabetes deaths, and an additional 2.2 million deaths from cardiovascular diseases, chronic kidney disease, and tuberculosis related to higher-than-optimal blood glucose $e^{13}$. 


\section{Insulin resistance}

The obesity-related ectopic deposition of lipids may reduce insulin-stimulated translocation of glucose transporters to the cell membranes, thereby disrupting glucose uptake and glycogen storage, either through direct interference of bioactive lipid-intermediates with insulin signaling or through a stimulation of inflammatory pathways ${ }^{3,11,14-16}$. Although both isolated IFG and isolated IGT are insulin resistant states, these conditions may differ in their (predominant) site of insulin resistance ${ }^{17,18}$. Subjects with isolated IFG predominantly have hepatic insulin resistance and normal or slightly lower peripheral insulin sensitivity. In contrast, individuals with isolated IGT have a normal to slightly reduced hepatic insulin sensitivity and moderate to severe peripheral insulin resistance. Consequently, combined IFG and IGT is reflected by both peripheral and hepatic insulin resistance ${ }^{19}$.

\section{Interorgan cross-talk in obesity-related insulin resistance}

The balance between energy intake, energy expenditure and energy excretion is maintained by a large regulatory network between metabolic organs. There is close interaction between the adipose tissue, skeletal muscle, pancreas and the liver in the regulation of energy and substrate metabolism. As mentioned above, disturbances in lipid metabolism are involved the etiology of obesity and related insulin resistance, impaired glucose metabolism and $\beta$-cell dysfunction.

In healthy conditions, energy that is taken up from dietary fatty acids is stored as triacylglycerol in the adipose tissue. Under conditions of a chronic positive energy balance (i.e. obesity) the adipocytes become enlarged, resulting in an impaired lipid buffering of adipose tissue when the storage capacity of adipose tissue is exceeded. Furthermore, these hypertrophic adipocytes become less sensitive to the action of insulin. Insulin is the major anti-lipolytic hormone in human adipose tissue, and induces lipid uptake from the circulation through activation of lipoprotein lipase (LPL), while at the same time suppressing lipid breakdown through inhibition of hormone-sensitive lipase $(H S L)^{20}$. Together, a reduced lipid uptake in combination with increased endogenous lipolysis causes overflow of lipids into the circulation ${ }^{8,21}$. The elevated lipid supply to non-adipose tissues such as the liver, skeletal muscle and pancreas may result in ectopic fat 
storage in these tissues if lipid supply exceeds the fat oxidative capacity ${ }^{8,11}$. High intramuscular concentrations of lipids and lipid-intermediates, driven by an impaired ability to adjust fat oxidation to the increased lipid supply and altered intramuscular fatty acid handling, disrupt insulin-mediated glucose metabolism in skeletal muscle $\mathrm{e}^{14,22}$. Also in the liver, hyperlipidemia is associated with insulin resistance and glucose intolerance ${ }^{23}$. In addition, an increased lipid supply results in an elevated hepatic triacylglycerol content and very low-density lipoprotein (VLDL-TAG) output ${ }^{11,24,25}$. Together with a higher hepatic glucose production and reduced insulin clearance ${ }^{26}$, this results in increased plasma glucose and insulin concentrations, and a decreased glucose-stimulated insulin secretion by the pancreatic $\beta$-cells on the longer term ${ }^{27,28}$.

Hypertrophic adipose tissue is furthermore characterized by a pro-inflammatory phenotype, which is the result of an imbalance between the production of proinflammatory and anti-inflammatory factors. The increased production and secretion of proinflammatory cytokines such as tumor necrosis factor alpha (TNF- $\alpha$ ) and interleukin (IL)- 6 might contribute to the development of peripheral insulin resistance and disturbances in glucose homeostasis and, therefore, precede the development of T2DM.

Recent evidence also indicates that the gut, particularly the gut microbiota and its products, is part of the inter-organ crosstalk. Intriguingly, the gut microbiota has been implied in the development of insulin resistance and impaired glucose metabolism ${ }^{29}$. More specific, there is accumulating evidence that alterations in the gut microbiota composition and function affect adipose tissue, liver and skeletal muscle lipid and glucose metabolism, at least partly through altered production of bile acids (BA), incretins, inflammatory factors and short-chain

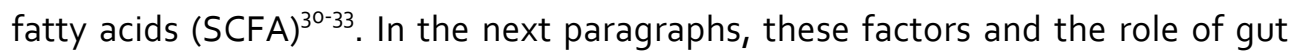
microbial imbalance (dysbiosis) in the metabolic interorgan cross-talk will be addressed in more detail.

\footnotetext{
Figure 1.1 Schematic overview of the inter-organ cross talk in obesity-induced insulin resistance. Products secreted by different tissues, including lipid species (FFA, TAG) yellow circles), proteins, hormones and inflammatory cytokines (purple stars) affect the functioning of other tissues via metabolic and endocrine cross-talk. Lipid spill-over from the adipose tissue contribute to ectopic lipid storage in the liver, skeletal muscle and pancreas, and might thereby induce insulin resistance. Also inflammatory cytokines may decrease insulin sensitivity. FFA: free fatty acids, TAG: triacylglycerol, VLDL very-low density lipoprotein, CM chylomicron.
} 


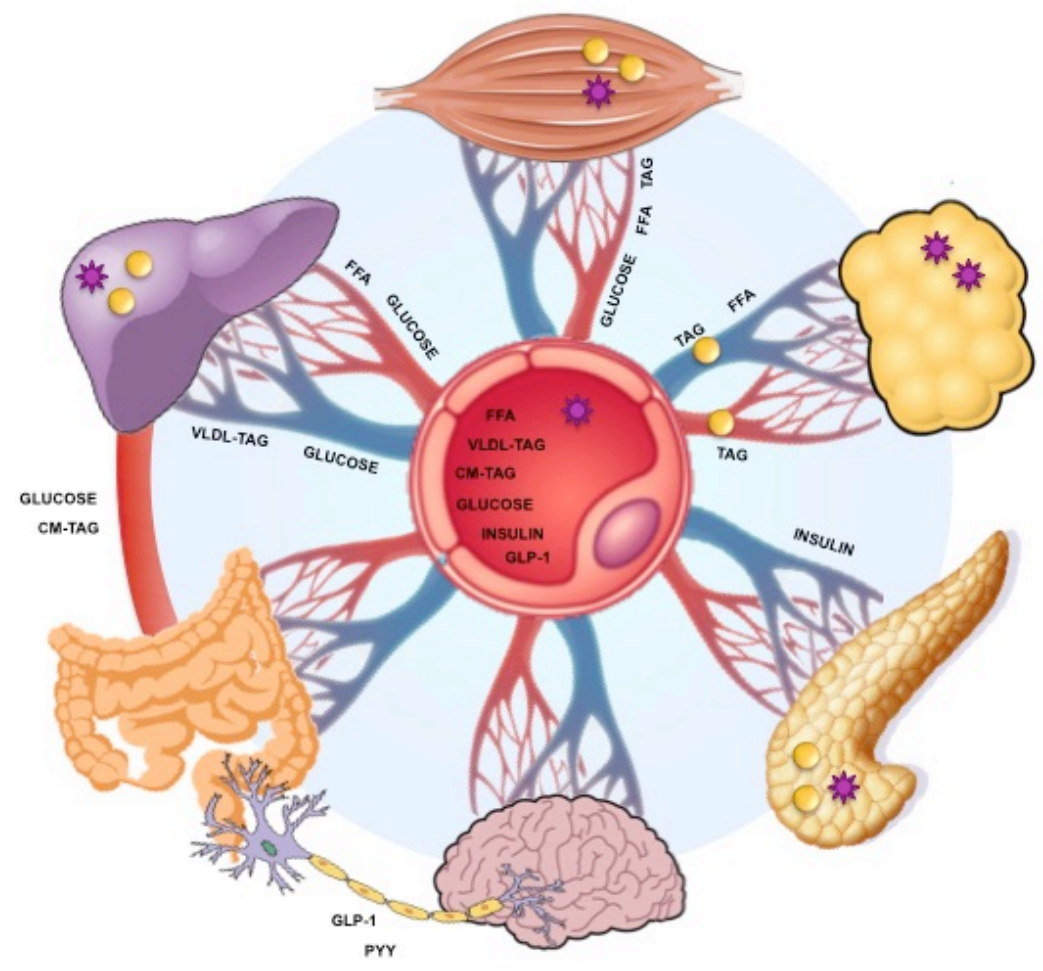

\section{The gastrointestinal tract and the microbiota within}

Anatomically, the human gastrointestinal (GI) tract has a surface of approximately $400 \mathrm{~m}^{2}$ and is composed of several compartments, each with varying metabolic functions. The digestion of food is initiated already in the mouth by chewing and the activity of amylase in saliva. After being exposed to the acidic environment $(\mathrm{pH}<2)$ in the stomach, food enters the duodenum where it is exposed to bile released from the gall bladder, and enzymes and hormones released from the pancreas. In the jejunum and ileum, food is further digested and nutrients are absorbed, whereas in the colon mainly water is resorbed. Throughout the intestine, a continuous simple columnar epithelial cell layer, which is interconnected by tight junctions and covered with a thin mucus layer, forms the physical and biochemical barrier between the host and the intestinal lumen ${ }^{34}$. 
The gastrointestinal tract is host to $>10^{14}$ microbes of all three domains of life (Bacteria, Archaea and Eukaryota) 35,36 . The microbiome is dominated by five bacterial phyla (Bacteroidetes, Firmicutes, Verrucomicrobia, Actinobacteria and Proteobacteria) and Archaea. The most relevant genera are shown in Table 1.1, which provides an overview of the bacterial taxonomy. Microbial cells outnumber human cells by 10-fold, and the collective microbial genome contains an estimated 150 times more genes than the human genome ${ }^{37,3^{8}}$. There are significant differences in concentrations and populations of microbes within the gut itself, ranging from $10^{1}$ to $10^{3}$ microbes per gram of content in the stomach and duodenum, to $10^{12}$ to $10^{14}$ microbes per gram of content in the colon ${ }^{31}$. Colonization of the $\mathrm{Gl}$ tract with bacteria is thought to begin in utero ${ }^{39}$ and is strongly influenced by the first exposure to environmental micro-organisms. Microbial profiles in infants are highly divergent and change with the introduction of solid foods to gradually reach adult profiles that show less interpersonal diversity but are still unique to a given individual ${ }^{40-42}$. In adulthood, microbial populations are typically more stable, although these can rapidly be perturbed by diet, antibiotics or states of disease, and become less diverse and more unstable again in the elderly ${ }^{27,43}$.

Depending on the type of diet, various nutrients are provided to the host and the microbiota. The microbiome encodes proteins that are not independently coded for in the human genome, including enzymes (e.g. glycoside hydrolases and bile acid hydrolases) involved in the degradation of otherwise indigestible dietary elements (e.g. resistant starch, inulin and cellulose) and bile acids. An important step in the bacterial bile acid modification is $7 \alpha / 7 \beta$-dehydroxylation, dehydrogenation or desulfation, performed by clostridia from the Firmicutesphylum. These reactions convert the most abundant primary BA in humans (cholic acid (CA) and chenodeoxycholic acid (CDCA)) into their respective secondary BA (deoxycholic acid (DCA) and litocholic acid (LCA)). Furthermore, the microbiota are involved in the biosynthesis of essential amino acids, and perform unique catabolic biotransformations, thereby producing several bioactive metabolites. Although SCFA are mainly produced from indigestible carboydrates, microbiota-dependent amino acid metabolism also plays a role in the production of SCFA. More specific, propionate and butyrate are the predominant end products of the bacterial catabolism of threonine and lysine, 
respectively ${ }^{44}$. Finally, microbiota are involved in the metabolism of xenobiotics ${ }^{45}$ and the biosynthesis of certain vitamins ${ }^{46}$.

\section{The gastrointestinal microbiota}

\subsection{Factors shaping the gut microbiota composition}

Studies in monozygotic and dizygotic twins showed that monozygotic twins have more highly correlated microbiotas, indicating a genetic component in a person's core gut microbiota composition ${ }^{47}$. During pregnancy, the developing fetal gut is 'primed' by the maternal gut microbiota and intestinal permeability, particularly towards the later stages of gestation ${ }^{41,48}$. A major determinant of the microbiota composition of newborns is the mode of delivery. Vaginally-delivered infants harbor bacterial communities resembling those of the maternal vagina, whereas gut microbiota of Caesarian section-delivered infants are enriched in maternal skin microbiota 49,50 . Following birth, the microbiota diversity develops throughout infancy and childhood, and reaches an adult-like stable composition at 2-year-old age ${ }^{51}$. Microbiota development is highly dependent on the nutritional regime (e.g. breast feeding versus formula milk ${ }^{52}$ ). Other factors such as incidental environmental exposures to antibiotics ${ }^{53}$, country of birth ${ }^{54}$ and type and duration of infection ${ }^{55}$ play a major role in the distinctive characteristics of the microbial community in the infant's first year of life. Importantly, manipulating the dynamic gut microbiota of infants by the use of antibiotics may pose an increased risk of being overweight in later childhood ${ }^{56}$.

\subsection{The human microbiota composition}

Although there is a high interindividual variability in microbial composition, the two most abundant of the 5 dominating phyla in the human gut are the Firmicutes and Bacteroidetes, comprising $>90 \%$ of all bacteria ${ }^{57}$.

The Gram-positive Firmicutes phylum is, among others, represented by the genera Ruminiococcus, Clostridium and Lactobacillus, which all comprise butyrateproducing bacterial species such as Faecalibacterium prausnitzii, Roseburia intestinalis and Lactobaccilus plantarum ${ }^{58,59}$. Lactobaccilli and clostridia are also involved in the modification of BA. A low abundance of butyrate-producing species has previously been associated with T2DM in humans ${ }^{60,61}$.

The Gram-negative Bacteroidetes phylum is represented by Bacteroides and 
Prevotella, and is able to degrade complex glycans. In the Actinobacteria-phylum, the most important commensal bacteria are from the genus Bifidobacterium, which provides the well-known probiotic species Bifidobacterium bifidum. Bifidobacteria are involved in the production of lactate and the SCFA acetate, and may thereby affect gastrointestinal immunity ${ }^{62,63}$. Proteobacteria that colonize the intestine include commensal, pathogenic and opportunistic species, such as Salmonella, Helicobacter spp., and Escherichia coli. A high abundance of Proteobacteria has been related to inflammation in rodents ${ }^{64}$. Microbes responsible for mucosal degradation are included in the phylum Verrucomicrobia, in which for example Akkermansia is classified. The Archaea domain is primarily represented by the methanogen Methanobrevibacter smithii, which is involved in the removal of bacterial end products of fermentation, thereby affecting the specificity and efficiency of bacterial digestion of dietary polysaccharides ${ }^{65}$. 


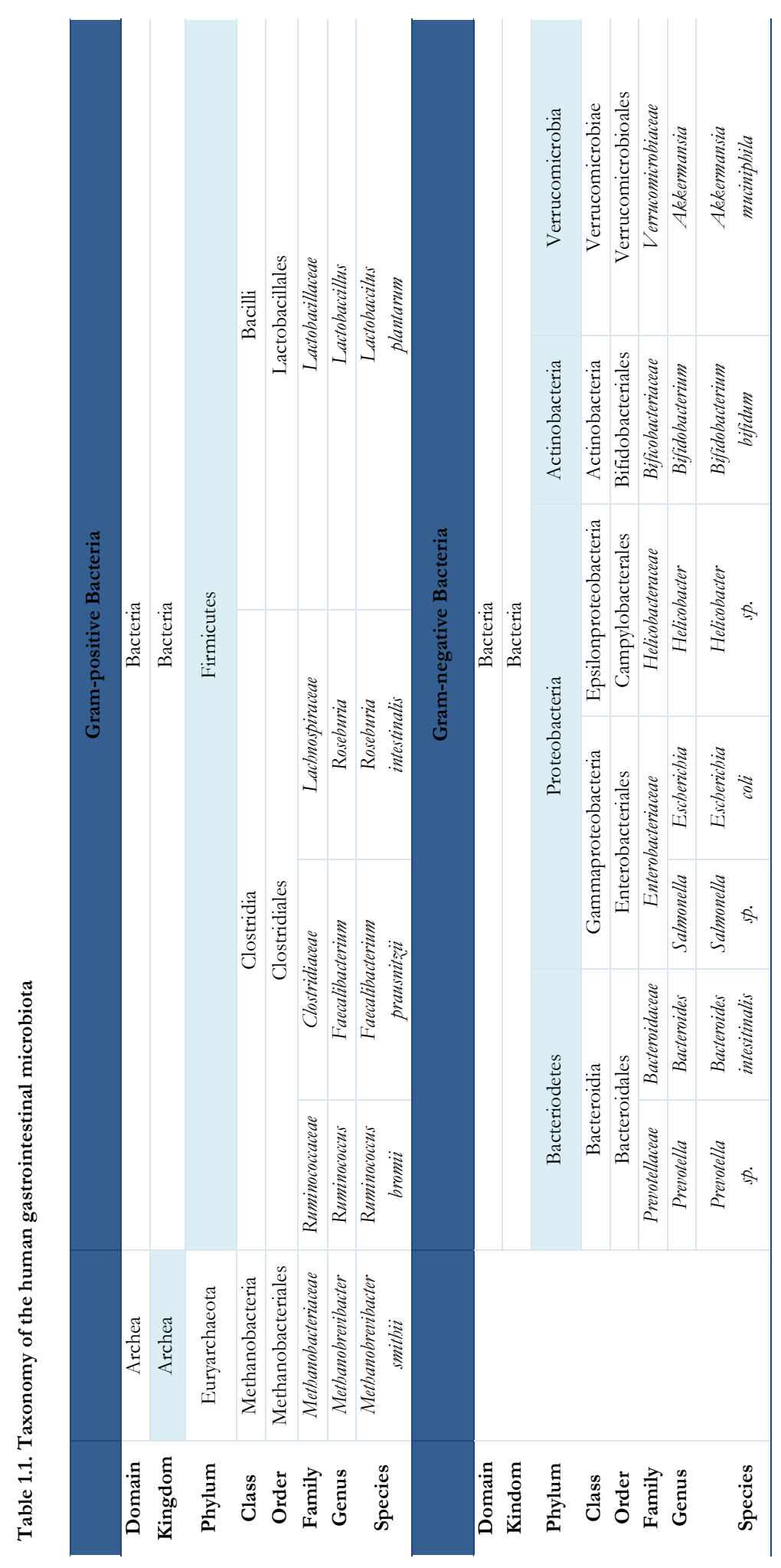




\section{Gut microbiota dysbiosis, obesity and insulin resistance}

\subsection{The discovery of an obesity-associated microbiome}

The first studies linking the gut microbiota to obesity were performed in germfree mouse models. Bäckhed et al. ${ }^{66}$ showed that 8- to 10-week-old conventionally-raised mice had $42 \%$ more total body fat than mice raised in the absence of microorganisms, even though germ-free mice consumed $29 \%$ more chow $(57 \%$ carbohydrates, $5 \%$ fat). Furthermore, when germ-free mice were colonized with the microbiota of adult conventionally-raised mice, these animals gained significantly more weight than their germ-free counterparts when fed a Western $\operatorname{diet}^{30}$. In these studies, it was hypothesized that the microbiota stimulates hepatic lipogenesis through increased monosaccharide uptake from microbial fermentation of indigestible carbohydrates, and promotes LPLdirected fat storage into adipocytes through transcriptional suppression of intestinal angiopoietin-like $4\left(\right.$ ANGPTL4) $^{30,66}$.

Studies using genetically obese mice $(o b / o b)$ indicated that these animals developed a distinct microbiome as compared to lean mice, with an increased capacity to harvest energy from the diet. This was further illustrated by showing that transferring the obese microbiota into germ-free lean mice resulted in a significantly greater increase in total body fat than colonization with a 'lean microbiota ${ }^{\prime 67}$. Ley et al. ${ }^{68}$ demonstrated that the cecal microbiota of these obese animals had a $50 \%$ reduction in Bacteroidetes and a significantly greater proportion of Firmicutes, relative to lean mice. An analogous increase in the Firmicutes/Bacteroidetes-ratio was found in the distal microbiota in obese humans ${ }^{69}$. Moreover, the dominant Firmicutes-phylum decreased after caloric restriction and Roux-en-Y gastric bypass ${ }^{70}$. Importantly, however, not all studies confirmed this correlation between obesity and the Firmicutes/Bacteroidetesratio in humans $s^{71,72}$. In a larger follow-up study in obese and lean twins, Turnbaugh et al. ${ }^{73}$ reported that the fecal microbiome of obese subjects had significantly lower proportions of Bacteroidetes and higher levels of Actinobacteria compared to lean individuals, while there was no change in the proportion of Firmicutes. Other studies found that the abundance of bifidobacteria and M. smithii ${ }^{74}$ and Akkermansia muciniphila ${ }^{75}$ was decreased in obesity, whereas Lactobacillus reuteri was increased ${ }^{74}$. Taken together, these 
data underscore that different gut microbial populations may have different effects on host adiposity, both in rodents and humans.

\subsection{The discovery of an insulin resistant and diabetes-associated microbiome}

In addition to a potential role of gut microbes in the development of obesity, alterations of gut microbial populations have been linked to altered glucose metabolism, changes in insulin sensitivity and the development of $\mathrm{T}_{2} \mathrm{DM}^{76}$. In the first study of Bäckhed et al. ${ }^{66}$, conventionalization of germ-free mice significantly increased fasting glucose and insulin levels and induced insulin resistance. Later, Larsen et al. ${ }^{61}$ demonstrated that T2DM is associated with gut compositional changes mostly apparent at phylum and class levels. The relative abundance of Firmicutes was significantly lower, while the proportion of Bacteroidetes and Proteobacteria was higher in patients with T2DM compared to healthy controls. Karlsson et al. ${ }^{77}$ identified lower abundances of Clostridium species, similar to Larsen et al. ${ }^{61}$, as well as higher abundances in four Lactobacillus species in T2DM. Lactobacillus species correlated positively, whereas Clostridium species were inversely associated with fasting glucose and HbA1c. In addition, Remely et $a .^{78}$ showed an increase in Akkermansia, Enterobacteria, and Archeae in T2DM patients. Furthermore, fecal levels of Bifdobacterium and Clostridium coccoides were found to be inversely related to fasting insulin and HOMA index in women ${ }^{79}$.

Another large metagenome-wide association study indicated that T2DM patients had only a moderate degree of gut bacterial dysbiosis ${ }^{60}$. However, functional annotation analyses did indicate a decline in butyrate-producing bacteria and an increase in opportunistic pathogens. Importantly, not only patients with T2DM but also prediabetic subjects showed a lower abundance of the butyrateproducer Faecalibacterium prausnitzii and a higher abundance of Akkermansia than glucose tolerant subjects ${ }^{80}$.

Taken together, rodent studies indicated a link between gut bacteria, insulin resistance and glucose homeostasis, which has been confirmed in human observational studies. Additional intervention studies should deepen our insight in the exact role of the microbiota composition and its functionality in human metabolism. 


\section{Gut microbiota-host interactions in obesity and insulin resistance}

The dysbiosis of the gut microbiota as found in obesity, insulin resistance and T2DM provides the host with different concentrations of microbiota-derived metabolites as compared to lean, healthy individuals. As described above, the microbiota is involved in many metabolic processes in the gut, such as fermentation of complex carbohydrates and dehydroxylation of BA. In vitro, ex vivo and in vivo studies have suggested multiple mechanisms by which these and other microbial products can interfere with the gut-brain axis and the cross-talk between the adipose tissue, skeletal muscle, liver and pancreas. The following paragraphs provide an overview of important gut-derived factors, their receptors and signaling pathways, which might provide the link between gut microbiota and host physiology, thereby playing a role in the etiology of obesity and T2DM.

\subsection{Microbiota-related effects on energy regulation}

The presence of gut bacteria has been found to influence energy balance, since germ-free mice have significantly lower body weight and body fat percentage, despite a higher caloric intake than conventionalized mice ${ }^{66}$. The gut microbiota might affect both sides of the energy balance, as a factor that influences energy intake and energy harvesting, as well as energy expenditure ${ }^{30,32}$.

\section{Energy intake and satiety}

First, the gut microbiota produces SCFA, which through a G-protein coupled receptor (GPR) 41 dependent mechanism might modulate the secretion of incretin hormones like glucagon-like peptide-1 (GLP-1) and the expression of Peptide-YY (PYY) ${ }^{81}$. GLP-1 and PYY are satiety signals that can drive insulin secretion $^{82}$ and regulate food intake and blood glucose via complex overlapping endocrine and neuronal pathways ${ }^{83}$. Indeed, in humans with obesity, inulin propionate-ester intake versus inulin alone for 24 weeks prevented body weight gain through effects on appetite regulation ${ }^{84}$. Thus, by modulating SCFA production and subsequent satiety signals, the gut microbiota composition may affect energy intake and, therefore, energy balance. 


\section{Energy harvesting}

An additional role for microbiota in energy balance is the capacity to increase energy harvest from the diet, mainly through fermentation of indigestible carbohydrates. The conversion of polysaccharides to acetate, butyrate or propionate accounts for approximately $5-10 \%$ of the daily energy uptake ${ }^{85,86}$, but thus far evidence in humans is scarce. Although the concept of altered energy harvest as a mechanism that may contribute to differences in body weight or body composition is used frequently throughout the scientific literature ${ }^{30,66}$, the significance of (altered) energy harvest from indigestible carbohydrates in humans is currently largely unknown.

\section{Energy expenditure}

Accompanied by an increased provision of energy substrates, the gut microbiota may also affect processes that favor energy storage over energy expenditure. Supportive for the role of microbiota-derived BA in energy regulation is a study in FXR-deficient mice, which had a reduced adipose tissue mass ${ }^{87,88}$ possibly mediated via pronounced activation of the Wnt/B-catenin signaling pathway, which inhibits adipocyte differentiation and PPARY function. Furthermore, the gut microbiota might enhance LPL-directed storage of (de novo) triglycerides in adipocytes. This was suggested upon the finding that conventionalization of germ-free mice promoted adipocyte hypertrophy by suppressing the intestinal expression of the circulating LPL inhibitor ANGPTL4 ${ }^{30,66 .}$

Lastly, the intestinal microbiota has been found to be involved in the expression of the fuel sensor adenosine monophosphate-activated protein kinase (AMPK) in the liver and skeletal muscle, which plays a key role in fatty acid $\beta$-oxidation and energy expenditure ${ }^{89}$. Germ-free mice were resistant to a high-fat diet and showed a $40 \%$ increase of phosphorylated-AMPK in skeletal muscle as compared to conventionally-raised mice, which was reversible upon microbiota transplantation in these mice ${ }^{30}$.

In summary, the gut microbiota has been suggested to play a role in energy harvesting, energy intake and energy expenditure in rodents, thereby affecting energy balance and the development of obesity. However, evidence in humans in largely lacking. 


\subsection{Microbiota and systemic low-grade inflammation}

The microbiota residing in the colon plays an important role in the host immune system via interaction with the intestinal epithelium ${ }^{90}$. Peptide-glycans, such as the Gram-negative bacterial cell wall component lipopolysaccharides (LPS), can be recognized by stromal and epithelial cells, through pattern recognition receptors such as Toll-like receptor $\left.4\left(T_{L}\right)_{4}\right)^{91-93}$. Subsequent activation of proinflammatory kinases and transcription factors (e.g. nuclear factor kappa $B$, $N F k B$ ), causes an increased expression of inflammatory factors such as TNF- $\alpha$, monocyte chemotactic protein (MCP)-1 and IL-6, which have been associated with the induction of insulin resistance in various tissues in rodents ${ }^{94}$.

Additionally, LPS can reach the systemic circulation via uptake in chylomicrons or intestinal translocation. Indeed, high circulating levels of LPS have been associated with increased systemic inflammation ${ }^{91,95}$ and lower intestinal integrity in subjects with obesity, the metabolic syndrome and $\mathrm{T}_{2} \mathrm{DM}^{96}$. Furthermore, human studies demonstrated that circulating levels of bacterial DNA, mainly derived from Proteobacteria, were enhanced in prediabetes, indicating a role for gut bacteria in metabolic inflammation ${ }^{97}$.

Microbiota-produced SCFA might enhance the intestinal barrier and decrease gut permeability, e.g. by modulating the expression of tight junction protein and mucins, thereby decreasing LPS translocation and systemic inflammation ${ }^{76,98}$. Indeed, butyrate has been found to regulate the colonic size and function of the anti-inflammatory regulatory T-cell network ${ }^{99-101}$ and to decrease the expression of pro-inflammatory cytokines. Also the expression of the SCFA receptor GPR43 in neutrophils, macrophages and monocytes ${ }^{102,103}$ suggests that microbiotadependent products such as SCFA may play a role in peripheral inflammation ${ }^{99}$. In fact, acetate and butyrate decreased LPS-stimulated release of TNF- $\alpha$ from human neutrophils in vitro $^{104}$ and inhibited NFkB-expression in murine macrophage cell lines ${ }^{105}$.

Also the BA receptors $\mathrm{FXR}$ and $\mathrm{G}$-protein coupled receptor $\mathrm{TGR}_{5}$ are highly expressed in the Gl-tract, monocytes and macrophages as well as other immune cell types ${ }^{106,107}$. Therefore, BA are thought to be involved in the regulation of a normal Gl inflammatory tone, the prevention of pathogen invasions and maintaining cell integrity ${ }^{33}$. Deconjugated and secondary BA within the GI tract counterregulate activities of innate immune cells, leading to the inhibition of inflammatory cytokine production in macrophages and monocytes, involving a 
FXR-mediated inhibition of NFkB activity ${ }^{93,108,109}$. Abnormalities in BA metabolism have been linked to gut microbiota dysbiosis in the metabolic syndrome and inflammatory disorders such as inflammatory bowel disease or irritable bowel syndrome ${ }^{110}$, but also in nonalcoholic steatohepatitis ${ }^{92}$.

To summarize, the interactions between gut microbiota, epithelial cells and the immune system are the main framework underlying a possible altered intestinal permeability and low-grade inflammation in obesity, and might therefore also play an important role in insulin resistance.

\subsection{Microbiota, glucose homeostasis and insulin sensitivity}

As briefly described above, the microbiota composition may be related to insulin resistance ${ }^{111}$. In fact, transplantation of the microbiota from lean donors to recipients with the metabolic syndrome slightly improved insulin sensitivity in a subset of the study participants ${ }^{112}$. It has previously been found that butyrateproducing species are often decreased in T2DM patients ${ }^{60,61,77}$, suggesting a role for SCFA in the pathogenesis of insulin resistance. In line, the improved insulin sensitivity after fecal transplantation was accompanied with higher levels of butyrate-producing bacteria. Depletion of certain butyrate-producing strains by the antibiotic vancomycin, on the other hand, was found to slightly but significantly decrease insulin sensitivity ${ }^{113}$. Additionally, inflammation-related Proteobacteria increased after vancomycin treatment, suggesting a role for inflammatory cytokines in insulin signaling. In addition, insulin sensitivity might be influenced by the metabolism of BA in the gut. The antibiotic-induced alteration of the gut microbiota of obese subjects slightly decreased peripheral insulin sensitivity, with a concomitant decrease of fecal secondary BA and a simultaneous increase in postprandial primary BA. In contrast, another study with short-term antibiotic exposure in healthy volunteers did not find effects on glucose tolerance ${ }^{114}$, whereas broad-spectrum antibiotics in mice improved rather than impaired fasting glucose levels and glucose tolerance ${ }^{115}$.

As an alternative approach, animal and human studies demonstrated that microbiota manipulations by probiotics improved glucose tolerance ${ }^{116}$. For example, Akkermansia-administration enhanced glucose tolerance in high-fat diet-fed mice ${ }^{117}$. A recent meta-regression analysis identified that probiotics can significanlty reduce glucose, $\mathrm{HbA1c}$, insulin and HOMA-IR in participants with T2DM, but not in participants with other risk factors such as hypertension, hyperlipidaemia or obesity ${ }^{118}$. 
To conclude, although rodent studies have suggested several mechanisms that relate gut microbiota composition to insulin resistance and $T_{2} \mathrm{DM}$, human data is scarce and inconclusive. Therefore, it is important to determine which microbiota-related mechanisms regulate insulin resistance, how different organs are affected and which mechanisms are disturbed in the interorgan crosstalk in humans.

\subsection{Microbiota-related effects on adipose tissue metabolism}

\section{Adipose tissue inflammation}

The low-grade inflammatory state in obesity and T2DM is characterized by increased production and secretion of proinflammatory cytokines and adipokines by adipocytes $s^{8,11,94}$. The M1-polarization of adipose tissue macrophages increases the production of TNF- $\alpha$ and IL-6, which can directly inhibit insulin action in target cells (e.g. hepatocytes, myocytes and adipocytes) via autocrine, paracrine and endocrine mechanisms ${ }^{94}$. Moreover, the expression of SCFA and BA receptors on macrophages and adipocytes indicates a role for a gut-adipose tissue interaction in the adipose tissue inflammatory phenotype ${ }^{119}$. Acetate, propionate and butyrate might all prevent chronic low-grade inflammation by upregulating colonic anti-inflammatory $T_{\text {REG }}$ cells dependent on GPR 43, thereby decreasing metabolic endotoxemia and reducing production of proinflammatory adipocytokines and chemokines in mice. Overall, in addition to interacting with systemic inflammation, the gut microbiota composition might be involved in adipose tissue inflammation by effects on AT macrophages and monocytes. However, most data is derived from animal studies or cell models and therefore needs to be confirmed in humans.

\section{Lipolysis}

In the postprandial period, VLDL- and chylomicron-TAG are hydrolyzed in the process of extracellular lipolysis by $L P L^{120}$. The FFAs that are liberated by this process can be taken up by the adipose tissue and can be directed towards oxidation or storage, or are spilled over and remain in the circulation ${ }^{121}$. The activity of LPL is regulated by nutrients and hormones such as insulin and ANGPTL4 ${ }^{122-124}$. In obesity, insulin resistance and T2DM, the removal of TAG across adipose tissue is impaired due to a reduced insulin-mediated stimulation of $L P L$ activity ${ }^{8,125}$. A role for the gut microbiota in LPL activation has been 
suggested via the transcriptional suppression of ANGPTL4 by gut microbiota upon conventionalization in germ-free mice ${ }^{66}$.

Moreover, ANGPTL4 might also reduce intracellular lipolysis under fasting conditions and during catecholamine stimulation in murine adipocytes ${ }^{126,127}$. Intracellular lipolysis describes the hydrolysation of TAG into glycerol and FFA by adipose tissue lipase (ATGL) and hormone-sensitive lipase (HSL), and involves a complex pathway that is dependent on the regulation of lipolytic and antilipolytic hormones and molecules. Catecholamines and TNF- $\alpha$ induce lipolysis, whereas insulin is the main antilipolytic hormone ${ }^{128}$. The production of SCFA in the gut, which relates to the microbiota composition and diet, might affect adipose tissue lipolysis. Several human studies have shown that oral or rectal administration of SCFA, in particular acetate, significantly decreased plasma free fatty acid (FFA) concentrations $^{129-131}$.

Treatment of differentiated murine $3^{T} T_{3}-L_{1}$ adipocytes with acetate and propionate reduced intracellular fasting and catecholamine-mediated lipolysis via activation of GPR43, which might be explained by a decreased HSL phosphorylation $^{119}$. In addition, binding of SCFA to GPR41 on adipocytes inhibited lipolysis, and increased adipogenesis and leptin synthesis in mice ${ }^{132}$. In the absence of the BA receptor FXR (FXR knock-out mouse model) basal intracellular lipolysis was increased, leading to elevated plasma FFA levels, indicating that BA signaling might also be involved in adipose tissue biology.

In summary, although several studies have indicated mechanisms via which the gut microbiota composition might affect adipose tissue lipolysis, the exact mechanisms in human physiology remain to be elucidated.

\section{Adipogenesis}

The differentiation of pre-adipocytes into mature adipocytes is known as adipogenesis. This process is highly dependent on peroxisome proliferatoractivated receptor $\gamma$ (PPAR $\gamma$ ), which encodes a regulatory protein of adipocyte differentiation. During persistent positive energy balance, the adipose tissue is forced to expand to facilitate increased TAG storage ${ }^{8,11}$ The inability of the adipose tissue to differentiate new adipocytes and store excess lipids in mature adipocytes results in adipocyte hypertrophy. Impaired adipose tissue expandability is associated with adipose tissue dysfunction, which is characterized by decreased insulin sensitivity, increased immune cell infiltration and increased intracellular and systemic FFA flux ${ }^{8}$. It has been shown in a FXR 
knock-out model, that the BA receptor FXR controls adipocyte differentiation and function by promoting PPARY activity and inhibiting the Wnt/ $\beta$-catenin pathway ${ }^{88,133}$. In addition, a role for FXR activation has been suggested in adipogenesis and insulin signaling ${ }^{133}$, which might be due to reduced lipogenesis ${ }^{87,88}$. Moreover, a role for SCFA in adipogenesis has been found, as propionate induced GPR43 expression during adipose differentiation of ${ }_{3} \mathrm{~T}_{3}-\mathrm{L}_{1}$ cells, with an up-regulation of PPAR ${ }^{134}$.

Taken together, several studies suggest a role for BA and SCFA on adipogenesis and PPARY activity. However, the exact mechanisms by which the microbiota composition might be involved in adipogenesis, need to be further investigated in human in vivo models.

\subsection{Microbiota-related effects on liver metabolism}

\section{Hepatic inflammation}

Although the intestinal mucosa acts as an effective barrier against translocation of microbes and microbial products such as LPS and bacterial DNA from the gut to the circulation, small quantities do enter the portal vein. The liver is thereby the first filter for nutrients and microbial products that can alter glucose production and increase inflammation, steatosis, and insulin resistance. For example, the gut microbiota endotoxin LPS might set off the inflammatory cascade in the liver via binding to TLR- 4 and consequent activation of Kupffer cells $^{135}$. In addition, LPS has been found to play a role in the development of steatohepatitis in obese patients ${ }^{92}$. In line, bacterial DNA has been found in the liver $^{136}$ and NASH patients presented with a higher abundance of Proteobacteria in their intestines ${ }^{137}$. The increased inflammatory environment in the liver, together with hepatic lipid accumulation, plays a role in the development of hepatic insulin resistance.

\section{Hepatic lipid regulation}

Lipids derived from adipose tissue spillover and a high dietary fat intake increase the flux of lipids to the liver, which as a consequence enhances the production and secretion of VLDL particles, and thereby increases cardiovascular disease risk. The gut microbiota has been indicated to be involved in hepatic lipid regulation, via stimulation of hepatic triglyceride production mediated by transcription factors such as ChREBP ${ }^{66}$, and the inhibition of $\mathrm{ANGPTL}_{4}{ }^{66}$. In addition, 
microbiota-derived SCFA might play a role, as acetate and proprionate, taken up by the liver, may be used as substrates for lipogenesis and gluconeogenesis ${ }^{138}$. Furthermore, acetate has been found to induce the liver enzyme $C Y P 7 A_{1}$, responsible for $B A$ synthesis ${ }^{139}$. A study in mice showed, that the increase of the bile acid pool size by overexpression of $\mathrm{CYP}_{7} \mathrm{~A}_{1}$, protected from Western dietinduced obesity, hepatic steatosis and insulin resistance ${ }^{140}$.

Taken together, the gut microbiota could influence signaling pathways involved in energy and lipid metabolism, leading to alterations in the production of hepatic fatty acids and triglyceride storage.

\subsection{Microbiota-related effects on skeletal muscle metabolism}

\section{Insulin signaling}

The skeletal muscle is the main site responsible for insulin-stimulated glucose disposal ${ }^{141}$. In postprandial conditions, insulin stimulates the transport of the glucose transporter GLUT4 to the cell membrane, thereby facilitating glucose uptake into the muscle ${ }^{142}$. In insulin resistant obesity, intramuscular toxic lipidintermediates and inflammatory molecules inhibit the insulin signaling cascade, thereby contributing to elevated blood glucose concentrations, which in turn predispose towards $\mathrm{T}_{2} \mathrm{DM}^{13,14,15}$. As described above, $\mathrm{T}_{2} \mathrm{DM}$ and insulin resistance are associated with compositional changes of the microbiota ${ }^{60,61,75,77}$. These compositional changes might affect concentrations of endotoxin and inflammatory cytokines. In addition to the general assumption that cytokines inhibit insulin signaling, it has been found that also plasma LPS levels were negatively correlated with clamp-derived whole-body insulin sensitivity ${ }^{143,144}$. A recent study has shown that $B A$ might affect skeletal muscle insulin sensitivity in mice, since the intestinal expression of FXR was required for the development of insulin resistance and obesity in response to a high-fat diet ${ }^{145}$. Moreover, BA can activate TGR5 in mice, which is expressed in skeletal muscle, thereby exerting protective effects against diet-induced obesity and insulin resistance ${ }^{146}$.

\section{Substrate metabolism}

Impairments in fasting and postprandial fatty acid uptake, storage and oxidation may contribute to intramuscular accumulation of lipid-intermediates like diacylglycerol and ceramides ${ }^{22,147}$. It has been indicated that the metabolic profile of the insulin resistant muscle is more directed towards fat storage rather than 
oxdation $^{148,149}$. The lipid content in the muscle reflects a net balance between rates of fatty acid supply, uptake and mitochondrial fat oxidation ${ }^{11}$. As described above, the intestinal microbiota has been found to be related to the expression of AMPK in the muscle, which plays a key role in fatty acid $\beta$-oxidation ${ }^{30}$. Furthermore, SCFA may affect muscle insulin sensitivity and glucose metabolism through intestinal GLP-1 secretion. It has been demonstrated in rats that GLP-1 might modulate muscle microvascular function, thereby altering substrate supply and uptake in skeletal muscle ${ }^{150}$. SCFA-stimulated secretion of PYY might also improve insulin-mediated glucose uptake in skeletal muscle and increase wholebody fat oxidation, as shown in animal studies ${ }^{151,152}$. Finally, SCFA may alter peripheral substrate metabolism since their receptors, GPR 41 and 43 , are found to be expressed in skeletal muscle ${ }^{153,154}$.

Taken together, several lines of evidence suggest that the gut microbiotaskeletal muscle crosstalk is involved in substrate metabolism and insulin sensitivity ${ }^{32,155}$. However, human studies that provide evidence for this have not been perfomed yet.

\subsection{Microbiota-related effects on insulin secretion}

Gut-derived factors might have an effect on insulin secretion by pancreatic $\beta$ cells. GLP-1 is the primary incretin hormone secreted from L-cells in the intestine, which may affect pancreatic $\beta$-cells by increasing insulin secretion ${ }^{156}$. At the same time, GLP-1 may decrease glucagon release from $\alpha$-cells ${ }^{157}$. Indeed, elevated GLP-1 levels were correlated with improved glucose homeostasis in patients after bariatric surgery ${ }^{158}$. As discussed earlier, SCFA have been indicated to modulate the secretion of incretin hormones through a GPR41-dependent mechanism ${ }^{81}$. Moreover, a recent study has demonstrated a regulatory pathway for the SCFAGPR43 axis in the modulation of $\beta$-cell function ${ }^{159}$. In addition, BA might be involved in the regulation of insulin release. The expression of BA receptor FXR has recently been reported to play a role in the pancreas, where it regulates glucose-induced insulin secretion ${ }^{160,161}$ and protects against lipotoxicity ${ }^{160}$. Taken together, these findings highlight a novel relationship between the gut microbiota composition and glucose homeostasis. However, human studies that have investigated the effect of the gut microbiota on $\beta$-cell function and insulin secretion are lacking. 


\section{How to target the adult gut microbiota composition?}

The resilient microbiota of adults can be subjected to minor and major disturbances such as infection, exposure to antibiotics and major dietary shifts ${ }^{162}$. Diet can specifically influence the microbiota via the diet composition and total energy content of the diet, or indirect via regulation of the gastrointestinal $\mathrm{pH}$ and transit time ${ }^{163}$. Long-term consumption of hunther-gatherer, plant-based diets have been associated with greater microbiome diversity ${ }^{43}$, increased abundance of Prevotella and higher levels of SCFA production. The animal-based, high-fat Western diet, on the other hand, is associated with the Bacteroides enterotype ${ }^{57}$. The addition of prebiotic fibers to a diet, such as resistant starch, has been found to reduce mesenteric (visceral) fat accumulation, enhance insulin sensitivity, improve blood glucose levels and lipid metabolism ${ }^{164}$. Probiotics, consisting of one or more species of live bacteria such as lactobacilli and bifidobacteria, have been found not only to affect the intestinal flora directly but are also able to modulate immunological parameters and intestinal permeability, produce regulatory metabolites and improve glucose homeostasis ${ }^{16,165-167}$. The strong influence of dietary habits is underscored by the pronounced distinction of the gut microbial profiles of U.S. vs. Malawian and Amerindian populations across all ages ${ }^{43}$. In addition, recent evidence has indicated that even visits of short duration to other geographic locations can influence the gut microbial composition $^{168}$. Lastly, short-term interventions with different diets varying in macronutrient composition have demonstrated that the gut microbiome can rapidly respond to altered diet, reflecting the diversity of human dietary lifestyles ${ }^{169}$.

A more robust way to manipulate the microbiota composition is by administration of antibiotics, which can be selectively administered to target a specific pathogenic population. Antibiotic treatment typically induces a decrease in the diversity of the microbiota ${ }^{170}$. Antibiotics shift the composition of the microbiota in different ways, depending on their spectrum of activity, thereby specifically changing the metabolic profiles of intestinal contents and feces ${ }^{171}$. For example, models of antibiotic-treated mice show reduced fecal BA and decreased BA diversity, as a result of reduced microbial BA metabolism ${ }^{33,172}$. Another common feature of the metabolic profiles of antibiotic-treated mice and humans is a reduced production of SCFA ${ }^{173-175}$. Moreover, long-term antibiotic exposure has been found to promote weight gain ${ }^{174,175}$ and, therefore, was among 
the first growth promoters used in agriculture ${ }^{176}$. Importantly, when animals were exposed to sub-therapeutic dosages of antibiotics early in life, the effects on growth promotion were greater than if the exposure occured later in life, indicating that early life is a metabolically vulnerable stage ${ }^{177}$. Furthermore, it has been demonstrated that the risk of developing T2DM increases with the number of antibiotic prescriptions, together with a decline of intestinal integrity ${ }^{178}$. However, mouse studies have shown that antibiotic treatment improved metabolic impairments such as insulin resistance and glucose homeostasis ${ }^{175,179,180}$.

Overall, it has been shown that antibiotic treatment can alter the microbiota composition, with consequences for the host metabolic phenotype. Therefore, antibiotic-treatment is a relative useful tool to investigate the role of the microbiota in human metabolism.

\section{Outline of this thesis}

Research performed to date has demonstrated that the gut microbiota may be related to host energy and substrate metabolism. Moreover, perturbations of microbial populations may contribute to an obesogenic phenotype, inflammation, an altered glucose metabolism, and insulin resistance. Importantly, however, mechanistic studies almost exclusively have been performed in rodent models. Therefore, these rodent studies need to be confirmed and/or extended to human clinical trials to better understand if and how specific microbial populations may contribute to the development of obesity and related metabolic and inflammatory perturbations, including the development of T2DM.

This thesis focuses on the effects of microbiota manipulation using broadspectrum and small-spectrum antibiotics in obese, insulin resistant humans with an impaired glucose metabolism, taking into account multiple mechanisms that have been proposed to contribute to metabolic impairments based on rodent studies. Chapter 2 describes the results of a double-blind, randomized placebocontrolled trial that was designed to investigate the effects of antibiotic treatment on tissue-specific insulin sensitivity, the gut microbiota composition, SCFA and BA concentrations. Moreover, this chapter also describes secondary outcomes of energy expenditure and substrate utilization, gut permeability, inflammation, adipocyte morphology and the adipose tissue transcriptome. In the same clinical trial, the effect of antibiotics on forearm blood flow and 
substrate fluxes across forearm muscle were investigated before and after consumption of a high-fat mixed-meal. Substrate fluxes of glucose, TAG, FFA and lactate across the forearm muscle were assessed by combining measurements of arterio-venous concentration differences across forearm muscle and forearm blood flow (venous occlusion plethysmography). The results of these measurements are described in Chapter 3. To obtain insight into the mechanisms that might affect lipid metabolism and insulin sensitivity in adipose tissue after antibiotic treatment, Chapter 4 describes the effects of microbiota manipulation on ex vivo lipolysis in freshly isolated abdominal subcutaneous adipocytes. In this cell model, both basal and $\beta$-adrenergically-stimulated lipolysis were measured. Moreover, the effects of antibiotic treatment on adipose tissue expression of lipolytic genes and proteins were examined in human adipose tissue.

Observational studies have indicated that the gut microbiota composition is associated with T2DM and insulin resistance. However, most studies compared the microbiome between lean and obese or T2DM subjects, without having examined host metabolic characteristics in detail. Therefore, in Chapter 5, we aimed to obtain a better insight in the association between the microbiota composition and peripheral, hepatic and adipose tissue insulin sensitivity. This chapter describes a cross-sectional analysis of two independent cohorts of obese men (Maastricht and Amsterdam), in which the relationship between gut microbiota composition and tissue-specific insulin sensitivity was investigated in detail.

Finally, the findings of the studies described in this thesis are discussed in Chapter 6, in addition to implications for future studies 


\section{References}

1. Obesity and overweight Fact sheet $\mathrm{N}^{\circ} 3112015$.

2. Hill JO. Understanding and addressing the epidemic of obesity: an energy balance perspective. Endocrine reviews 2006;27:750-61.

3. Guilherme A, Virbasius JV, Puri V, Czech MP. Adipocyte dysfunctions linking obesity to insulin resistance and type 2 diabetes. Nature reviews Molecular cell biology 2008;9:367-77.

4. Preiss K, Brennan L, Clarke D. A systematic review of variables associated with the relationship between obesity and depression. Obesity reviews : an official journal of the International Association for the Study of Obesity 2013;14:906-18.

5. Renehan AG, Zwahlen M, Egger M. Adiposity and cancer risk: new mechanistic insights from epidemiology. Nature reviews Cancer 2015;15:484-98.

6. Trends in adult body-mass index in 200 countries from 1975 to 2014: a pooled analysis of 1698 population-based measurement studies with 19\&\#xb7;2 million participants: Elsevier; 2016. Report No.: 0140-6736.

7. Alberti KG, Zimmet $P$, Shaw J, Group IDFETFC. The metabolic syndrome--a new worldwide definition. Lancet 2005;366:1059-62.

8. Goossens $\mathrm{GH}$. The role of adipose tissue dysfunction in the pathogenesis of obesity-related insulin resistance. Physiology \& behavior 2008;94:206-18.

9. McQuaid SE, Hodson L, Neville MJ, et al. Downregulation of adipose tissue fatty acid trafficking in obesity: a driver for ectopic fat deposition? Diabetes 2011;60:47-55.

10. Kelley DE, Goodpaster B, Wing RR, Simoneau JA. Skeletal muscle fatty acid metabolism in association with insulin resistance, obesity, and weight loss. The American journal of physiology 1999;277:E1130-41.

11. Stinkens $\mathrm{R}$, Goossens $\mathrm{GH}$, Jocken JW, Blaak EE. Targeting fatty acid metabolism to improve glucose metabolism. Obesity reviews : an official journal of the International Association for the Study of Obesity 2015;16:715-57.

12. Cooke AA, Connaughton RM, Lyons CL, McMorrow AM, Roche HM. Fatty acids and chronic low grade inflammation associated with obesity and the metabolic syndrome. European journal of pharmacology 2016.

13. WHO Global report on diabetes2016.

14. Samuel VT, Shulman GI. The pathogenesis of insulin resistance: integrating signaling pathways and substrate flux. The Journal of clinical investigation 2016;126:12-22.

15. Samuel VT, Shulman GI. Mechanisms for insulin resistance: common threads and missing links. Cell 2012;148:852-71. 
16. Farese RV, Standaert ML, Ishizuka T, Yu B, Arnold TP, Cooper DR. The role of diacylglycerol/protein kinase $C$ signaling in insulin-stimulated glucose transport. Transactions of the Association of American Physicians 1991;104:187-93.

17. Abdul-Ghani MA, Jenkinson CP, Richardson DK, Tripathy D, DeFronzo RA. Insulin secretion and action in subjects with impaired fasting glucose and impaired glucose tolerance: results from the Veterans Administration Genetic Epidemiology Study. Diabetes 2006;55:1430-5.

18. Qiao Q, Jousilahti P, Eriksson J, Tuomilehto J. Predictive properties of impaired glucose tolerance for cardiovascular risk are not explained by the development of overt diabetes during follow-up. Diabetes care 2003;26:2910-4.

19. Nathan DM, Davidson MB, DeFronzo RA, et al. Impaired fasting glucose and impaired glucose tolerance: implications for care. Diabetes care 2007;30:753-9.

20. Goldberg IJ, Eckel RH, Abumrad NA. Regulation of fatty acid uptake into tissues: lipoprotein lipase- and $\mathrm{CD}_{3} 6$-mediated pathways. Journal of lipid research 2009;50 Suppl:S86-9o.

21. Frayn KN. Insulin resistance, impaired postprandial lipid metabolism and abdominal obesity. A deadly triad. Medical principles and practice : international journal of the Kuwait University, Health Science Centre 2002;11 Suppl 2:31-40.

22. Ritter $\mathrm{O}$, Jelenik T, Roden M. Lipid-mediated muscle insulin resistance: different fat, different pathways? Journal of molecular medicine 2015;93:831-43.

23. Perry RJ, Samuel VT, Petersen KF, Shulman GI. The role of hepatic lipids in hepatic insulin resistance and type 2 diabetes. Nature 2014;510:84-91.

24. Bissonnette $\mathrm{S}$, Salem $\mathrm{H}$, Wassef $\mathrm{H}$, et al. Low density lipoprotein delays clearance of triglyceride-rich lipoprotein by human subcutaneous adipose tissue. Journal of lipid research 2013;54:1466-76.

25. Nielsen S, Karpe F. Determinants of VLDL-triglycerides production. Current opinion in lipidology 2012;23:321-6.

26. Wiesenthal $S R$, Sandhu $H, M c C a l l ~ R H$, et al. Free fatty acids impair hepatic insulin extraction in vivo. Diabetes 1999;48:766-74.

27. Giacca A, Xiao C, Oprescu Al, Carpentier AC, Lewis GF. Lipid-induced pancreatic beta-cell dysfunction: focus on in vivo studies. American journal of physiology Endocrinology and metabolism 2011;300:E255-62.

28. Poitout V, Amyot J, Semache M, Zarrouki B, Hagman D, Fontes G. Glucolipotoxicity of the pancreatic beta cell. Biochimica et biophysica acta 2010;1801:289-98.

29. Harris K, Kassis A, Major G, Chou CJ. Is the gut microbiota a new factor contributing to obesity and its metabolic disorders? Journal of obesity 2012;2012:879151. 
30. Backhed F, Manchester JK, Semenkovich CF, Gordon Jl. Mechanisms underlying the resistance to diet-induced obesity in germ-free mice. Proceedings of the National Academy of Sciences of the United States of America 2007;104:979-84.

31. Barlow GM, Yu A, Mathur R. Role of the Gut Microbiome in Obesity and Diabetes Mellitus. Nutrition in clinical practice : official publication of the American Society for Parenteral and Enteral Nutrition 2015;30:787-97.

32. Cox LM, Blaser MJ. Pathways in microbe-induced obesity. Cell metabolism 2013;17:883-94.

33. Jones ML, Martoni CJ, Ganopolsky JG, Labbe A, Prakash S. The human microbiome and bile acid metabolism: dysbiosis, dysmetabolism, disease and intervention. Expert opinion on biological therapy 2014;14:467-82.

34. Arrieta $M C$, Bistritz $L$, Meddings JB. Alterations in intestinal permeability. Gut 2006;55:1512-20.

35. Human Microbiome Project C. A framework for human microbiome research. Nature 2012;486:215-21.

36. Human Microbiome Project C. Structure, function and diversity of the healthy human microbiome. Nature 2012;486:207-14.

37. Backhed F, Ley RE, Sonnenburg JL, Peterson DA, Gordon Jl. Host-bacterial mutualism in the human intestine. Science 2005;307:1915-20.

38. Gill SR, Pop M, Deboy RT, et al. Metagenomic analysis of the human distal gut microbiome. Science 2006;312:1355-9.

39. Aagaard K, Ma J, Antony KM, Ganu R, Petrosino J, Versalovic J. The placenta harbors a unique microbiome. Science translational medicine 2014;6:237ra65.

40. Claesson MJ, Jeffery IB, Conde S, et al. Gut microbiota composition correlates with diet and health in the elderly. Nature 2012;488:178-84.

41. Kerr CA, Grice DM, Tran CD, et al. Early life events influence whole-of-life metabolic health via gut microflora and gut permeability. Critical reviews in microbiology 2015;41:326-40.

42. de Meij TG, Budding AE, de Groot EF, et al. Composition and stability of intestinal microbiota of healthy children within a Dutch population. FASEB journal : official publication of the Federation of American Societies for Experimental Biology 2016;30:1512-22.

43. Yatsunenko T, Rey FE, Manary MJ, et al. Human gut microbiome viewed across age and geography. Nature 2012;486:222-7.

44. Smith EA, Macfarlane GT. Dissimilatory amino Acid metabolism in human colonic bacteria. Anaerobe 1997;3:327-37.

45. Swanson HI. Drug Metabolism by the Host and Gut Microbiota: A Partnership or Rivalry? Drug metabolism and disposition: the biological fate of chemicals 2015;43:1499-504. 
46. Conly JM, Stein K, Worobetz L, Rutledge-Harding S. The contribution of vitamin $\mathrm{K}_{2}$ (menaquinones) produced by the intestinal microflora to human nutritional requirements for vitamin $\mathrm{K}$. The American journal of gastroenterology 1994;89:915-23.

47. Goodrich JK, Waters JL, Poole AC, et al. Human genetics shape the gut microbiome. Cell 2014;159:789-99.

48. Koren O, Goodrich JK, Cullender TC, et al. Host remodeling of the gut microbiome and metabolic changes during pregnancy. Cell 2012;150:470-80.

49. Dominguez-Bello MG, Costello EK, Contreras M, et al. Delivery mode shapes the acquisition and structure of the initial microbiota across multiple body habitats in newborns. Proceedings of the National Academy of Sciences of the United States of America 2010;107:11971-5.

50. Dominguez-Bello MG, De Jesus-Laboy KM, Shen $\mathrm{N}$, et al. Partial restoration of the microbiota of cesarean-born infants via vaginal microbial transfer. Nature medicine 2016;22:250-3.

51. Avershina $E$, Lundgard $K$, Sekelja $M$, et al. Transition from infant- to adult-like gut microbiota. Environmental microbiology 2016.

52. Fallani M, Young D, Scott J, et al. Intestinal microbiota of 6-week-old infants across Europe: geographic influence beyond delivery mode, breast-feeding, and antibiotics. Journal of pediatric gastroenterology and nutrition 2010;51:77-84.

53. Rautava S, Luoto R, Salminen S, Isolauri E. Microbial contact during pregnancy, intestinal colonization and human disease. Nature reviews Gastroenterology \& hepatology 2012;9:565-76.

54. Grzeskowiak L, Collado MC, Mangani C, et al. Distinct gut microbiota in southeastern African and northern European infants. Journal of pediatric gastroenterology and nutrition 2012;54:812-6.

55. Jangi S, Lamont JT. Asymptomatic colonization by Clostridium difficile in infants: implications for disease in later life. Journal of pediatric gastroenterology and nutrition 2010;51:2-7.

56. Scott Fl, Horton DB, Mamtani R, et al. Administration of Antibiotics to Children Before Age 2 Years Increases Risk for Childhood Obesity. Gastroenterology 2016.

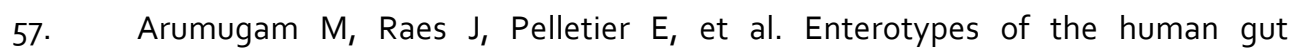
microbiome. Nature 2011;473:174-80.

58. Flint HJ, Bayer EA, Rincon MT, Lamed R, White BA. Polysaccharide utilization by gut bacteria: potential for new insights from genomic analysis. Nature reviews Microbiology 2008;6:121-31.

59. Bingham SA, Day NE, Luben $R$, et al. Dietary fibre in food and protection against colorectal cancer in the European Prospective Investigation into Cancer and Nutrition (EPIC): an observational study. Lancet 2003;361:1496-501. 
6o. Qin J, Li Y, Cai Z, et al. A metagenome-wide association study of gut microbiota in type 2 diabetes. Nature 2012;490:55-60.

61. Larsen N, Vogensen FK, van den Berg FW, et al. Gut microbiota in human adults with type 2 diabetes differs from non-diabetic adults. PloS one 2010;5:ego85.

62. Cani PD, Neyrinck AM, Fava F, et al. Selective increases of bifidobacteria in gut microflora improve high-fat-diet-induced diabetes in mice through a mechanism associated with endotoxaemia. Diabetologia 2007;50:2374-83.

63. Fukuda $\mathrm{S}$, Toh $\mathrm{H}$, Hase $\mathrm{K}$, et al. Bifidobacteria can protect from enteropathogenic infection through production of acetate. Nature 2011;469:543-7.

64. Shin NR, Whon TW, Bae JW. Proteobacteria: microbial signature of dysbiosis in gut microbiota. Trends in biotechnology 2015;33:496-503.

65. Samuel BS, Hansen EE, Manchester JK, et al. Genomic and metabolic adaptations of Methanobrevibacter smithii to the human gut. Proceedings of the National Academy of Sciences of the United States of America 2007;104:10643-8.

66. Backhed $F$, Ding $H$, Wang $T$, et al. The gut microbiota as an environmental factor that regulates fat storage. Proceedings of the National Academy of Sciences of the United States of America 2004;101:15718-23.

67. Turnbaugh PJ, Ley RE, Mahowald MA, Magrini V, Mardis ER, Gordon JI. An obesity-associated gut microbiome with increased capacity for energy harvest. Nature 2006;444:1027-31.

68. Ley RE, Backhed F, Turnbaugh P, Lozupone CA, Knight RD, Gordon Jl. Obesity alters gut microbial ecology. Proceedings of the National Academy of Sciences of the United States of America 2005;102:11070-5.

69. Ley RE, Turnbaugh PJ, Klein S, Gordon Jl. Microbial ecology: human gut microbes associated with obesity. Nature 2006;444:1022-3.

70. Zhang $\mathrm{H}$, DiBaise JK, Zuccolo A, et al. Human gut microbiota in obesity and after gastric bypass. Proceedings of the National Academy of Sciences of the United States of America 2009;106:2365-70.

71. Duncan SH, Lobley GE, Holtrop $\mathrm{G}$, et al. Human colonic microbiota associated with diet, obesity and weight loss. International journal of obesity 2008;32:17204.

72. Schwiertz A, Taras D, Schafer $K$, et al. Microbiota and SCFA in lean and overweight healthy subjects. Obesity 2010;18:190-5.

73. Turnbaugh PJ, Hamady M, Yatsunenko T, et al. A core gut microbiome in obese and lean twins. Nature 2009;457:480-4. 
74. Million $M$, Maraninchi $M$, Henry $M$, et al. Obesity-associated gut microbiota is enriched in Lactobacillus reuteri and depleted in Bifidobacterium animalis and Methanobrevibacter smithii. International journal of obesity 2012;36:817-25.

75. Yassour M, Lim MY, Yun HS, et al. Sub-clinical detection of gut microbial biomarkers of obesity and type 2 diabetes. Genome medicine 2016;8:17.

76. Cani PD, Bibiloni $R$, Knauf $C$, et al. Changes in gut microbiota control metabolic endotoxemia-induced inflammation in high-fat diet-induced obesity and diabetes in mice. Diabetes 2008;57:1470-81.

77. Karlsson FH, Tremaroli V, Nookaew I, et al. Gut metagenome in European women with normal, impaired and diabetic glucose control. Nature 2013;498:99103.

78. Remely $M$, Aumueller $E$, Merold $C$, et al. Effects of short chain fatty acid producing bacteria on epigenetic regulation of $\mathrm{FFAR}_{3}$ in type 2 diabetes and obesity. Gene 2014;537:85-92.

79. F.S.Teixeira T, Grześkowiak ŁM, Salminen S, Laitinen K, Bressan J, Gouveia Peluzio MdC. Faecal levels of Bifidobacterium and Clostridium coccoides but not plasma lipopolysaccharide are inversely related to insulin and HOMA index in women. Clinical nutrition 2013;32:1017-22.

8o. Zhang $X$, Shen $D$, Fang $Z$, et al. Human gut microbiota changes reveal the progression of glucose intolerance. PloS one 2013;8:e71108.

81. Tolhurst G, Heffron $H$, Lam YS, et al. Short-chain fatty acids stimulate glucagonlike peptide-1 secretion via the G-protein-coupled receptor FFAR2. Diabetes 2012;61:364-71.

82. Waget $A$, Cabou $C$, Masseboeuf $M$, et al. Physiological and pharmacological mechanisms through which the DPP-4 inhibitor sitagliptin regulates glycemia in mice. Endocrinology 2011;152:3018-29.

83. Hayes MR, Mietlicki-Baase EG, Kanoski SE, De Jonghe BC. Incretins and amylin: neuroendocrine communication between the gut, pancreas, and brain in control of food intake and blood glucose. Annual review of nutrition 2014;34:237-60.

84. Chambers ES, Viardot A, Psichas A, et al. Effects of targeted delivery of propionate to the human colon on appetite regulation, body weight maintenance and adiposity in overweight adults. Gut 2015;64:1744-54.

85. McNeil NI. The contribution of the large intestine to energy supplies in man. The American journal of clinical nutrition 1984;39:338-42.

86. Royall D, Wolever TM, Jeejeebhoy $\mathrm{KN}$. Clinical significance of colonic fermentation. The American journal of gastroenterology 1990;85:1307-12.

87. Cariou B, van Harmelen K, Duran-Sandoval D, et al. The farnesoid X receptor modulates adiposity and peripheral insulin sensitivity in mice. The Journal of biological chemistry 2006;281:11039-49. 
88. Abdelkarim $\mathrm{M}$, Caron $\mathrm{S}$, Duhem $\mathrm{C}$, et al. The farnesoid $\mathrm{X}$ receptor regulates adipocyte differentiation and function by promoting peroxisome proliferatoractivated receptor-gamma and interfering with the Wnt/beta-catenin pathways. The Journal of biological chemistry 2010;285:36759-67.

89. Hardie DG. AMPK--sensing energy while talking to other signaling pathways. Cell metabolism 2014;20:939-52.

90. Peterson LW, Artis D. Intestinal epithelial cells: regulators of barrier function and immune homeostasis. Nature reviews Immunology 2014;14:141-53.

91. Cani PD, Amar J, Iglesias MA, et al. Metabolic endotoxemia initiates obesity and insulin resistance. Diabetes 2007;56:1761-72.

92. Ruiz AG, Casafont F, Crespo J, et al. Lipopolysaccharide-binding protein plasma levels and liver TNF-alpha gene expression in obese patients: evidence for the potential role of endotoxin in the pathogenesis of non-alcoholic steatohepatitis. Obesity surgery 2007;17:1374-80.

93. Vavassori P, Mencarelli A, Renga B, Distrutti E, Fiorucci S. The bile acid receptor FXR is a modulator of intestinal innate immunity. Journal of immunology 2009;183:6251-61.

94. Rosen ED, Spiegelman BM. What we talk about when we talk about fat. Cell 2014;156:20-44.

95. Teixeira TF, Collado MC, Ferreira CL, Bressan J, Peluzio Mdo C. Potential mechanisms for the emerging link between obesity and increased intestinal permeability. Nutrition research 2012;32:637-47.

96. Creely SJ, McTernan PG, Kusminski CM, et al. Lipopolysaccharide activates an innate immune system response in human adipose tissue in obesity and type 2 diabetes. American journal of physiology Endocrinology and metabolism 2007;292:E740-7.

97. Amar J, Serino $M$, Lange $C$, et al. Involvement of tissue bacteria in the onset of diabetes in humans: evidence for a concept. Diabetologia 2011;54:3055-61.

98. Ma X, Fan PX, Li LS, Qiao SY, Zhang GL, Li DF. Butyrate promotes the recovering of intestinal wound healing through its positive effect on the tight junctions. Journal of animal science 2012;90 Suppl 4:266-8.

99. Arpaia N, Campbell C, Fan X, et al. Metabolites produced by commensal bacteria promote peripheral regulatory T-cell generation. Nature 2013;504:451-5.

100. Furusawa Y, Obata Y, Fukuda S, et al. Commensal microbe-derived butyrate induces the differentiation of colonic regulatory T cells. Nature 2013;504:446-50.

101. Smith PM, Howitt MR, Panikov N, et al. The microbial metabolites, short-chain fatty acids, regulate colonic Treg cell homeostasis. Science 2013;341:569-73. 
102. Cox MA, Jackson J, Stanton $M$, et al. Short-chain fatty acids act as antiinflammatory mediators by regulating prostaglandin $\mathrm{E}(2)$ and cytokines. World journal of gastroenterology 2009;15:5549-57.

103. Le Poul E, Loison C, Struyf S, et al. Functional characterization of human receptors for short chain fatty acids and their role in polymorphonuclear cell activation. The Journal of biological chemistry 2003;278:25481-9.

104. Tedelind S, Westberg F, Kjerrulf M, Vidal A. Anti-inflammatory properties of the short-chain fatty acids acetate and propionate: a study with relevance to inflammatory bowel disease. World journal of gastroenterology 2007;13:2826-32.

105. Liu T, Li J, Liu Y, et al. Short-chain fatty acids suppress lipopolysaccharideinduced production of nitric oxide and proinflammatory cytokines through inhibition of NF-kappaB pathway in RAW264.7 cells. Inflammation 2012;35:167684 .

106. Thomas C, Auwerx J, Schoonjans K. Bile acids and the membrane bile acid receptor TGR5--connecting nutrition and metabolism. Thyroid : official journal of the American Thyroid Association 2008;18:167-74.

107. Fiorucci S, Mencarelli A, Palladino G, Cipriani S. Bile-acid-activated receptors: targeting TGR5 and farnesoid-X-receptor in lipid and glucose disorders. Trends in pharmacological sciences 2009;30:570-80.

108. Brestoff JR, Artis D. Commensal bacteria at the interface of host metabolism and the immune system. Nature immunology 2013;14:676-84.

109. Wang YD, Chen WD, Yu D, Forman BM, Huang W. The G-protein-coupled bile acid receptor, Gpbar1 (TGR5), negatively regulates hepatic inflammatory response through antagonizing nuclear factor kappa light-chain enhancer of activated $B$ cells (NF-kappaB) in mice. Hepatology 2011;54:1421-32.

110. Duboc $H$, Rajca S, Rainteau D, et al. Connecting dysbiosis, bile-acid dysmetabolism and gut inflammation in inflammatory bowel diseases. Gut 2013;62:531-9.

111. Serino M, Fernandez-Real JM, Garcia-Fuentes E, et al. The gut microbiota profile is associated with insulin action in humans. Acta diabetologica 2013;50:753-61.

112. Vrieze A, de Groot PF, Kootte RS, Knaapen M, van Nood E, Nieuwdorp M. Fecal transplant: a safe and sustainable clinical therapy for restoring intestinal microbial balance in human disease? Best practice \& research Clinical gastroenterology 2013;27:127-37.

113. Vrieze A, Out C, Fuentes $S$, et al. Impact of oral vancomycin on gut microbiota, bile acid metabolism, and insulin sensitivity. Journal of hepatology 2014;60:82431.

114. Mikkelsen KH, Frost M, Bahl MI, et al. Effect of Antibiotics on Gut Microbiota, Gut Hormones and Glucose Metabolism. PloS one 2015;10:e0142352. 
115. Membrez $M$, Blancher $F$, Jaquet $M$, et al. Gut microbiota modulation with norfloxacin and ampicillin enhances glucose tolerance in mice. FASEB journal : official publication of the Federation of American Societies for Experimental Biology 2008;22:2416-26.

116. Firouzi S, Barakatun-Nisak MY, Ismail A, Majid HA, Nor Azmi K. Role of probiotics in modulating glucose homeostasis: evidence from animal and human studies. International journal of food sciences and nutrition 2013;64:780-6.

117. Shin NR, Lee JC, Lee HY, et al. An increase in the Akkermansia spp. population induced by metformin treatment improves glucose homeostasis in diet-induced obese mice. Gut 2014;63:727-35.

118. Sun J, Buys NJ. Glucose- and glycaemic factor-lowering effects of probiotics on diabetes: a meta-analysis of randomised placebo-controlled trials. The British journal of nutrition 2016;115:1167-77.

119. Canfora EE, Jocken JW, Blaak EE. Short-chain fatty acids in control of body weight and insulin sensitivity. Nature reviews Endocrinology 2015;11:577-91.

120. Evans K, Burdge GC, Wootton SA, Clark ML, Frayn KN. Regulation of dietary fatty acid entrapment in subcutaneous adipose tissue and skeletal muscle. Diabetes 2002;51:2684-90.

121. Miles JM, Park YS, Walewicz D, et al. Systemic and forearm triglyceride metabolism: fate of lipoprotein lipase-generated glycerol and free fatty acids. Diabetes 2004;53:521-7.

122. Sukonina V, Lookene A, Olivecrona T, Olivecrona G. Angiopoietin-like protein 4 converts lipoprotein lipase to inactive monomers and modulates lipase activity in adipose tissue. Proceedings of the National Academy of Sciences of the United States of America 2006;103:17450-5.

123. Kersten S. Physiological regulation of lipoprotein lipase. Biochimica et biophysica acta 2014;1841:919-33.

124. Dijk W, Kersten S. Regulation of lipoprotein lipase by Angptl4. Trends in endocrinology and metabolism: TEM 2014;25:146-55.

125. Cristancho AG, Lazar MA. Forming functional fat: a growing understanding of adipocyte differentiation. Nature reviews Molecular cell biology 2011;12:722-34.

126. Dijk W, Beigneux AP, Larsson M, Bensadoun A, Young SG, Kersten S. Angiopoietin-like 4 (ANGPTL4) promotes intracellular degradation of lipoprotein lipase in adipocytes. Journal of lipid research 2016.

127. Gray NE, Lam LN, Yang K, Zhou AY, Koliwad S, Wang JC. Angiopoietin-like 4 (Angpt/4) protein is a physiological mediator of intracellular lipolysis in murine adipocytes. The Journal of biological chemistry 2012;287:8444-56.

128. Jocken JW, Blaak EE. Catecholamine-induced lipolysis in adipose tissue and skeletal muscle in obesity. Physiology \& behavior 2008;94:219-30. 
129. Laurent C, Simoneau C, Marks L, et al. Effect of acetate and propionate on fasting hepatic glucose production in humans. European journal of clinical nutrition 1995;49:484-91.

130. Fernandes J, Vogt J, Wolever TM. Intravenous acetate elicits a greater free fatty acid rebound in normal than hyperinsulinaemic humans. European journal of clinical nutrition 2012;66:1029-34.

131. Crouse JR, Gerson CD, DeCarli LM, Lieber CS. Role of acetate in the reduction of plasma free fatty acids produced by ethanol in man. Journal of lipid research 1968;9:509-12.

132. Zaibi MS, Stocker CJ, O'Dowd J, et al. Roles of GPR41 and GPR43 in leptin secretory responses of murine adipocytes to short chain fatty acids. FEBS letters 2010;584:2381-6.

133. Rizzo G, Disante M, Mencarelli A, et al. The farnesoid X receptor promotes adipocyte differentiation and regulates adipose cell function in vivo. Molecular pharmacology 2006;70:1164-73.

134. Hong $Y H$, Nishimura $Y$, Hishikawa $D$, et al. Acetate and propionate short chain fatty acids stimulate adipogenesis via GPCR43. Endocrinology 2005;146:5092-9.

135. Szabo G, Bala S, Petrasek J, Gattu A. Gut-liver axis and sensing microbes. Digestive diseases 2010;28:737-44.

136. Burcelin R, Serino $M$, Chabo $C$, et al. Metagenome and metabolism: the tissue microbiota hypothesis. Diabetes, obesity \& metabolism 2013;15 Suppl 3:61-70.

137. Zhu L, Baker SS, Gill C, et al. Characterization of gut microbiomes in nonalcoholic steatohepatitis (NASH) patients: a connection between endogenous alcohol and NASH. Hepatology 2013;57:601-9.

138. Velagapudi VR, Hezaveh R, Reigstad CS, et al. The gut microbiota modulates host energy and lipid metabolism in mice. Journal of lipid research 2010;51:110112.

139. den Besten G, van Eunen K, Groen AK, Venema K, Reijngoud DJ, Bakker BM. The role of short-chain fatty acids in the interplay between diet, gut microbiota, and host energy metabolism. Journal of lipid research 2013;54:2325-40.

140. Li T, Owsley E, Matozel M, Hsu P, Novak CM, Chiang JY. Transgenic expression of cholesterol 7alpha-hydroxylase in the liver prevents high-fat diet-induced obesity and insulin resistance in mice. Hepatology 2010;52:678-90.

141. Thiebaud D, Jacot E, DeFronzo RA, Maeder E, Jequier E, Felber JP. The effect of graded doses of insulin on total glucose uptake, glucose oxidation, and glucose storage in man. Diabetes 1982;31:957-63.

142. Leto $D$, Saltiel AR. Regulation of glucose transport by insulin: traffic control of GLUT4. Nature reviews Molecular cell biology 2012;13:383-96. 
143. Liang $\mathrm{H}$, Hussey $\mathrm{SE}$, Sanchez-Avila A, Tantiwong $\mathrm{P}$, Musi N. Effect of lipopolysaccharide on inflammation and insulin action in human muscle. PloS one 2013;8:e63983.

144. Pillon NJ, Bilan PJ, Fink LN, Klip A. Cross-talk between skeletal muscle and immune cells: muscle-derived mediators and metabolic implications. American journal of physiology Endocrinology and metabolism 2013;304:E453-65.

145. Parseus A, Sommer N, Sommer F, et al. Microbiota-induced obesity requires farnesoid $X$ receptor. Gut 2016.

146. Watanabe $M$, Houten SM, Mataki $C$, et al. Bile acids induce energy expenditure by promoting intracellular thyroid hormone activation. Nature 2006;439:484-9.

147. Farese RV, Jr., Yost TJ, Eckel RH. Tissue-specific regulation of lipoprotein lipase activity by insulin/glucose in normal-weight humans. Metabolism: clinical and experimental 1991;40:214-6.

148. Moors CC, van der Zijl NJ, Diamant M, Blaak EE, Goossens GH. Impaired insulin sensitivity is accompanied by disturbances in skeletal muscle fatty acid handling in subjects with impaired glucose metabolism. International journal of obesity 2012;36:709-17.

149. Blaak EE, Wagenmakers AJ, Glatz JF, et al. Plasma FFA utilization and fatty acidbinding protein content are diminished in type 2 diabetic muscle. American journal of physiology Endocrinology and metabolism 2000;279:E146-54.

150. Chai W, Dong $Z$, Wang $N$, et al. Glucagon-like peptide 1 recruits microvasculature and increases glucose use in muscle via a nitric oxidedependent mechanism. Diabetes 2012;61:888-96.

151. Cherbut $C$, Ferrier $L$, Roze $C$, et al. Short-chain fatty acids modify colonic motility through nerves and polypeptide $Y Y$ release in the rat. The American journal of physiology 1998;275:G1415-22.

152. Samuel BS, Shaito A, Motoike T, et al. Effects of the gut microbiota on host adiposity are modulated by the short-chain fatty-acid binding $G$ protein-coupled receptor, Gpr41. Proceedings of the National Academy of Sciences of the United States of America 2008;105:16767-72.

153. Bonini JA, Anderson SM, Steiner DF. Molecular cloning and tissue expression of a novel orphan $G$ protein-coupled receptor from rat lung. Biochemical and biophysical research communications 1997;234:190-3.

154. Cornall LM, Mathai ML, Hryciw DH, McAinch AJ. Diet-induced obesity upregulates the abundance of GPR43 and GPR120 in a tissue specific manner. Cellular physiology and biochemistry : international journal of experimental cellular physiology, biochemistry, and pharmacology 2011;28:949-58.

155. Khan MT, Nieuwdorp M, Backhed F. Microbial modulation of insulin sensitivity. Cell metabolism 2014;20:753-60. 
156. Li Y, Cao X, Li LX, Brubaker PL, Edlund H, Drucker DJ. beta-Cell Pdxı expression is essential for the glucoregulatory, proliferative, and cytoprotective actions of glucagon-like peptide-1. Diabetes 2005;54:482-91.

157. Balkan B, Li X. Portal GLP-1 administration in rats augments the insulin response to glucose via neuronal mechanisms. American journal of physiology Regulatory, integrative and comparative physiology 2000;279:R1449-54.

158. Steinert RE, Peterli R, Keller $S$, et al. Bile acids and gut peptide secretion after bariatric surgery: a 1-year prospective randomized pilot trial. Obesity 2013;21:E660-8.

159. McNelis JC, Lee YS, Mayoral R, et al. GPR43 Potentiates beta-Cell Function in Obesity. Diabetes 2015;64:3203-17.

16o. Popescu IR, Helleboid-Chapman A, Lucas A, et al. The nuclear receptor FXR is expressed in pancreatic beta-cells and protects human islets from lipotoxicity. FEBS letters 2010;584:2845-51.

161. Renga B, Mencarelli A, Vavassori P, Brancaleone V, Fiorucci S. The bile acid sensor FXR regulates insulin transcription and secretion. Biochimica et biophysica acta 2010;1802:363-72.

162. Lozupone CA, Stombaugh JI, Gordon JI, Jansson JK, Knight R. Diversity, stability and resilience of the human gut microbiota. Nature 2012;489:220-30.

163. Kashyap PC, Marcobal A, Ursell LK, et al. Complex interactions among diet, gastrointestinal transit, and gut microbiota in humanized mice. Gastroenterology 2013;144:967-77.

164. Zhang L, Li HT, Shen L, Fang QC, Qian LL, Jia WP. Effect of Dietary Resistant Starch on Prevention and Treatment of Obesity-related Diseases and Its Possible Mechanisms. Biomedical and environmental sciences : BES 2015;28:291-7.

165. de Vrese M, Schrezenmeir J. Probiotics, prebiotics, and synbiotics. Advances in biochemical engineering/biotechnology 2008;111:1-66.

166. Delzenne NM, Neyrinck AM, Backhed F, Cani PD. Targeting gut microbiota in obesity: effects of prebiotics and probiotics. Nature reviews Endocrinology 2011;7:639-46.

167. Gerritsen J, Smidt H, Rijkers GT, de Vos WM. Intestinal microbiota in human health and disease: the impact of probiotics. Genes \& nutrition 2011;6:209-40.

168. David LA, Materna AC, Friedman J, et al. Host lifestyle affects human microbiota on daily timescales. Genome biology 2014;15:R89.

169. David LA, Maurice CF, Carmody RN, et al. Diet rapidly and reproducibly alters the human gut microbiome. Nature 2014;505:559-63. 
170. Perez-Cobas $A E$, Artacho $A$, Knecht $H$, et al. Differential effects of antibiotic therapy on the structure and function of human gut microbiota. PloS one 2013;8:e80201.

171. Willing BP, Russell SL, Finlay BB. Shifting the balance: antibiotic effects on hostmicrobiota mutualism. Nature reviews Microbiology 2011;9:233-43.

172. Sayin SI, Wahlstrom A, Felin J, et al. Gut microbiota regulates bile acid metabolism by reducing the levels of tauro-beta-muricholic acid, a naturally occurring FXR antagonist. Cell metabolism 2013;17:225-35.

173. Romick-Rosendale LE, Goodpaster AM, Hanwright PJ, et al. NMR-based metabonomics analysis of mouse urine and fecal extracts following oral treatment with the broad-spectrum antibiotic enrofloxacin (Baytril). Magnetic resonance in chemistry: MRC 2009;47 Suppl 1:S36-46.

174. Woodmansey EJ, McMurdo ME, Macfarlane GT, Macfarlane S. Comparison of compositions and metabolic activities of fecal microbiotas in young adults and in antibiotic-treated and non-antibiotic-treated elderly subjects. Applied and environmental microbiology 2004;70:6113-22.

175. Yap IK, Li JV, Saric J, et al. Metabonomic and microbiological analysis of the dynamic effect of vancomycin-induced gut microbiota modification in the mouse. Journal of proteome research 2008;7:3718-28.

176. Gustafson $\mathrm{RH}$, Bowen RE. Antibiotic use in animal agriculture. Journal of applied microbiology 1997;83:531-41.

177. Cox LM, Blaser MJ. Antibiotics in early life and obesity. Nature reviews Endocrinology 2015;11:182-90.

178. Mikkelsen KH, Knop FK, Frost M, Hallas J, Pottegard A. Use of Antibiotics and Risk of Type 2 Diabetes: A Population-Based Case-Control Study. The Journal of clinical endocrinology and metabolism 2015;100:3633-40.

179. Chou CJ, Membrez M, Blancher F. Gut decontamination with norfloxacin and ampicillin enhances insulin sensitivity in mice. Nestle Nutrition workshop series Paediatric programme 2008;62:127-37; discussion 37-40.

180. Hwang I, Park YJ, Kim YR, et al. Alteration of gut microbiota by vancomycin and bacitracin improves insulin resistance via glucagon-like peptide 1 in diet-induced obesity. FASEB journal : official publication of the Federation of American Societies for Experimental Biology 2015;29:2397-411. 


\section{Effects of gut microbiota manipulation by antibiotics on host metabolism in obese humans: a randomized double- blind placebo-controlled trial}

Dorien Reijnders

Gijs H Goossens Gerben D A Hermes

Evelien P J G Neis

Christina M van der Beek Jasper Most Jens J Holst

Kaatje Lenaerts Ruud S Kootte Max Nieuwdorp Albert K Groen Steven W M Olde Damink Mark V Boekschoten

Hauke Smidt Erwin G Zoetendal Cornelis H C Dejong Ellen E Blaak 


\section{Abstract}

The gut microbiota has been implicated in obesity and cardiometabolic diseases, although evidence in humans is scarce. We investigated how gut microbiota manipulation by antibiotics (7-day administration of amoxicillin, vancomycin or placebo) affects host metabolism in 57 obese, prediabetic men. Vancomycin, but not amoxicillin, decreased bacterial diversity and reduced Firmicutes involved in short-chain fatty acid and bile acid metabolism, concomitant with altered plasma and/or fecal metabolite concentrations. Adipose tissue gene expression of oxidative pathways was upregulated by antibiotics, whereas immune-related pathways were downregulated by vancomycin. Antibiotics did not affect tissue-specific insulin sensitivity, energy/substrate metabolism, postprandial hormones and metabolites, systemic inflammation, gut permeability and adipocyte size. Importantly, energy harvest, adipocyte size and whole-body insulin sensitivity were not altered at 8-weeks follow-up, despite a still considerably altered microbial composition, indicating that interference with adult microbiota by 7 -days antibiotic treatment has no clinically relevant impact on metabolic health in obese humans. 


\section{Introduction}

Accumulating evidence indicates that the composition of the gut microbiota plays a prominent role in body weight regulation and the development of type 2 diabetes mellitus ${ }^{1,2}$. The gut microbiota regulates energy extraction from otherwise indigestible carbohydrates, determines the integrity of the intestinal epithelial layer, and influences the production and absorption of multiple signaling molecules involved in host metabolism. Several studies have demonstrated that germ-free mice are protected from diet-induced obesity, lowgrade inflammation and glucose intolerance as compared to conventionally raised animals ${ }^{3 / 4}$. Furthermore, it has been shown that transferring microbiota via fecal transplantation evoked alterations in body weight and insulin sensitivity in both rodents ${ }^{4}$ and humans ${ }^{5,6}$ Taken together, these data indicate that modulation of the gut microbiota may provide a promising avenue to target obesity-related metabolic disorders ${ }^{7}$.

The gut microbiota composition can be modulated by, amongst others, prebiotics, probiotics and antibiotics ${ }^{8}$, thereby altering the presence and expression of microbial genes and derived metabolites such as bile acids (BA) and short-chain fatty acids (SCFA) $)^{9,10}$. Particularly, the use of antibiotics has been associated with increased metabolic impairments, mainly when exposure occurs in early life ${ }^{11,12}$. Of note, these findings are primarily based on animal studies, in which the animals have mostly been exposed to a combination of antibiotics for periods varying from two to twenty weeks ${ }^{13-17}$. It has recently been shown that antibiotics may improve peripheral insulin sensitivity in a small number of obese subjects ${ }^{18}$. Nevertheless, the effects observed in the latter study were relatively minor and, importantly, the study was not placebo-controlled. Thus, wellcontrolled, large human studies examining the effects and underlying mechanisms of microbiota modulation on host metabolism are currently lacking.

Here, we report on a randomized, double-blind, placebo-controlled trial that was designed to investigate the effects of broad and narrow-spectrum antibiotic treatment for seven days on gut microbiota composition, tissue-specific insulin sensitivity, energy expenditure, substrate oxidation, fecal and plasma BA and SCFA concentrations, gut permeability, abdominal subcutaneous adipocyte size and systemic low-grade inflammation in obese men with impaired glucose homeostasis. Moreover, eight weeks after cessation of antibiotic treatment, we 
again determined microbiota composition, whole-body insulin sensitivity (HOMA-IR), fecal energy harvest and adipocyte size.

\section{Methods}

\section{Study participants}

57 low active ( $<3 \mathrm{~h}$ organized sports activities per week), weight-stable ( $<2 \mathrm{~kg}$ body weight change 3 months prior to inclusion) overweight/obese (BMI 25-35 $\left.\mathrm{kg} / \mathrm{m}^{2}\right)$, Caucasian men, between 35 and 70 years with impaired glucose metabolism (either fasting glucose $>6.1 \mathrm{mmol} / \mathrm{l}$, and/or $2 \mathrm{~h}$ glucose between 7.8 $11 \mathrm{mmol} / \mathrm{l}$ ) and HOMA-IR>2.2 were included in this study (ClinicalTrials.gov, NCTo2241421). Subjects were recruited via advertisements in local newspapers, and were all living in the area around Maastricht, The Netherlands. All subjects gave written informed consent for participation in this study, which was reviewed and approved by the local Medical Ethical Committee of Maastricht University Medical Center+. All procedures were according to the declaration of Helsinki (revised version, October 2008). Exclusion criteria were: the use of antibiotics for a period of three months before entering the study, known allergic reactions to any type of antibiotics, hearing disorders, cancer, liver malfunction, major illnesses with a life expectancy less than five years and pulmonary, hepatic, cardiovascular, kidney, and gastrointestinal disease. Subjects did not use $\beta$ blockers, lipid and glucose lowering-drugs, anti-oxidants or chronic corticosteroids.

\section{Study design \& randomization}

This randomized, placebo-controlled, double-blind study had a three-armed parallel design. Participants were randomized to oral intake of amoxicillin (broadspectrum antibiotic), vancomycin (directed against Gram-positive bacteria) or placebo (microcrystalline cellulose) for seven consecutive days $(1500 \mathrm{mg} / \mathrm{d}$ ). Antibiotics and placebo were equally capsulated to blind the content to subjects and investigators (BasicPharma, The Netherlands). The allocation sequence was established by computer-generated randomization (https://nl.tenalea.net). Block-randomization with stratification for BMl, age and $2 \mathrm{~h}$-glucose values was used to increase the homogeneity of the treatment arms (block size, $n=6$ ). After completion of the study, returned capsules were counted to assess compliance. Participants were asked to maintain their habitual physical activity pattern and 
dietary habits (monitored by 3-day food diaries) throughout the study. The evening before an investigation day, a low fiber, low fat meal was consumed. Before and after intervention, study measurements were conducted following a 10-h overnight fast. To ensure complete systemic and gastrointestinal clearance of antibiotics, a 2-day wash-out period was taken into account before posttreatment measurements. Participants returned for a follow-up visit eight weeks after treatment cessation.

\section{Hyperinsulinemic-euglycemic clamp}

A two-step hyperinsulinemic-euglycemic clamp combined with a $\left[6,6-{ }^{2} \mathrm{H}_{2}\right]-$ glucose tracer (Cambridge Isotope Laboratories, Andover, MA, USA) was performed to measure Rd, EGP ${ }^{41}$ and the insulin-mediated suppression of FFA ${ }^{42,43}$. Blood samples were taken from a superficial dorsal hand vein, which was arterialized by using a hot-box $\left(\sim 50^{\circ} \mathrm{C}\right)$. After a bolus-injection $(2.4 \mathrm{mg} / \mathrm{kg})$, tracer-infusion was started at $0.04 \mathrm{mg} / \mathrm{kg} / \mathrm{min}$, which was continued throughout the measurement. After $2 \mathrm{~h}$, low-dose insulin was infused at $10 \mathrm{mU} / \mathrm{m}^{2} / \mathrm{min}$ for 2 $\mathrm{h}^{44}$, followed by high-dose insulin at $40 \mathrm{mU} / \mathrm{m}^{2} / \mathrm{min}$ for $2 \mathrm{~h}^{45}$. By variable coinfusion of a $17.5 \%$-glucose solution, enriched by $1.1 \%$ tracer, plasma glucose concentrations were maintained at $5.0 \mathrm{mmol} / \mathrm{l}$. For calculation of steady-statekinetics, additional blood samples were taken in the last 30 min of each step (o, 10 and $40 \mathrm{mU} / \mathrm{m}^{2} / \mathrm{min}$ insulin).

\section{Postprandial test}

Blood was sampled from a superficial dorsal hand vein, which was arterialized by placing the hand into a hot-box $\left(\sim 5^{\circ}{ }^{\circ} \mathrm{C}\right)$. Blood samples were taken during the fasting state ( $t-30, t-15$, to $\mathrm{min}$ ) and postprandial $(t=30,60,90,120,180$ and 240 min) after ingestion of the test meal. The liquid test meal, that was consumed within 5 min, provided $2.6 \mathrm{MJ}$ (61 E\% fat, $33 \mathrm{E} \%$ carbohydrate, $6 \mathrm{E} \%$ protein), which was consumed within 5 min at $\mathrm{t}=\mathrm{O}^{46}$.

\section{Indirect calorimetry}

For indirect calorimetry during fasting (30 $\mathrm{min}$ ) and the 4 -h postprandial state, the open-circuit ventilated hood system was used (Omnical, Maastricht University, Maastricht, Netherlands) ${ }^{47}$. Calculations of energy expenditure and substrate oxidation were performed according to the formulas of Weir ${ }^{48}$ and 
$\mathrm{Frayn}^{49}$. Nitrogen excretion was based on the assumption that protein oxidation represents $\sim 15 \%$ of total energy expenditure ${ }^{50}$.

\section{Gut Permeability test}

After baseline urine collection, subjects drank a $150 \mathrm{ml}$ multisaccharide test mix [1 g sucrose (Van Gilse, Dinteloord, the Netherlands), $1 \mathrm{~g}$ lactulose (Centrafarm, Etten-Leur, the Netherlands), $1 \mathrm{~g}$ sucralose (Brenntag, Sittard, the Netherlands), $1 \mathrm{~g}$ erythritol (Danisco Sweeteners, Copenhagen, Denmark), $0.5 \mathrm{~g}$ of I-rhamnose $(\text { Danisco) }]^{29}$. Urine was collected for determination of the urinary sucrose concentration in the $0-120$ min urine collection, representing gastro-duodenal permeability, whereas in this collection small intestinal permeability is represented by the lactulose/rhamnose ratio. Proximal colon permeability is represented by the suclarose/erythritol ratio of the 120-300 min urine collection.

\section{Biochemical analyses for plasma variables}

Blood was collected into pre-chilled tubes, centrifuged at $1000 \mathrm{~g}$, and plasma was snap-frozen and stored at $-80^{\circ} \mathrm{C}$ until analyses. Isotopic enrichment of plasma glucose was determined by electron ionization gas chromatography-mass spectrometry and expressed as tracer-to-tracee ratio for steady-state calculations of $\mathrm{Rd}$ and $\mathrm{EGP}^{41}$. Plasma glucose, lactate, FFA and glycerol were determined with the Cobas Fara auto-analyzer (Roche, Switzerland). Plasma insulin was measured with a double antibody radioimmunoassay (Millipore, MA, USA). Plasma leptin concentrations were analyzed using commercially available radioimmunoassay kits (Human Leptin RIA, Millipore Corporation, Billerica, MA, USA). Plasma ANGPTL 4 concentrations were measured by ELISA as described ${ }^{51}$. Plasma concentrations of IL-6, IL-8 and TNF- $\alpha$ were determined using a multiplex enzyme-linked immuno-sorbent assay (Human Prolnflammatory II 4-Plex UltraSensitive Kit, Meso Scale Diagnositics, Rockville, MD, USA). Isocratic ionexchange HPLC (Model PU-1980 pump, Jasco Easton, MD) with mass spectrometry (Model LTO XL, Thermo Fisher Scientific, Waltham, MA) was used to determine sugar concentrations in plasma and urine for gastrointestinal permeability assessment ${ }^{29}$. LPS-binding protein was measured using noncommercial ELISA as described before ${ }^{52}$. Plasma concentrations of GLP-1 were measured by radioimmunoassays as previously described ${ }^{53}$. Plasma BA profile was measured using liquid chromatography tandem mass spectrometry (LC- 
MS/MS) ${ }^{18}$. The total amount of primary (cholic acid and chenodeoxycholic acid and their taurine and glycine conjugated forms) and secondary BA (deoxycholic acid, lithocholic acid and their conjugated forms) was calculated as the sum of the individually quantified BA. Plasma SCFA were determined by LC-MS/MS as reported before ${ }^{54}$. The detection limits for acetate, propionate and butyrate were $0.1,0.05$ and $0.05 \mu \mathrm{mol} / \mathrm{L}$, respectively.

\section{Laboratory analysis of adipose tissue}

Abdominal subcutaneous AT biopsies were taken under local anesthesia under fasted conditions. One portion was embedded in paraffin. Sections were cut for staining, digital imaging and computerized morphometric measurement of individual adipocytes55. One portion $(\sim 500 \mathrm{mg})$ was snapfrozen in liquid nitrogen, from which RNA was extracted (Trizol chloroform extraction, Invitrogen, Cergy Pontoise, France) and used for microarray analysis. $100 \mathrm{ng}$ total RNA was labeled by Whole-Transcript Sense Target Assay and hybridized to human wholegenome Affymetrix Gene 1.1 ST arrays, targeting 19793 unique genes (Affymetrix, Santa Clara, CA, USA). Quality control and data analysis pipeline have been described in detail previously56. Individual genes on the array were defined as changed when comparison of the normalized signal intensities showed a FDRq<0.05 in a two-tailed paired t-test with Bayesan correction (Limma)57. Further functional data analysis was performed on the filtered data set with Gene Set Enrichment Analysis (GSEA, http://www.broad.mit.edu/gsea). Gene sets were selected based upon FDRq $<0.2$. Array data have been submitted to the Gene Expression Omnibus: number GSE76003.

\section{Laboratory analysis of feces}

Feces was collected at home for two consecutive days at baseline, seven days and eight weeks after intervention using the BMP commode specimen collection system, and divided over sterile tubes at home. Subjects were provided with a box of dry ice to freeze their stool samples immediately after defecation at approximately $-80^{\circ} \mathrm{C}$ and for transport to the university. Total fecal amount was weighed, and $24^{-h}$ fecal samples were used to determine energy content using adiabatic bomb calorimetry (CBB 330, standard benzoic acid $6320 \mathrm{cal} / \mathrm{g}$, BCSCRMnogoN). 24-h fecal BA composition was determined by using gas chromatography $(\mathrm{GC})$ as described before ${ }^{41}$. Fecal SCFA were measured by gas 
chromatography-mass spectrometry (GC-MS, Medical laboratory 'Dr. Stein \& Collegae' Germany), according to the method described before ${ }^{58}$.

For microbiota profiling, DNA was isolated from $24^{-h}$ fecal samples as described before $^{18}$ and subsequently used for phylogenetic profiling using the HITchip phylogenetic microarray ${ }^{19}$. Standardized quality control was maintained through our library of a duplicated set of 3,631 probes targeting the 16S rRNA gene sequences of over 1,000 intestinal bacterial phylotypes. A more detailed description of microbiota profiling procedures can be found in the Supplemental Experimental Procedures.

\section{Statistics}

The calculated sample size ( $n=19$ per treatment arm) was based on a $20 \%$ physiologically relevant change of insulin sensitivity $(\alpha=0.05, \beta=0.8)$. All data were evaluated for normality. Univariate analysis (ANOVA) was applied to compare group characteristics at baseline. Differences between treatments were analyzed using repeated-measures ANOVA with time and treatment as factors. ANCOVA analysis of the delta (post-pre value) was used for parameters when significantly different at baseline, taking the baseline value into account as covariate. The postprandial response (energy expenditure, substrate oxidation and GLP-1) is given as incremental area under the curve (iAUC/min), which was calculated by the trapezoid method. For HITchip analysis, logio-transformed signals were used as a proxy for bacterial logarithmic abundance. To determine which bacterial groups were significantly different in relative abundance before and after treatment within each group, a paired Wilcoxon test was used. Between-treatment group effects were assessed with linear mixed models using the Ime 4 package ${ }^{59}$. Benjamini-Hochberg correction was applied for multiple testing. We used Random Forests, a supervised machine-learning technique, and the pre and post treatment classes to confirm these results ${ }^{60}$. To determine whether individuals could be grouped into classes of specific metabolic responses to the interventions, we used the $\mathrm{Icmm} R$ package ${ }^{61}$ to perform Latent Class Analysis. Diversity of the microbiota was quantified based on non-logarithmized HITchip oligo-level signals by inverse Simpson's index using the Vegan package ${ }^{62}$. ANOVA with Tukey's Honest Significant post hoc analysis was applied to compare diversity between and within groups. Data are expressed as means \pm 
standard error of the mean (SEM), with a two-sided significance level of $P<0.05$. Statistical analysis was performed using SPSS 20.0 for Macintosh and R 3.03 .

\section{Results and Discussion}

\section{Subject characteristics}

To study the role of the gut microbiota, we randomized 57 overweight and obese 35-70 year old Caucasian men to oral administration of the broad-spectrum antibiotic amoxicillin (AMOX), narrow-spectrum antibiotic vancomycin (VANCO, directed against Gram-positive bacteria), or placebo (PLA) for seven days. No significant differences in baseline characteristics were present between groups (Table 1). All subjects had impaired fasting glucose levels (plasma glucose $\geq 5.6$ $\mathrm{mmol} / \mathrm{l}$ ) and/or impaired glucose tolerance ( $2 \mathrm{~h}$ plasma glucose during a $75 \mathrm{~g}$ oral glucose tolerance test 7.8-11.1 mmol/l), and were insulin resistant (homeostasis model assessment for insulin resistance; HOMA-IR>2.2). One subject randomized to the AMOX intervention was considered a dropout due to use of other antibiotics during the study period. No serious adverse events and only a few cases of mild gastrointestinal discomfort were reported. There were no differences in daily energy and macronutrient intake, as monitored by a threeday food diary, between and within groups before and after intervention. Furthermore, body weight remained unchanged for all treatment groups throughout the study period and at follow-up (data not shown).

Table 2.1. Baseline characteristics of the study population

\begin{tabular}{llll} 
Variable & PLA $(n=19)$ & AMOX $(n=18)$ & VANCO $(n=19)$ \\
\hline Age $($ years $)$ & $60.9 \pm 1.7$ & $55.7 \pm 1.5$ & $60.6 \pm 1.5$ \\
Body weight $(\mathrm{kg})$ & $96.7 \pm 2.3$ & $96.3 \pm 2.5$ & $97.6 \pm 1.9$ \\
Body mass index $\left(\mathrm{kg} / \mathrm{m}^{2}\right)$ & $31.0 \pm 0.5$ & $31.1 \pm 0.8$ & $31.5 \pm 0.6$ \\
Waist/Hip ratio & $1.04 \pm 0.01$ & $1.04 \pm 0.01$ & $1.07 \pm 0.01$ \\
Waist circumference $(\mathrm{cm})$ & $98.0 \pm 8.1$ & $101.1 \pm 6.4$ & $106.7 \pm 6.3$ \\
Fasting glucose $(\mathrm{mM})$ & $6.0 \pm 0.1$ & $6.1 \pm 0.1$ & $6.1 \pm 0.1$ \\
2h OGTT glucose $(\mathrm{mmol} / \mathrm{l})$ & $7.7 \pm 0.4$ & $7.0 \pm 0.5$ & $7.2 \pm 0.4$ \\
Fasting insulin $(\mathrm{mU} / \mathrm{l})$ & $15.7 \pm 1.5$ & $17.9 \pm 1.6$ & $16.8 \pm 1.1$ \\
HOMA-IR $_{\text {HbA }}(\%)$ & $4.2 \pm 0.4$ & $4.9 \pm 0.5$ & $4.6 \pm 0.3$ \\
\hline PluS-minus Valus & $5.5 \pm 0.1$ & $5.6 \pm 0.1$ & $5.6 \pm 0.1$
\end{tabular}

Plus-minus values are means \pm SEM. HOMA-IR denotes homeostasis model assessment of insulin resistance, $\mathrm{HbA}_{1 \mathrm{c}}$ glycated haemoglobin 


\section{Efficacy of microbiota manipulation by antibiotic treatment}

The fecal microbiota composition was determined by analyzing $16 \mathrm{~S}$ ribosomal RNA (rRNA) gene amplicons, using the Human Intestinal Tract Chip Microarray (HITchip) ${ }^{19}$, which showed that seven-day VANCO markedly decreased microbial diversity $(P<0.001)$, whereas this was not affected by AMOX $(P=0.42)$ as compared to PLA (Figure 2.1). VANCO decreased the relative abundance of mainly Gram-positive bacteria of the Firmicutes phylum. Among the most strongly affected groups were genus-like groups that contain known butyrateproducing species from Clostridium clusters IV and XIVa, such as Coprococcus eutactus, Faecalibacterium prausnitzii and Anaerostipes caccae, as well as species involved in BA dehydroxylation such as Clostridium leptum. Conversely, Gramnegative Proteobacteria, members of Clostridium cluster IX and VANCO-resistant Gram-positive bacilli such as Lactobacillus plantarum and Enterococcus, showed increased relative abundance after VANCO treatment (Figure 2.1 and Table S2.1), which is in line with previous studies ${ }^{18,20}$. This pattern was confirmed with a supervised machine-learning technique (Random Forests analysis, Table S2). Importantly, microbiota composition was still affected eight weeks after cessation of VANCO treatment. Microbial diversity was still lower $(q=0.053)$, and overall similarity and composition were deviant from baseline (pre-treatment) as compared to PLA. Although the bacterial groups that increased in abundance due to VANCO treatment had in general returned to baseline levels, several members of Clostridium clusters IV and XIVa were still decreased as compared to PLA. Furthermore, observed dynamics with respect to gut microbiota composition and diversity were individual-specific (Figure S2.1). In contrast, AMOX treatment did not affect microbiota composition after seven days treatment or at eight weeks follow-up compared to PLA, which is in accordance with a previous study in obese humans ${ }^{18}$. 


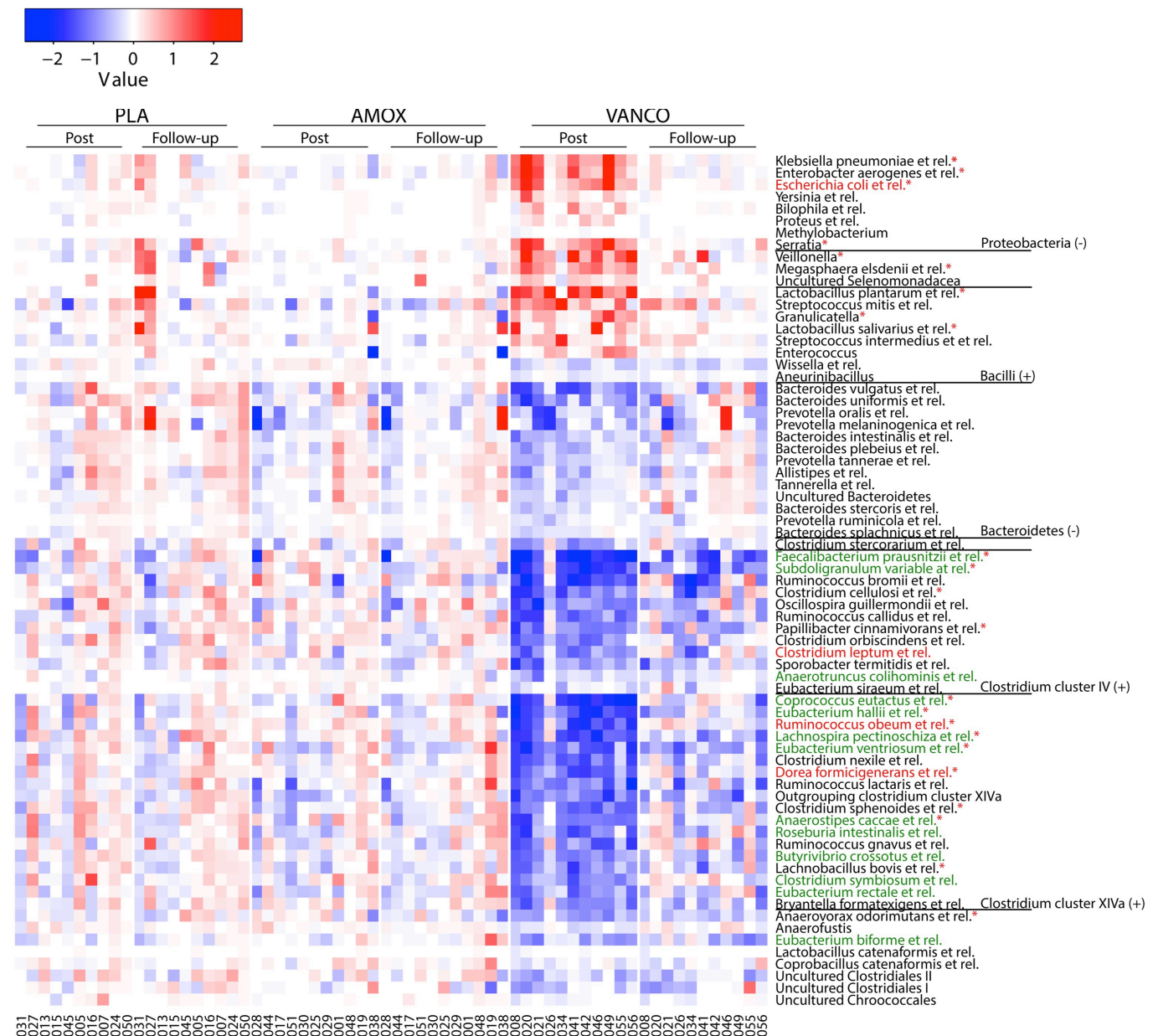

Figure 2.1. The effect of vancomycin and amoxicillin treatment on microbiota composition. Heatmap of bacterial groups (at genus and order like level with Gram staining between brackets) whose relative abundance was significantly different $(\mathrm{q}<0.05)$ post-treatment within the VANCO group. Color value shows $\log _{10}$ fold changes compared to baseline. Genus like groups containing known butyrate producing and BA dehydroxylating species are depicted in green and red, respectively. ${ }^{*}$ Groups that exhibited a significant difference between VANCO and PLA treatments. 


\section{Antibiotic treatment does not affect tissue-specific insulin sensitivity}

The primary outcome of this study was peripheral insulin sensitivity (insulinstimulated glucose rate of disappearance, $\mathrm{Rd}$ ), as determined by a two-step hyperinsulinemic-euglycemic clamp with $\left[6,6-{ }^{2} \mathrm{H}_{2}\right]$-glucose tracer infusion. Antibiotic treatment did not significantly alter Rd as compared to PLA (Figure 2.2). Additionally, no effects were found on hepatic and adipose tissue (AT) insulin sensitivity, as determined by the insulin-mediated suppression of endogenous glucose production (EGP) and plasma free fatty acid (FFA) concentrations, respectively. In accordance, antibiotic treatment did neither alter whole-body insulin sensitivity (HOMA-IR) immediately after cessation of treatment, nor at eight weeks follow-up (Figure S2). Our data are in contrast with several previous studies in rodents, which indicated that antibiotic treatment may improve glucose homeostasis and metabolic impairments ${ }^{14-17,21-23}$. Nevertheless, a more recent study showed that VANCO-treated mice had little weight change and no improvement in glycemic control ${ }^{24}$. Consistent with the present data, a four-day treatment with a broad-spectrum antibiotic cocktail did not affect postprandial glucose metabolism in lean healthy men ${ }^{25}$. Furthermore, it has recently been shown in a limited number of obese subjects with the metabolic syndrome that VANCO slightly but significantly reduced peripheral insulin sensitivity, despite comparable changes in microbial composition and BA metabolism as found in the present study ${ }^{18}$. Although the data of the latter study seems at odds with the present findings, it is important to emphasize that in the study by Vrieze and colleagues ${ }^{18}$ the modest $(\sim 4 \%)$ VANCO-induced decrease in peripheral insulin sensitivity was based on a within-group comparison (posttreatment versus pre-treatment), since a placebo group was not included in the study design. Additionally, in the present study, follow-up measurements that were performed eight weeks after treatment cessation also did not show an effect on whole-body insulin sensitivity, despite a still considerably altered microbial composition as compared to pre-treatment as well as placebo. 
A

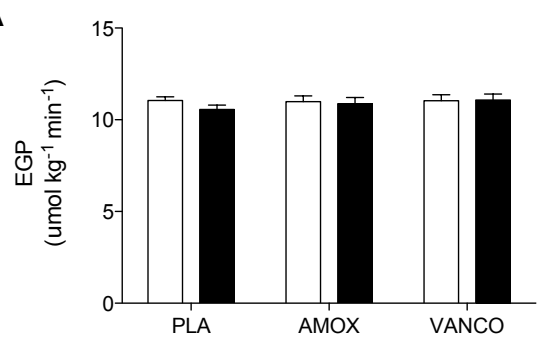

C

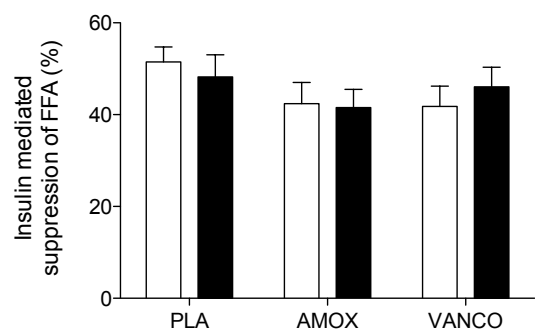

B

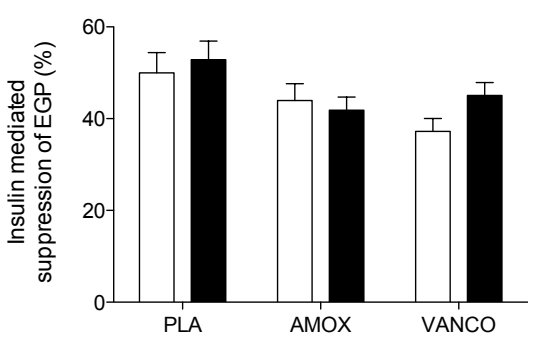

D

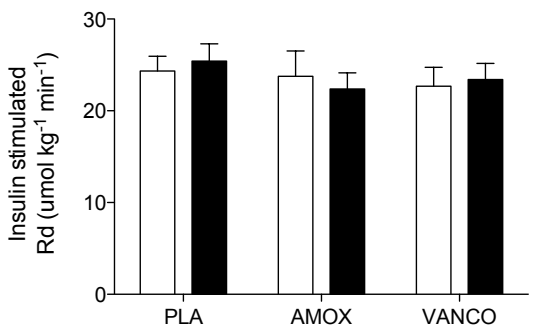

Figure 2.2. The effect of 7 days placebo, amoxicillin and vancomycin on hepatic, adipose tissue and peripheral insulin sensitivity. Bars represent means \pm SEM $(n=56)$. Tissue specific insulin sensitivity did not change by short-term antibiotics. (A) EGP: liver endogenous glucose production at baseline (B) steady state insulinmediated EGP suppression (\%) upon $10 \mathrm{mU} / \mathrm{m}^{2} / \mathrm{min}$ insulin infusion (C) steady state 10 $\mathrm{mU} / \mathrm{m}^{2} / \mathrm{min}$ insulin-mediated suppression (\%) of circulating free fatty acids (FFA) as measure for adipose tissue insulin sensitivity. (D) $40 \mathrm{mU} / \mathrm{m}^{2} / \mathrm{min}$ insulin stimulated (oxidative) glucose disposal (Rd).

\section{Antibiotic treatment does not affect energy and substrate metabolism}

To examine the effect of gut microbiota modulation on postprandial metabolite concentrations, energy expenditure and substrate oxidation, we performed a high-fat mixed-meal test (2.6MJ [61E\% fat, 33E\% carbohydrates, $6 \mathrm{E} \%$ protein]). We determined arterialized plasma metabolite concentrations and measured energy expenditure and substrate oxidation by whole-body indirect calorimetry. Neither VANCO nor AMOX significantly affected basal and postprandial plasma glucose, insulin, FFA, triacylglycerol (TAG) and lactate concentrations (Table 2.2 and Figure S2.3). Also, no significant effects on basal and postprandial energy expenditure, carbohydrate and fat oxidation were found (Figure 2.3). After 
adjustment for fecal weight, intestinal energy harvest, which is reflected by daily fecal energy content, was neither changed immediately after treatment cessation, nor after eight weeks follow-up (Figure 2.3). Although previous studies in rodents have shown a prominent role of the gut microbiota in energy harvest and body weight ${ }^{3,13}$, our findings suggest that antibiotics do not alter energy harvest in humans. Of note, in rodent studies, animals were exposed to antibiotics in their drinking water for two up to twenty weeks ${ }^{13-17,21-23}$. Similarly, more prolonged treatment (four to six weeks) with a higher dosage or a combination of different antibiotics increased body weight in endocarditis patients ${ }^{26,27}$. These studies may indicate that a long-term dysbalance in microbiota composition has more pronounced effects as compared to short-term manipulation. However, it is hard to differentiate between the role of the gut microbiota and systemic effects of antibiotics in the latter studies. Noteworthy, we have applied a two-day wash-out period before post-treatment measurements were performed to exclude that effects may be mediated via direct systemic effects of antibiotics. Additionally, VANCO does not pass the gastrointestinal barrier and, therefore, does not reach the circulation ${ }^{28}$.

\section{Antibiotic treatment does not alter gut permeability and systemic inflammatory markers}

We investigated the effect of seven days of AMOX and VANCO treatment on gut permeability and the related translocation of bacterial lipopolysaccharide (LPS) from the intestinal lumen into the circulation. The pronounced VANCO-induced microbial alterations were not accompanied by changes in small intestine and proximal colon permeability (Figure S2.4), as assessed by a multi-saccharide test $^{29}$. This is in accordance with unchanged LPS-binding protein (LBP) concentrations after VANCO and AMOX treatment as compared to PLA (Table 2.2). LPS, which is released by Gram-negative bacteria, may trigger the immune system by increasing inflammatory cytokine production in AT and is frequently used as an indicator of metabolic endotoxemia ${ }^{30}$. Therefore, we have additionally determined plasma interleukin (IL)-6, IL-8 and tumor necrosis factor (TNF)- $\alpha$ concentrations. In line with unchanged LBP concentrations, neither of these inflammatory factors was affected by seven-day VANCO or AMOX as compared to PLA. This was observed despite a substantial increase in relative abundance of potentially pro-inflammatory Gram-negative Proteobacteria. 
Table 2.2. Effect of 7 days placebo, amoxicillin and vancomycin on metabolic, inflammatory, and hormonal parameters

\begin{tabular}{|c|c|c|c|c|c|}
\hline \multicolumn{2}{|c|}{ Variable } & PLA $(\mathrm{N}=14)$ & $\operatorname{AMOX}(\mathrm{N}=12)$ & VANCO $(\mathrm{N}=12)$ & Pvalue $^{\mathrm{a}}$ \\
\hline \multicolumn{6}{|c|}{ Fasting plasma metabolite and ANGPTL4 concentrations } \\
\hline \multirow[t]{2}{*}{ Glucose (mM) } & Pre & $6.31 \pm 1.12$ & $6.48 \pm 0.25$ & $6.25 \pm 0.19$ & $0.177^{\mathrm{b}}$ \\
\hline & Post & $6.29 \pm 0.14$ & $6.39 \pm 0.20$ & $5.99 \pm 0.13$ & \\
\hline \multirow[t]{2}{*}{ TAG (mM) } & Pre & $1.40 \pm 0.17$ & $1.08 \pm 0.15$ & $1.03 \pm 0.09$ & 0.511 \\
\hline & Post & $1.47 \pm 0.21$ & $1.04 \pm 0.15$ & $1.06 \pm 0.10$ & \\
\hline \multirow[t]{2}{*}{ FFA (uM) } & Pre & $699 \pm 34$ & $683 \pm 48$ & $679 \pm 38$ & $0.423^{b}$ \\
\hline & Post & $661 \pm 34$ & $579 \pm 58$ & $626 \pm 54$ & \\
\hline \multirow[t]{2}{*}{ Lactate (mM) } & Pre & $0.80 \pm 0.07$ & $0.93 \pm 0.04$ & $0.88 \pm 0.11$ & 0.238 \\
\hline & Post & $0.91 \pm 0.11$ & $0.90 \pm 0.05$ & $0.79 \pm 0.06$ & \\
\hline \multirow[t]{2}{*}{$\begin{array}{l}\text { ANGPTL4 } \\
(\mathrm{ng} / \mathrm{ml})\end{array}$} & Pre & $5.1 \pm 0.7$ & $4.3 \pm 0.5$ & $4.9 \pm 0.5$ & 0.137 \\
\hline & Post & $5.5 \pm 0.7$ & $3.8 \pm 0.5$ & $4.3 \pm 0.3$ & \\
\hline \multicolumn{6}{|c|}{ Postprandial (0-4h) plasma metabolite concentrations } \\
\hline \multirow[t]{2}{*}{$\begin{array}{l}\text { Glucose } \\
\text { (iAUC/min) }\end{array}$} & Pre & $0.60 \pm 0.10$ & $0.50 \pm 0.12$ & $0.41 \pm 0.19$ & 0.633 \\
\hline & Post & $0.54 \pm 0.13$ & $0.55 \pm 0.10$ & $0.45 \pm 0.09$ & \\
\hline \multirow[t]{2}{*}{ TAG (iAUC/min) } & Pre & $0.84 \pm 0.07$ & $0.83 \pm 0.10$ & $0.75 \pm 0.10$ & $0.945^{b}$ \\
\hline & Post & $0.92 \pm 0.07$ & $0.94 \pm 0.01$ & $0.81 \pm 0.07$ & \\
\hline \multirow[t]{2}{*}{$\begin{array}{l}\text { FFA } \\
\text { (iAUC/min) }\end{array}$} & Pre & $-364 \pm 27$ & $-341 \pm 36$ & $-339 \pm 31$ & $0.547^{b}$ \\
\hline & Post & $-332 \pm 28$ & $-245 \pm 37$ & $-300 \pm 40$ & \\
\hline \multirow[t]{2}{*}{$\begin{array}{l}\text { Lactate } \\
\text { (iAUC/min) }\end{array}$} & Pre & $0.65 \pm 0.05$ & $0.45 \pm 0.07$ & $0.40 \pm 0.08$ & $0.154^{b}$ \\
\hline & Post & $0.50 \pm 0.05$ & $0.41 \pm 0.06$ & $0.41 \pm 0.06$ & \\
\hline \multicolumn{6}{|c|}{ Fasting plasma hormone concentrations } \\
\hline \multirow[t]{2}{*}{ GLP-1 (pmol/l) } & Pre & $8.7 \pm 0.7$ & $8.5 \pm 0.7$ & $9.7 \pm 1.1$ & 0.670 \\
\hline & Post & $9 \cdot 3 \pm 1.1$ & $8.7 \pm 0.8$ & $10.2 \pm 1.2$ & \\
\hline \multirow[t]{2}{*}{ Insulin (mU/l) } & Pre & $11.5 \pm 1.3$ & $12.6 \pm 1.3$ & $14 \cdot 3 \pm 1.8$ & 0.504 \\
\hline & Post & $12.7 \pm 1.6$ & $13.4 \pm 1.8$ & $13.9 \pm 1.5$ & \\
\hline \multirow[t]{2}{*}{ Leptin (ng/ml) } & Pre & $11.4 \pm 1.6$ & $10.1 \pm 2.1$ & $9.7 \pm 0.8$ & $0.106^{b}$ \\
\hline & Post & $12.9 \pm 2.3$ & $10.0 \pm 1.8$ & $8.8 \pm 0.8$ & \\
\hline \multicolumn{6}{|c|}{ Postprandial (o-4h) plasma hormone concentrations } \\
\hline \multirow[t]{2}{*}{$\begin{array}{l}\text { GLP-1 } \\
\text { (iAUC/min) }\end{array}$} & Pre & $5.0 \pm 0.7$ & $4.3 \pm 0.7$ & $3.4 \pm 0.6$ & 0.451 \\
\hline & Post & $4.5 \pm 0.8$ & $4 \cdot 3 \pm 0.8$ & $4.3 \pm 0.9$ & \\
\hline \multirow[t]{2}{*}{$\begin{array}{l}\text { Insulin } \\
\text { (iAUC/min) }\end{array}$} & Pre & $21.1 \pm 2.1$ & $20.0 \pm 3.0$ & $25.0 \pm 4.5$ & 0.294 \\
\hline & Post & $23.1 \pm 3.3$ & $21.7 \pm 3.0$ & $22.5 \pm 3.0$ & \\
\hline
\end{tabular}




\begin{tabular}{llllll}
\hline Variable & \multicolumn{1}{c}{ PLA (N=19) } & AMOX (N=18) & VANCO (N=19) & Pvalue $^{\text {a }^{2}}$ \\
\hline \multicolumn{2}{l}{ Fasting inflammatory marker concentrations } & & & \\
LBP $(\mathrm{pg} / \mathrm{ml})$ & Pre & $19.6 \pm 1.8$ & $17.5 \pm 1.8$ & $25.7 \pm 4.3$ & 0.456 \\
& Post & $18.4 \pm 3.3$ & $20.4 \pm 2.9$ & $23.6 \pm 3.6$ & \\
$\mathrm{IL}-6(\mathrm{pg} / \mathrm{ml})$ & Pre & $0.8 \pm 0.1$ & $0.8 \pm 0.1$ & $1.0 \pm 0.1$ & 0.775 \\
& Post & $1.0 \pm 0.1$ & $0.8 \pm 0.1$ & $1.1 \pm 0.1$ & \\
$\mathrm{IL}-8(\mathrm{pg} / \mathrm{ml})$ & Pre & $6.2 \pm 0.5$ & $4 \cdot 3 \pm 0.4^{c}$ & $5.2 \pm 0.4$ & 0.444 \\
& Post & $5.9 \pm 0.5$ & $4.8 \pm 0.4$ & $5.9 \pm 0.4$ & \\
TNF- $\alpha(\mathrm{pg} / \mathrm{ml})$ & Pre & $2.6 \pm 0.1$ & $2.3 \pm 0.1^{\mathrm{c}}$ & $2.7 \pm 0.1$ & 0.424 \\
& Post & $2.7 \pm 0.1$ & $2.5 \pm 0.1$ & $2.8 \pm 0.1$ & \\
\hline
\end{tabular}

Data are mean \pm SEM. For determination of plasma hormones and metabolites, only a subgroup of $n=38$ was analyzed. There were no significant differences between the groups after intervention (Post) compared to baseline (Pre). Triacylglycerol (TAG), free fatty acids (FFA), glucagon-like peptide (GLP), angiopoietin-like 4 (ANGPTL4), lipopolysaccharide-binding protein (LBP), interleukin (IL), tumor necrosis factor (TNF). ${ }^{a} \mathrm{P}$ value represents the overall intervention effect between groups assessed by repeated measures ANOVA (timextreat $P$ value) or ANCOVA when baseline concentrations were different between groups. ${ }^{b}$ time effect $(P<0.05) .{ }^{C}$ baseline group difference $(P<0.05)$

\section{Vancomycin inhibits bile acid conversion and short-chain fatty acid production}

SCFA, notably butyrate, can be produced by several groups within the Firmicutes phylum (mainly Clostridium clusters XIVa and IV including Coprococcus eutactus and $F$. prausnitzii), some of which are also involved in BA dehydroxylation ${ }^{9,31}$. Indeed, we found a decreased relative abundance of these groups after VANCO, which was accompanied by a marked reduction in plasma $(P=0.005)$ and fecal $(P=0.001)$ concentrations of secondary BA as compared to PLA (Figure 2.4). This was accompanied by an increase of fecal primary $B A(P=0.013)$. In addition, fecal SCFA concentrations (acetate $(P=0.001)$, butyrate $(P<0.001)$, caproate $(P<0.001)$ and valerate $(P=0.009)$ ) were significantly decreased following VANCO, whilst in plasma only butyrate tended to decrease after VANCO $(P=0.078)$ but not following AMOX treatment (Figure 2.5).

Although BA and SCFA may control incretin release ${ }^{10,32}$ and affect energy metabolism in rodents ${ }^{33}$, no effects on postprandial energy and substrate metabolism, and fasting and postprandial glucagon-like peptide 1 (GLP-1) concentrations were found in the present study (Table 2.2). 
- - Placebo Pre $\quad \because$ Amox Pre $\quad \nabla-$ Vanco Pre

- Placebo Post $\rightarrow$ Amox Post $\rightarrow$ Vanco Post

A

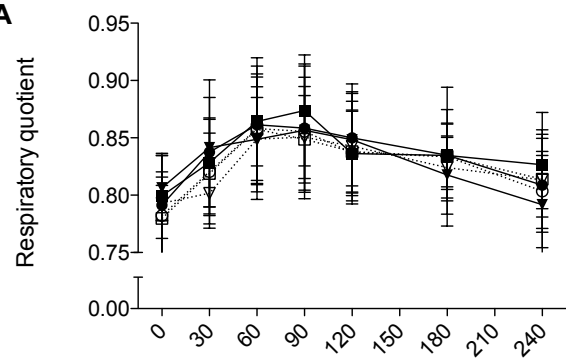

C
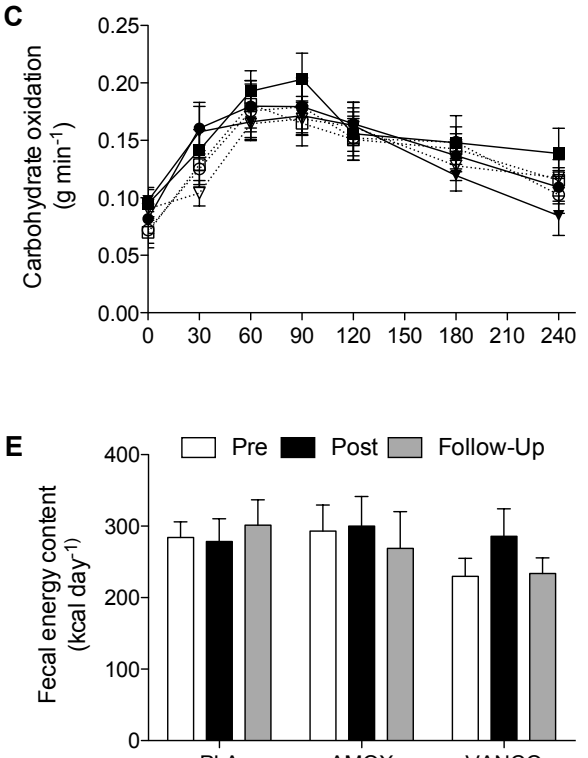

B

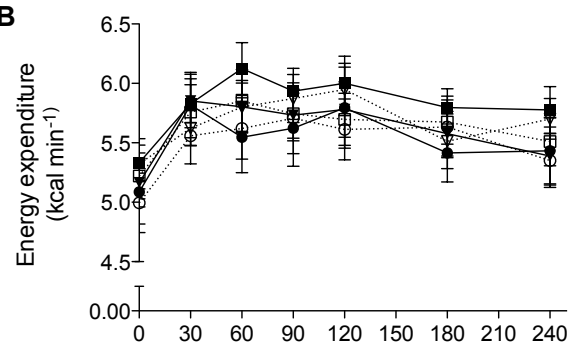

D

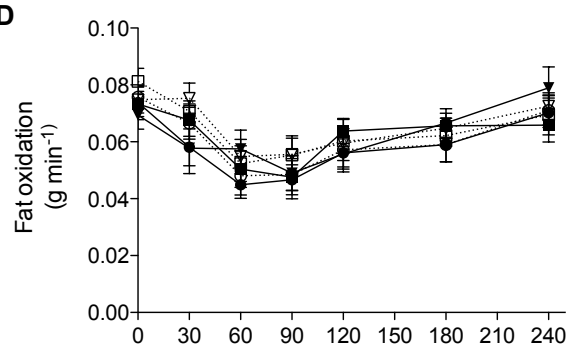

Figure 2.3. The effect of 7 days placebo, amoxicillin and vancomycin on energy expenditure, substrate metabolism and fecal energy excretion. Data are given as means \pm SEM. Indirect calorimetry was performed during fasting conditions and for 4 hours after intake of a liquid high fat mixed meal (HFMM) in a subgroup of $n=37$. Mean $\mathrm{O}_{2}$-consumption and $\mathrm{CO}_{2}$-production over 20 minutes were used for calculations. (A-D) Fasting respiratory quotient (RQ), energy expenditure (EE), carbohydrate oxidation and fat oxidation did not differ after intervention (timextreat $P$ value $>0.05$ ). Incremental AUC's after ingestion of HFMM were also not affected by AMOX or VANCO. (E) Fecal Energy excretion (kcal/day) did not significantly change after VANCO or AMOX compared to PLA $(n=56)$. 


\section{Antibiotic treatment alters adipose tissue gene expression but not adipocyte morphology.}

To determine the effect of an altered gut microbiota composition on AT, we collected abdominal subcutaneous AT biopsies to examine adipocyte size and gene expression profiles using Affymetrix microarray transcriptomic analysis. Antibiotic treatment had no significant effect on abdominal subcutaneous adipocyte size and the proportion of small and large adipocytes, neither directly after treatment cessation nor at eight weeks follow-up (Figure S2.5). Remarkably, when comparing the gene expression data with the Kyoto Encyclopedia of Genes and Genomes (KEGG), we found that VANCO and, to a lesser extent, AMOX increased AT expression of genes involved in pathways related to peroxisomeproliferator activated receptor (PPAR)-signaling and of genes encoding proteins involved in the mitochondrial Krebs cycle, fatty acid degradation and other components of the oxidative machinery, suggestive of increased oxidative metabolism in AT (Figure S2.6). In addition, VANCO decreased the expression of histone clustering genes. Although we found no differences in adipocyte morphology and circulating FFA, TAG, leptin and angiopoietin-like 4 (ANGPTL4) concentrations (Table 2.2), these alterations in the AT transcriptome may translate into changes in AT function over longer periods of time.

Finally, VANCO decreased the expression of gene sets involved in apoptosis and nuclear factor NFkB signaling as well as adaptive and innate immune responses, including genes of major histocompatibility complex-I, T-cell, B-cell and Natural Killer cell signaling. In contrast, genes related to lysosomal breakdown were upregulated as compared to PLA (Table S2.3). Lower NFkB-dependent gene expression and diminished $\mathrm{NK}$ and $\mathrm{CD} 8+\mathrm{T}$ cell function in macrophages have been observed in germ-free and antibiotic-treated mice ${ }^{34}$. In the latter study, the effects were ascribed to a reduced activation of Farnesoid $X$ receptors by a reduction of unconjugated and secondary $\mathrm{BA}^{9}$, which seems in line with the present findings. In addition, although the exact role of SCFA in the systemic and AT immune cell responses is unknown, SCFA may be involved in the regulation of $\mathrm{T}$-cells in the gut and peripheral tissues via the G-protein coupled receptor $43^{10,35-}$ 37. Despite the effects of antibiotic treatment on the KEGG-pathways described above, no significant associations (FDR<0.25) were found between individual bacterial groups and AT gene expression (data not shown). 

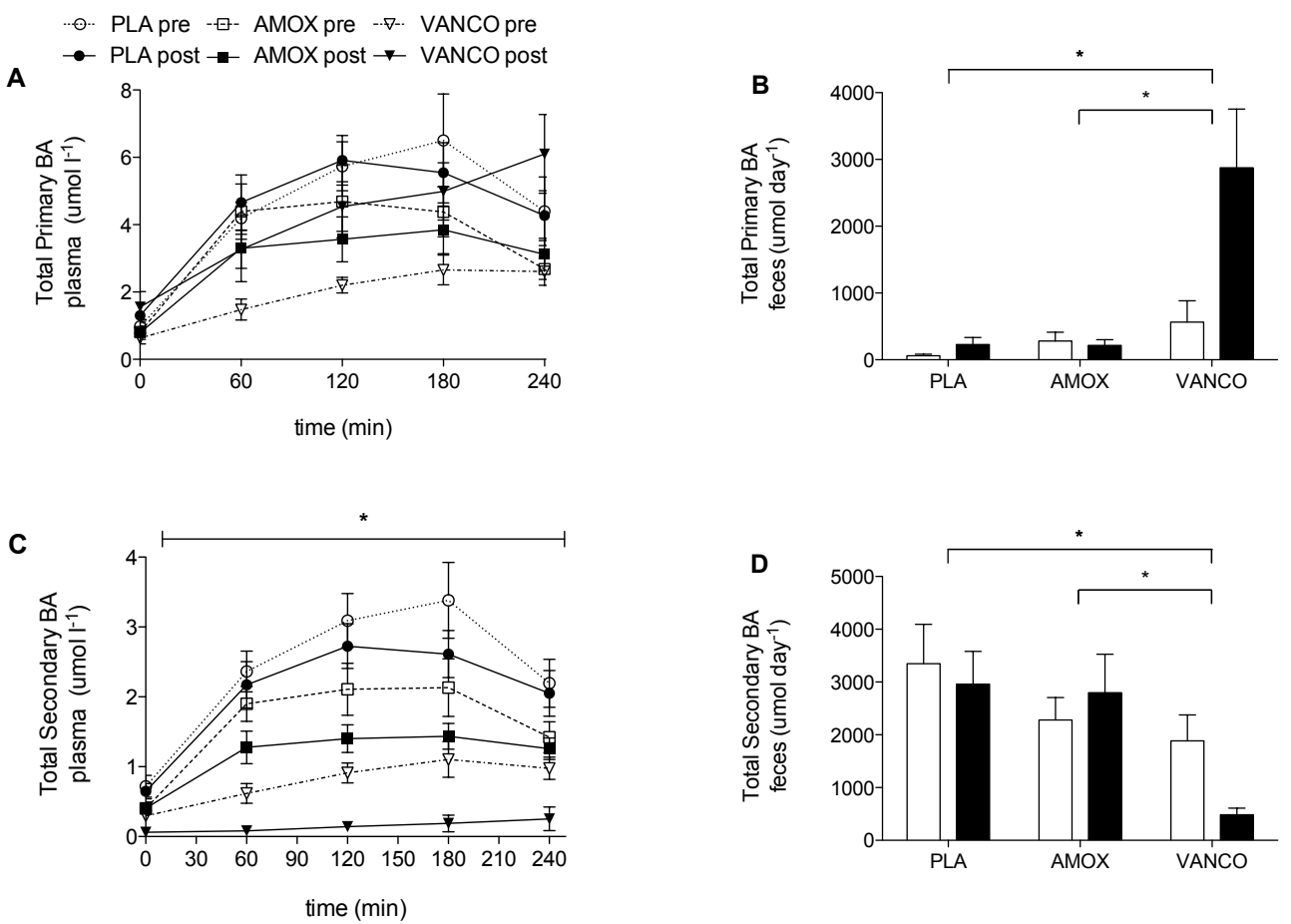

Figure 2.4. The effect of 7 days placebo, amoxicillin and vancomycin on bile acid concentrations in plasma and feces. Means $\pm S E M$. In a subgroup of $n=37,(A)$ plasma postprandial primary BA did not change significantly, (B) VANCO increased fecal primary $B A,(C)$ decreased plasma secondary $B A$ and $(D)$ fecal secondary BA compared to PLA and AMOX. * timextreat P-value $<0.05$ for VANCO vs. PLA.

\section{Microbial groups are not associated with host metabolic parameters}

Although overall host metabolism did not change significantly following antibiotic treatment, we used univariate and multivariate statistics (redundancy analysis) to assess possible associations between specific characteristics of gut microbial profiles and host metabolic parameters. However, we did not find any significant and consistent associations when we evaluated the abundance and dynamics of individual bacterial taxa, combinations of taxa, the complete microbiota and bacterial diversity at baseline, as well as seven days and eight weeks post-intervention. 
Furthermore, we investigated whether we could identify and connect patterns of specific metabolic and/or microbiological perturbations with the response to the intervention. First, we evaluated the stratification of subjects based on the extent of the microbial shift in diversity, as well as microbial composition. Secondly, based on the extent and direction of the metabolic response to the intervention, we used univariate and cluster analysis to discover microbial patterns. Lastly, we used latent class analysis ${ }^{38}$, to define groups of subjects with certain metabolic patterns before and after treatment. Neither of these analyses showed groups of individuals with specific associations of the microbiota with host metabolic parameters (data not shown).
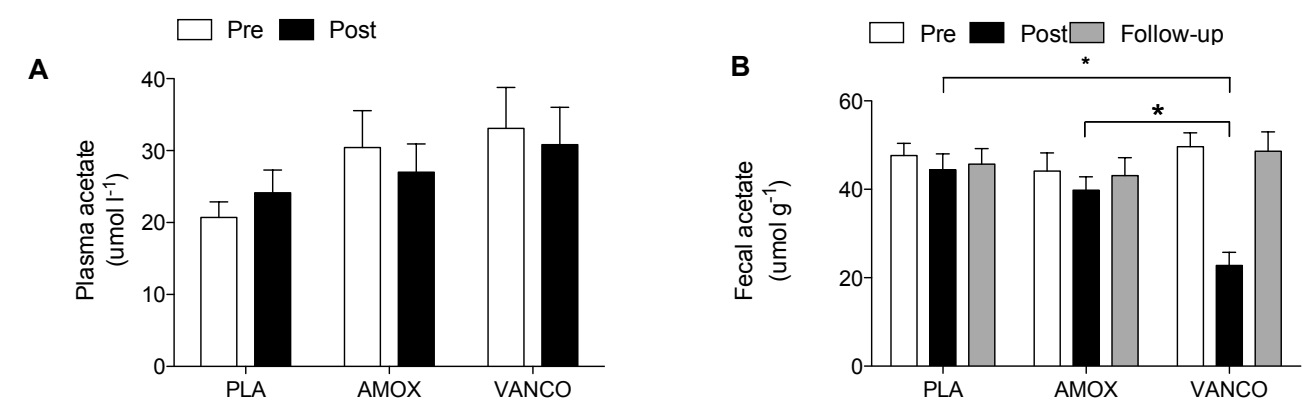

C

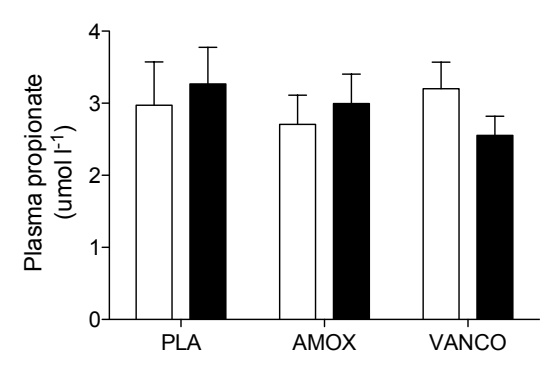

D
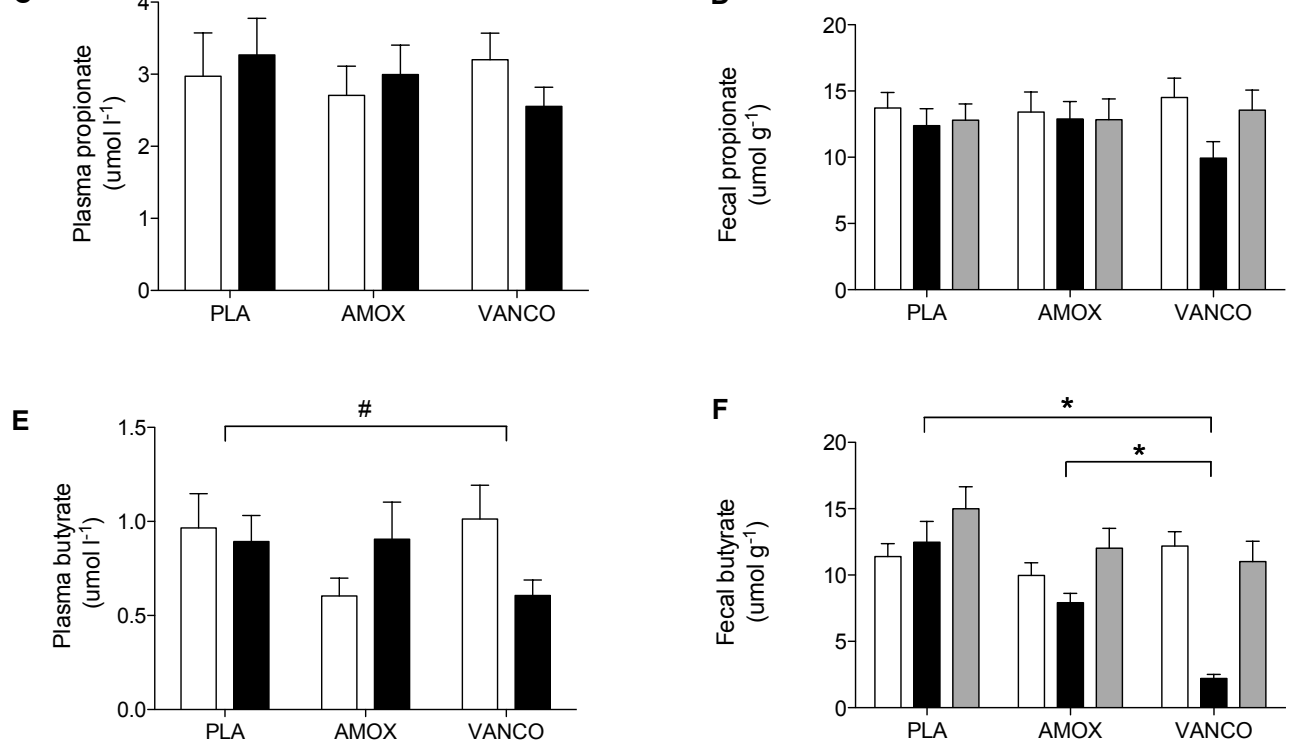
Figure 2.5: The effect of 7 days placebo, amoxicillin and vancomycin on plasma and fecal short chain fatty acid concentrations. Values are given as mean \pm SEM $(n=56)$. No significant effect was found for (A) plasma acetate, (C) plasma propionate, $(D)$ fecal propionate, and a trend $(\# P=0.07)$ for $(E)$ plasma butyrate. Fecal acetate and butyrate $(B, F)$ decreased after VANCO treatment but not after AMOX. * timextreat $P$-value $<0.05$ for VANCO vs. PLA and vs. AMOX.

\section{Perspectives}

In the present study, we demonstrated that seven days VANCO treatment markedly affected microbial diversity and composition, which was accompanied by a reduced conversion of primary to secondary BA and a lower production of SCFA in the gut. Importantly, these alterations did not translate into significant effects on peripheral, hepatic and AT insulin sensitivity, energy and substrate metabolism and systemic low-grade inflammation immediately after treatment cessation. Moreover, no clinically relevant effects on energy harvest, abdominal subcutaneous adipocyte size and whole-body insulin sensitivity (HOMA-IR) were found at eight weeks follow-up. In contrast to VANCO, no effects of AMOX treatment on gut microbial composition, metabolic and inflammatory parameters were found. Taken together, the present study implies that interference with a resilient adult microbiota by antibiotics has no clinically relevant short-term (seven days) and long-term (eight weeks) effects on the metabolic parameters measured in this study. This contradicts many previous rodents studies and again highlights that rodent data cannot always be extrapolated to humans.

Noteworthy, several nuances have to be made with respect to the conclusions of the present study. First, since we studied obese, insulin resistant men with impaired glucose metabolism, we cannot exclude that microbiota manipulation by antibiotics may have more pronounced effects in women or less metabolically compromised individuals. Secondly, the duration of the intervention was relatively short, compared to rodent studies. Furthermore, it has been demonstrated that the risk of developing type 2 diabetes was increased when subjects were exposed to $>5$ antibiotic treatments ${ }^{39}$, and that the number of prescriptions may accelerate the ageing-related decline of intestinal integrity ${ }^{40}$. Of note, the participants that were included in the present study had received on average 1.7 antibiotic treatments over the past 10 years, without any antibiotic 
use 3 months prior to the start of the study. As mentioned above, several studies have indicated that a long-term or more frequent perturbation in microbiota composition may have more pronounced effects on metabolic health than shortterm manipulation. For this reason, it is important to emphasize that the present study does not exclude an important role for the gut microbiota manipulations in changes of host metabolism. This should be further investigated in future prospective and long-term (dietary, prebiotic and/or probiotic) intervention studies in humans. 
Supplementary Figures and Tables 

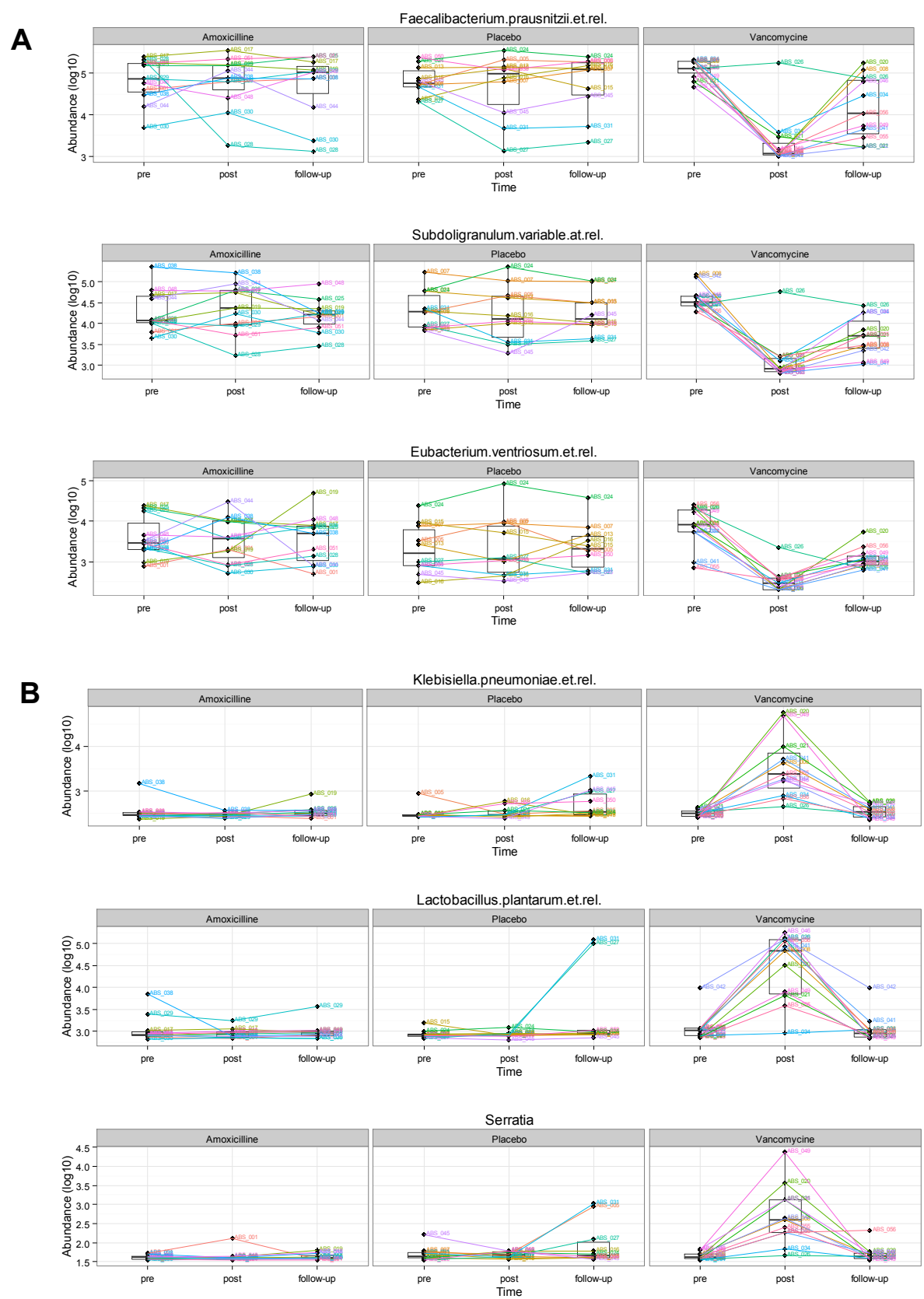

Figure S2.1. The effect of vancomycin, amoxicillin and placebo treatment on gut microbiota composition before, 7 days after and 8 weeks after intervention. Vancomycin treatment had highly pervasive effects on decreasing (A) and increasing (B) bacterial abundance, diversity (C) and overall similarity (D) as compared to baseline and placebo. Different individuals are indicated with different colours. 

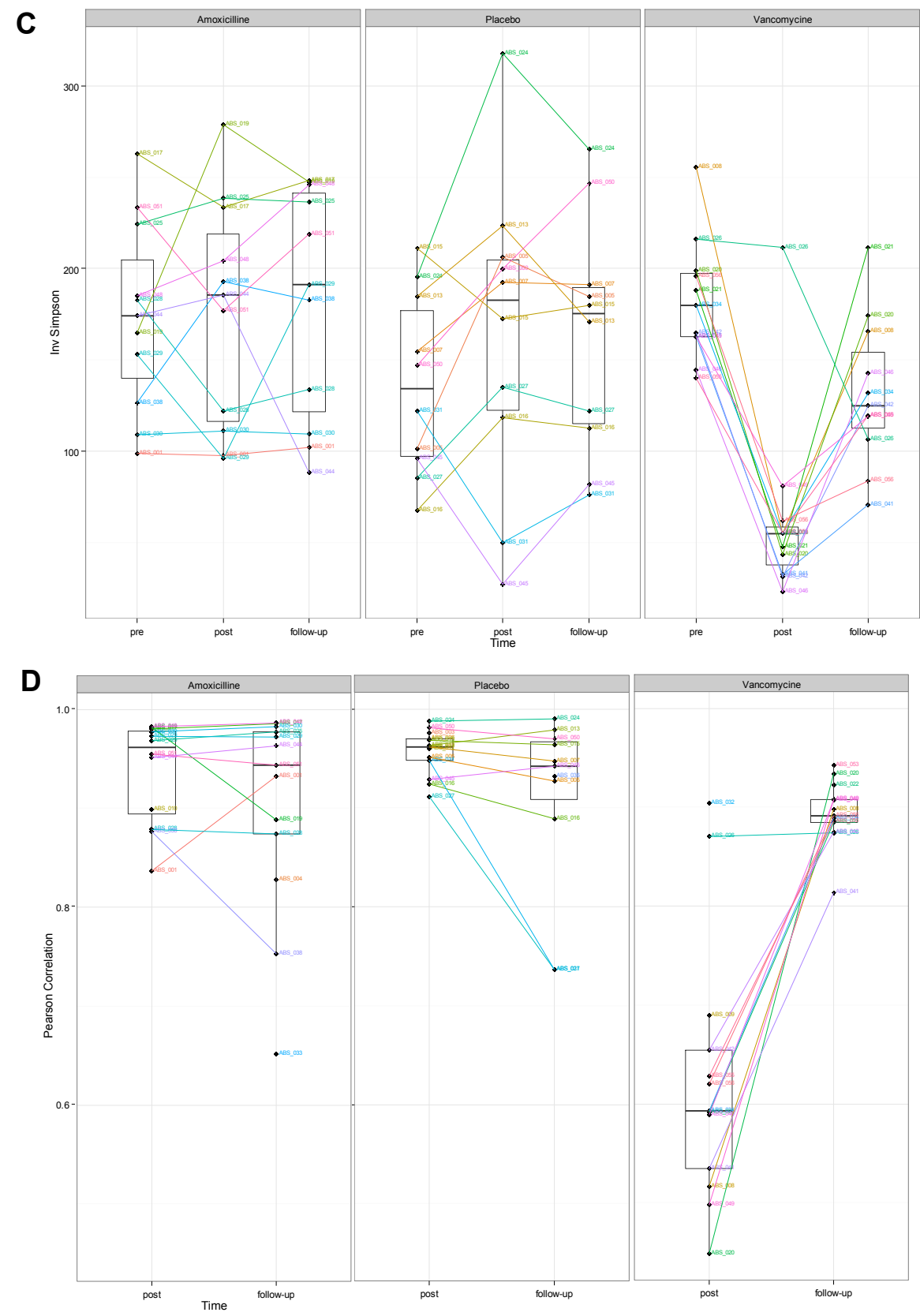

Although the abundance of most Gram-negative and vancomycin-resistant bacteria returned to near pre-treatment (baseline) levels at follow-up, some Gram-positive bacteria had not recovered in abundance in some individuals. A similar trend was observed for both diversity and compositional similarity at follow-up compared to baseline. 


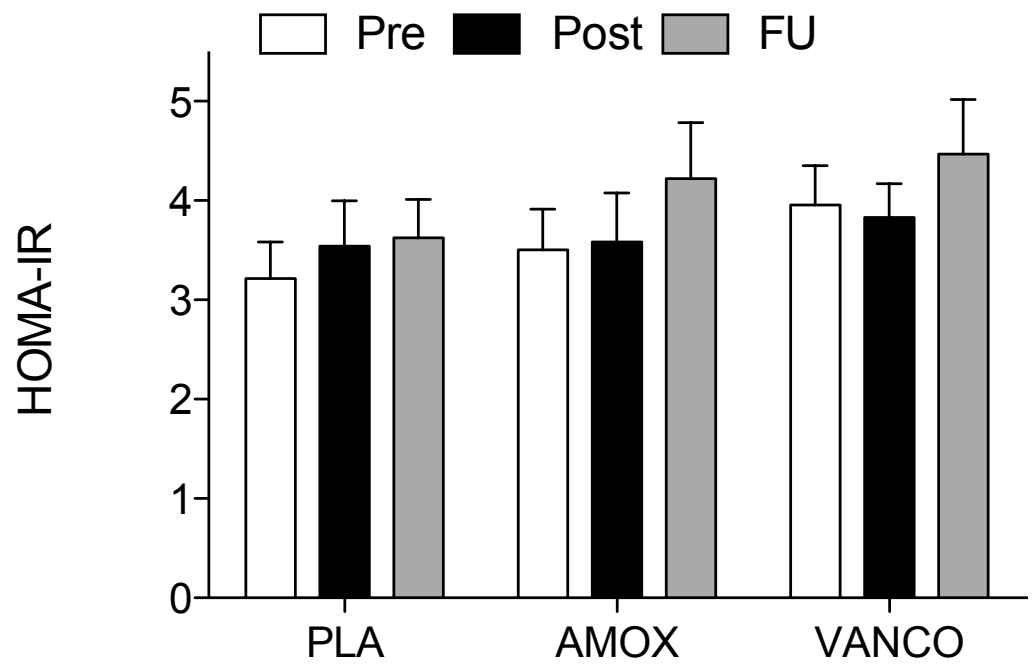

Figure S2.2. The effect of vancomycin, amoxicillin and placebo treatment for 7 days on whole-body insulin sensitivity directly after cessation of treatment and at 8-weeks follow-up. Data are mean \pm SEM $(n=37)$. AMOX and VANCO did not affect whole-body insulin sensitivity, reflected by the homeostasis model assessment of insulin resistance (HOMA-IR). There was a trend for a time-effect, but no timextreatment effect for FU compared to baseline ( $P=0.078)$. $P R E$, baseline values; POST, values directly after treatment cessation; $\mathrm{FU}$, values at 8-weeks follow-up. 


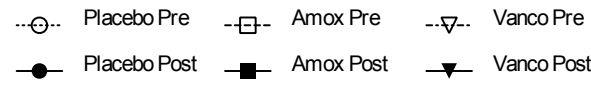

A

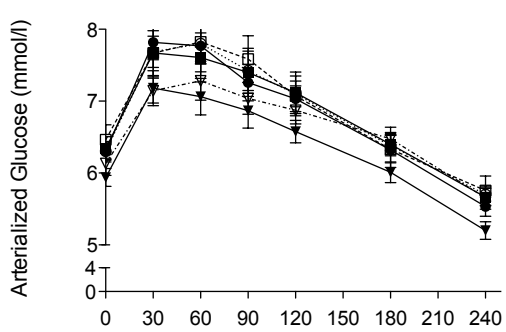

C

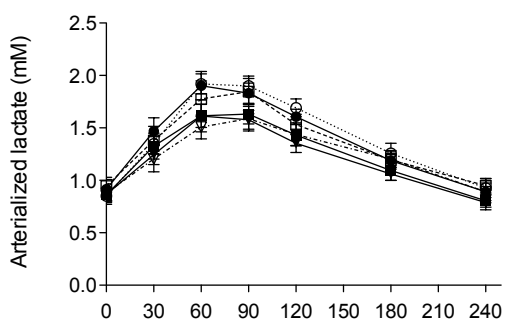

E

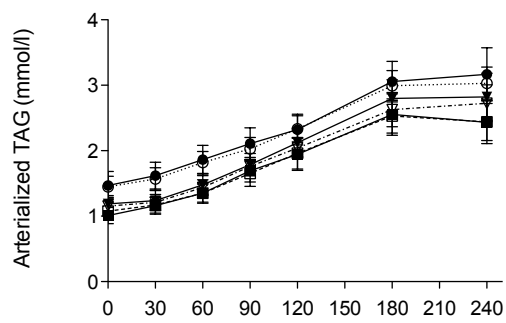

B
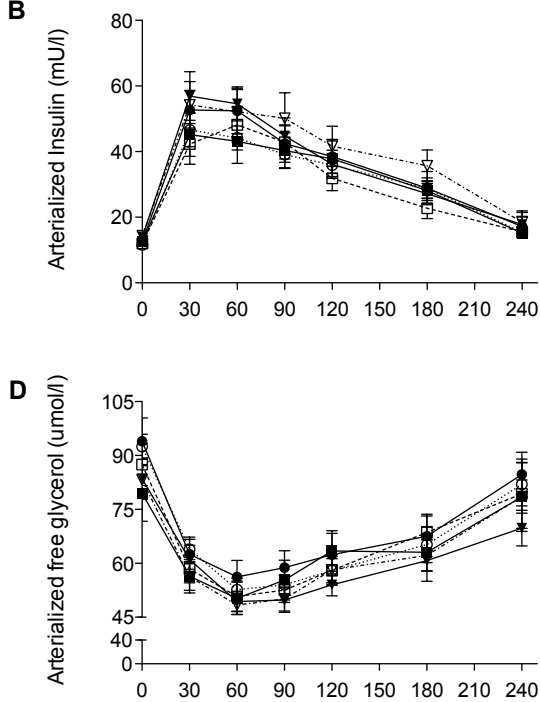

$\mathbf{F}$

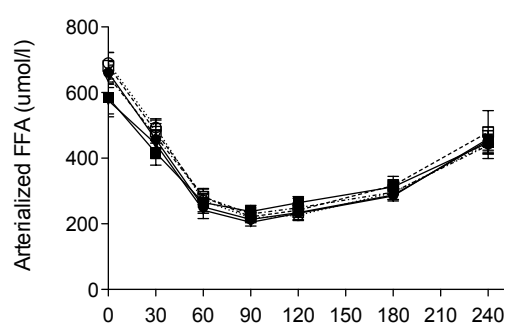

Figure S2.3. The effect of vancomycin, amoxicillin and placebo treatment for 7 days on plasma metabolite concentrations. Data are mean $\pm \operatorname{SEM}(n=37)$. Fasting $(t=0)$ and postprandial concentrations of $(A)$ arterialized glucose, $(B)$ lactate, (C) free glycerol, (D) triacylglycerol (TAG) and (E) free fatty acid (FFA) concentrations were not affected by AMOX or VANCO as assessed by repeated measures ANOVA. 

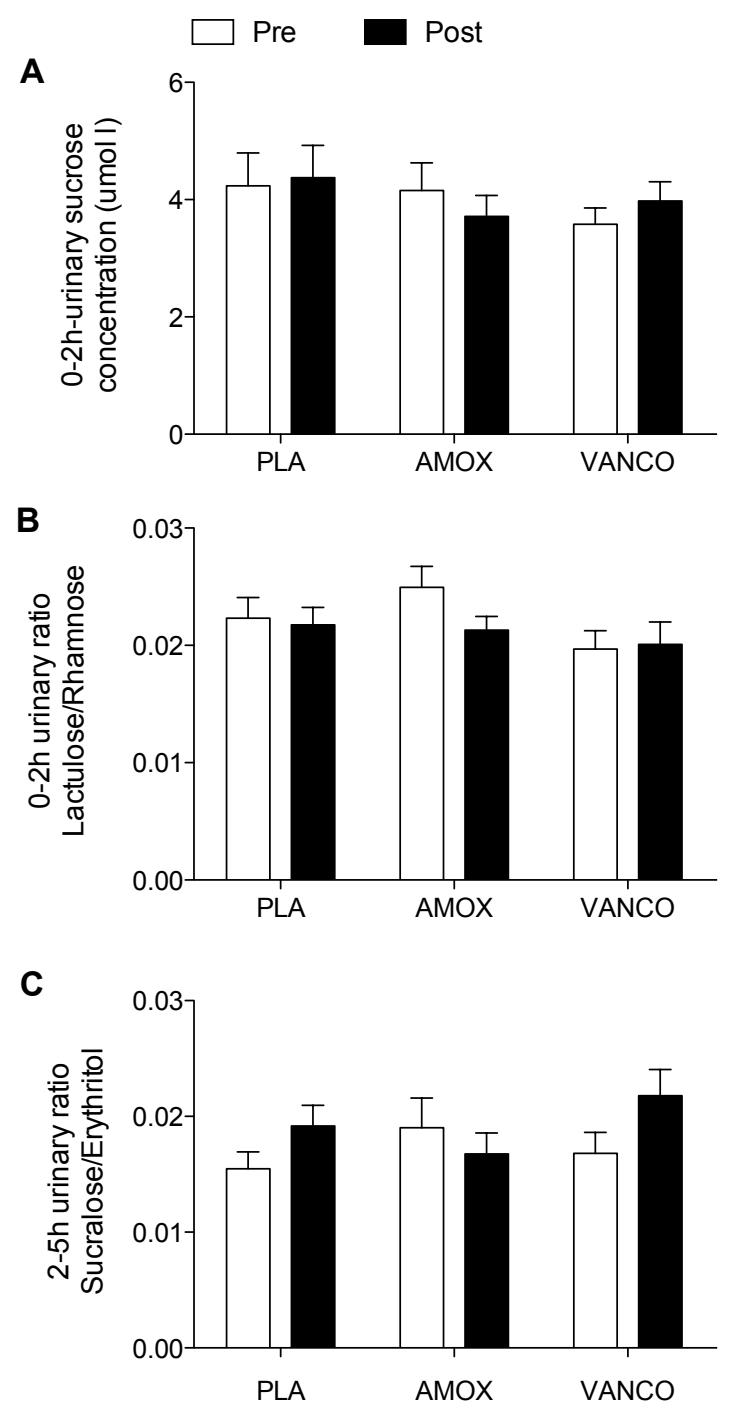

Figure S2.4. The effect of vancomycin, amoxicillin and placebo treatment for 7 days on gut permeability. Bars represent means $\pm \operatorname{SEM}(n=56)$. To determine gut permability, urine was collected immediately before drinking a multisaccharide-mix (to), at $t=120$ and $t=300$ minutes. AMOX and VANCO did not affect (A) gastroduodenal permeability as expressed by the urinary sucrose concentration at $t=120 \mathrm{~min}$, (B) small interstine permeability expressed by the lactulose/rhamnose ratio (urine collection 0-120 $\mathrm{min}$ ), (C) proximal colon permeability: suclarose/erythritol ratio (urine collection 120-300 $\min )$. 
A

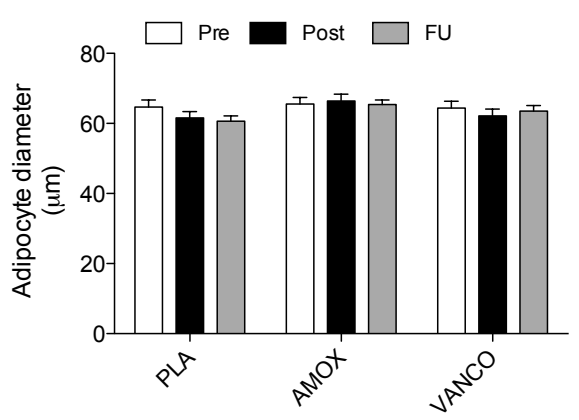

C

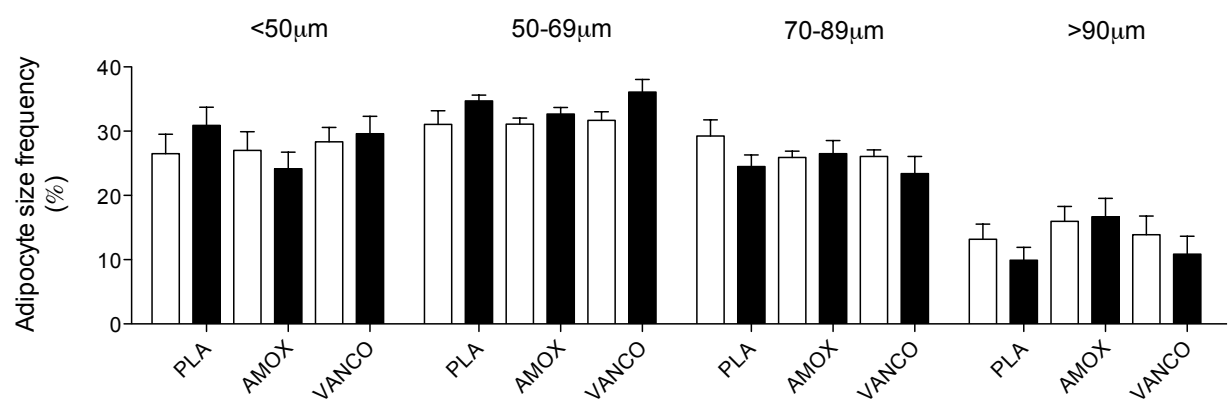

Figure S2.5. Abdominal subcutaneous adipocyte morphology before and immediately after placebo, amoxicillin and vancomycin treatment, as well as after $\mathbf{8}$ weeks follow-up. Representative sections of stained adipose tissue were used for adipocyte size determination in a subgroup of subjects $(n=18)$. AMOX and VANCO did not affect (A) mean adipocyte diameter, (B) mean adipocyte surface area and (C) adipocyte size distribution. 


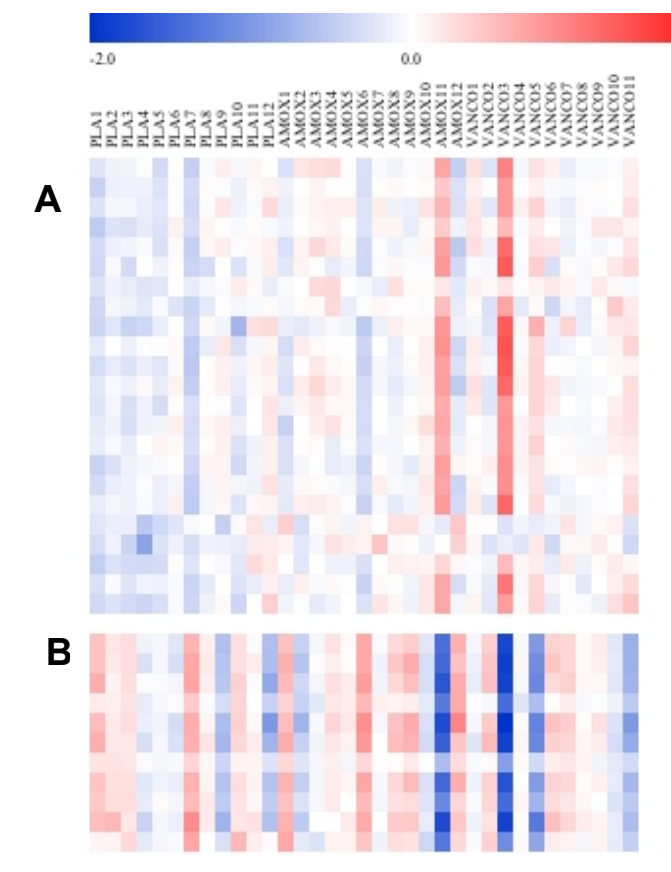

CITRATE.CYCLE.TCA.CYCLE

GLYCOLYSIS.GLUCONEOGENESIS

OXIDATIVE.PHOSPHORYLATION

GALACTOSE.METABOLISM

PYRUVATE.METABOLISM

RETINOL.METABOLISM

STARCH.SUCROSE

ARACHIDONIC ACID METABOLISM

ETHER.LIPID METABOLISM

FA.OXIDATION

PPAR

PROPANOATE METABOLISM

CYSTEINE AND METHIONINE METABOLISM

GLYCINE. SERINE.AND. THREONINE.METABOLISM

GLYCINE.SERINE.AND.THR

LYSINE.DEGRADATION
PROTEIN.DIGESTION.AND.ABSORPTION

PROTEIN.DIGESTION.AND

TYROSINE.METABOLISM
VALINE.LEUCINE.AND.ISOLEUCINE.DEGRADATION

ANTIGEN.PROCESSING.AND.PRESENTATION

INTESTINAL.IMMUNE.NETWORK.FOR.IGA.PRODUCTION

LYSOSOME

DRUG.METABOLISM.CYTOCHROME.P450

MINERAL.ABSORPTION

NATURAL.KILLER.CELL.MEDLATED.CYTOTOXICITY

B.CELL.RECEPTOR.SIGNALING.PATHWAY

T.CELL.RECEPTOR.SIGNALING.PATHWAY

ALLOGRAFT REJECTION

PRIMARY IMMUNODEFICIENCY

HEMATOPOETIC.CELL.LINEAGE

APOPTOSIS

NFkappaB

TNF

NOD

SYSTEMIC.LUPUS.ERYTHEMATOSUS (=HISTONE CLUSTERING)

\section{Upregulated pathways after vancomycin treatment}

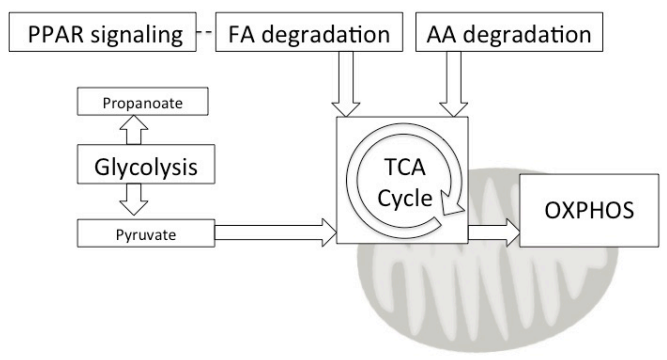

Downregulated pathways after vancomycin treatment

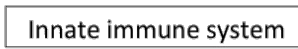

Adaptive immune system

\begin{tabular}{|l|l|}
\hline MHC-I & TK cell \\
\hline
\end{tabular}

Figure S2.6. Heatmap of 7 days placebo, amoxicillin and vancomycin-induced effects on abdominal subcutaneous adipose tissue gene expression. (A) Upregulated genes, (B) downregulated genes determined in adipose tissue biopsies $(n=30)$. The heatmap shows pathways related to metabolic function (Kyoto Encyclopedia of Genes and Genomes (KEGG) database) derived from Gene Set Enrichment Analysis. Signal log-ratio's (SLR) of genes in the heatmap represent genes that significantly contribute to the described pathways (FDR q-value <0.2). Color in the heatmap reflects the SLR per subject and group, with blue color being downregulated and red color being upregulated genes following treatment. (C) Schematic illustration of pathways that are upregulated and downregulated after vancomycin treatment as compared to placebo. 


\section{Table S2.1. Significantly different microbial taxa after intervention with vancomycin and placebo in feces using linear mixed models.}

\begin{tabular}{|c|c|c|c|c|}
\hline & $\mathrm{BH}_{-}$ & $\mathbf{P}$ & log fold change within & log fold change within \\
\hline Coprococcus.eutactus.et.rel & $1.46 \mathrm{E}-$ & $1.91 \mathrm{E}-$ & 162.262 .964 & -0.036098988 \\
\hline Ruminococcus.bromii.et.rel. & $4 \cdot 57 \mathrm{E}-$ & 1.09E- & 152.679 .587 & -0.338380905 \\
\hline Faecalibacterium.prausnitzii & $1.52 \mathrm{E}-$ & $2.35 \mathrm{E}-$ & 14.508 .797 & 0.075849958 \\
\hline Lactobacillus.plantarum.et. & $6.98 \mathrm{E}-$ & $5 \cdot 37 \mathrm{E}-$ & -139.338 .187 & -0.026993041 \\
\hline Eubacterium.hallii.et.rel. & $2.96 \mathrm{E}-$ & $1.25 \mathrm{E}-$ & 133.243 .286 & -0.114103865 \\
\hline Ruminococcus.obeum.et.rel & $1.20 \mathrm{E}-$ & $1.29 \mathrm{E}-$ & 125.172 .537 & -0.051553 \\
\hline Lachnospira.pectinoschiza. & $1.07 \mathrm{E}-$ & $1.07 \mathrm{E}-$ & 119.017 .372 & -0.144882415 \\
\hline Clostridium.cellulosi.et.rel. & $9.21 \mathrm{E}-$ & $8.50 \mathrm{E}-$ & 114.016 .546 & -0.267960043 \\
\hline Clostridium.nexile.et.rel. & $1.51 \mathrm{E}-$ & $2.21 \mathrm{E}-$ & 1.103 .251 & -0.158269081 \\
\hline Anaerostipes.caccae.et.rel. & $2.73 \mathrm{E}-$ & $4.83 \mathrm{E}-$ & 108.875 .593 & -0.225845465 \\
\hline Dorea.formicigenerans.et.re & $4.03 \mathrm{E}-$ & $9 \cdot 31 \mathrm{E}-$ & 105.973 .413 & -0.123118158 \\
\hline Veillonella & $9.45 \mathrm{E}-$ & $2.91 \mathrm{E}-$ & -0.99977749 & 0.008042942 \\
\hline Clostridium.sphenoides.et.r & $1.44 \mathrm{E}-$ & $1.77 \mathrm{E}-$ & 0.91030989 & -0.036075241 \\
\hline Serratia & $3 \cdot 30 \mathrm{E}-$ & 7.11E- & -0.85859596 & -0.132395735 \\
\hline Papillibacter.cinnamivorans & $3.21 \mathrm{E}-$ & $6.66 \mathrm{E}-$ & 0.82835965 & -0.156871014 \\
\hline Klebisiella.pneumoniae.et.r & $1.92 \mathrm{E}-$ & $3.15 \mathrm{E}-$ & -0.8215981 & -0.144113787 \\
\hline Megasphaera.elsdenii.et.rel & $7.84 \mathrm{E}-$ & $1.81 \mathrm{E}-$ & -0.8001288 & -0.016009582 \\
\hline Escherichia.coli.et.rel. & $6.70 \mathrm{E}-$ & $4.64 \mathrm{E}-$ & -0.79077723 & -0.086406762 \\
\hline Lachnobacillus.bovis.et.rel. & $3.21 \mathrm{E}-$ & $6.43 \mathrm{E}-$ & 0.78609501 & -0.130157333 \\
\hline Enterobacter.aerogenes.et.r & $3.21 \mathrm{E}-$ & $6.48 \mathrm{E}-$ & -0.76953625 & -0.145197198 \\
\hline Anaerovorax.odorimutans.e & $1.47 \mathrm{E}-$ & $2.04 \mathrm{E}-$ & 0.66699492 & -0.054689642 \\
\hline Sutterella.wadsworthia.et.r & $3.21 \mathrm{E}-$ & $6.35 \mathrm{E}-$ & -0.54038979 & -0.096457386 \\
\hline Granulicatella & $2.96 \mathrm{E}-$ & $1.37 \mathrm{E}-$ & -0.49661041 & -0.135840177 \\
\hline Lactobacillus.salivarius.et.r & 4.99E- & $1.23 \mathrm{E}-$ & -0.3206781 & 0.003000548 \\
\hline Collinsella & $7.84 \mathrm{E}-$ & $1.72 \mathrm{E}-$ & 0.30211314 & -0.045063251 \\
\hline Eubacterium.cylindroides.et & $3.56 \mathrm{E}-$ & $7.93 \mathrm{E}-$ & -0.28758184 & -0.020381399 \\
\hline Clostridium.difficile.et.rel. & $5.00 \mathrm{E}-$ & $3.07 \mathrm{E}-$ & -0.26460033 & -0.082852343 \\
\hline Bifidobacterium & $1.27 \mathrm{E}-$ & $1.47 \mathrm{E}-$ & 0.18320543 & -0.092371693 \\
\hline Eggerthella.lenta.et.rel. & $7.26 \mathrm{E}-$ & $6.14 \mathrm{E}-$ & 0.15800988 & -0.079697689 \\
\hline Aerococcus & $1.92 \mathrm{E}-$ & $3.25 \mathrm{E}-$ & -0.06422419 & -0.034350517 \\
\hline Atopobium & $3.79 \mathrm{E}-$ & $2.04 \mathrm{E}-$ & 0.02943863 & 0.005546336 \\
\hline
\end{tabular}


Table S2.2. The effect of 7 days vancomycin treatment confirmed by Random Forests analysis.

\begin{tabular}{|c|c|c|c|}
\hline Bacteria & MDA & Bacteria & MDA \\
\hline Klebsiella pneumoniae et rel. & $2,15 E-02$ & Bacteroides stercoris et rel. & $1,94 E-03$ \\
\hline Clostridium leptum et rel. & $1,33 E-02$ & Outgrouping Clostridium cluster XIVa & $1,89 E-03$ \\
\hline Clostridium nexile et rel. & $1,05 E-02$ & Streptococcus mitis et rel. & $1,68 E-03$ \\
\hline Lachnobacillus bovis et rel. & $9,86 E-03$ & Yersinia et rel. & $1,62 E-03$ \\
\hline Megasphaera elsdenii et rel. & $9,51 E-03$ & Bryantella formatexigens et rel. & $1,58 E-03$ \\
\hline Serratia & $9,40 E-03$ & Subdoligranulum variable at rel. & $1,49 E-03$ \\
\hline Veillonella & $9,20 E-03$ & Clostridium sphenoides et rel. & $1,48 E-03$ \\
\hline Eubacterium hallii et rel. & $8,77 E-03$ & Papillibacter cinnamivorans et rel. & $1,33 E-03$ \\
\hline Clostridium symbiosum et rel. & $8,58 E-03$ & Granulicatella & $1,21 E-03$ \\
\hline Prevotella tannerae et rel. & $8,40 E-03$ & Streptococcus intermedius et rel. & $1,15 E-03$ \\
\hline Uncultured Clostridiales I & $8,34 E-03$ & Eubacterium biforme et rel. & $1,13 E-03$ \\
\hline Lachnospira pectinoschiza et rel. & $7,44 E-03$ & Clostridium stercorarium et rel. & $1,11 E-03$ \\
\hline Ruminococcus gnavus et rel. & $7,22 E-03$ & Coprococcus eutactus et rel. & $8,78 E-04$ \\
\hline Ruminococcus lactaris et rel. & $6,77 E-03$ & Faecalibacterium prausnitzii et rel. & $6,84 E-04$ \\
\hline Enterobacter aerogenes et rel. & $5,39 E-03$ & Eubacterium rectale et rel. & $6,58 E-04$ \\
\hline Dorea formicigenerans et rel. & $5,34 E-03$ & Actinomycetaceae & $6,53 E-04$ \\
\hline Uncultured Clostridiales II & $4,61 E-03$ & Streptococcus bovis et rel. & $6,21 E-04$ \\
\hline Sporobacter termitidis et rel. & $4,45 E-03$ & Coprobacillus catenaformis et rel. & $6,10 E-04$ \\
\hline Lactobacillus plantarum et rel. & $4,37 E-03$ & Bacteroides splachnicus et rel. & $5,73 E-04$ \\
\hline Bacteroides vulgatus et rel. & $3,98 E-03$ & Uncultured Mollicutes & $5,58 E-04$ \\
\hline Eubacterium ventriosum et rel. & $3,74 E-03$ & Anaerofustis & $5,53 E-04$ \\
\hline Bacteroides plebeius et rel. & $3,64 E-03$ & Eubacterium cylindroides et rel. & $5,34 E-04$ \\
\hline Butyrivibrio crossotus et rel. & $3,46 E-03$ & Aneurinibacillus & $5,03 E-04$ \\
\hline Anaerostipes caccae et rel. & $3,41 E-03$ & Bacteroides ovatus et rel. & $4,95 E-04$ \\
\hline Anaerovorax odorimutans et rel. & $3,08 E-03$ & Methylobacterium & $3,98 E-04$ \\
\hline Clostridium orbiscindens et rel. & $3,02 E-03$ & Eubacterium siraeum et rel. & $3,58 E-04$ \\
\hline Escherichia coli et rel. & $3,01 E-03$ & Peptococcus niger et rel. & $2,72 E-04$ \\
\hline Anaerotruncus colihominis et rel. & $2,98 E-03$ & Collinsella & $2,48 E-04$ \\
\hline Clostridium cellulosi et rel. & $2,87 E-03$ & Prevotella melaninogenica et rel. & $2,38 E-04$ \\
\hline Bacteroides uniformis et rel. & $2,71 E-03$ & Eggerthella lenta et rel. & $2,37 E-04$ \\
\hline Ruminococcus obeum et rel. & $2,68 E-03$ & Catenibacterium mitsuokai et rel. & $2,27 E-04$ \\
\hline Ruminococcus bromii et rel. & $2,47 E-03$ & Burkholderia & $1,87 E-04$ \\
\hline Oscillospira guillermondii et rel. & $2,40 E-03$ & Vibrio & $1,74 E-04$ \\
\hline Roseburia intestinalis et rel. & $2,39 E-03$ & Uncultured Chroococcales & $1,38 E-04$ \\
\hline Bacteroides intestinalis et rel. & $2,12 E-03$ & Brachyspira & $1,35 E-04$ \\
\hline Ruminococcus callidus et rel. & $1,99 E-03$ & & \\
\hline
\end{tabular}

This tables shows the mean decrease accuracy of bacterial taxa that were also found to be altered after 7 days vancomycin treatment as determined by paired Wilcoxon test. MDA (mean decrease accuracy) represents the impact of bacterial taxa on the accuracy of the model. 
Table S2.3. Effects of vancomycin and amoxicillin vs. placebo on gene set enrichment of the adipose tissue

\begin{tabular}{|c|c|c|c|c|c|}
\hline \multirow{2}{*}{$\begin{array}{l}\text { CLASS } \\
\text { KEGG BRITE }\end{array}$} & \multirow{2}{*}{$\begin{array}{l}\text { UPREGULATED GENE SETS } \\
\text { KEGG PATHWAYS }\end{array}$} & \multicolumn{2}{|c|}{ VANCO-PLA } & \multicolumn{2}{|c|}{ AMOX-PLA } \\
\hline & & NES & FDR-q & NES & FDR-q \\
\hline $\begin{array}{l}\text { Energy } \\
\text { metabolism }\end{array}$ & OXIDATIVE.PHOSPHORYLATION & $\begin{array}{l}2.24 \\
*\end{array}$ & 0.000 & $1.75^{*}$ & 0.081 \\
\hline \multirow[t]{6}{*}{$\begin{array}{l}\text { Carbohydrate } \\
\text { metabolism }\end{array}$} & GLYCOLYSIS.GLUCONEOGENESIS & $\begin{array}{l}1.95 \\
*\end{array}$ & 0.006 & $1.81 *$ & 0.062 \\
\hline & GALACTOSE.METABOLISM & $\begin{array}{l}1.76 \\
*\end{array}$ & 0.023 & $2.02 *$ & 0.029 \\
\hline & PYRUVATE.METABOLISM & $\begin{array}{l}1.99 \\
*\end{array}$ & 0.006 & 1.38 & 0.263 \\
\hline & CITRATE.CYCLE.TCA.CYCLE. & $\begin{array}{l}1.83 \\
*\end{array}$ & 0.016 & $1.86 *$ & 0.049 \\
\hline & PROPANOATE.METABOLISM & $\begin{array}{l}1.89 \\
*\end{array}$ & 0.010 & 1.39 & 0.258 \\
\hline & STARCH.AND.SUCROSE.METABOLISM & 1.51 & 0.113 & $1.74^{*}$ & 0.079 \\
\hline \multirow{4}{*}{$\begin{array}{l}\text { Lipid } \\
\text { metabolism }\end{array}$} & ARACHIDONIC.ACID.METABOLISM & 0.97 & 0.719 & $1.74^{*}$ & 0.081 \\
\hline & FATTY.ACID.DEGRADATION & $\begin{array}{l}1.86 \\
*\end{array}$ & 0.014 & 1.14 & 0.522 \\
\hline & ETHER.LIPID.METABOLISM & $\begin{array}{l}1.84 \\
*\end{array}$ & 0.015 & 0.94 & 0.804 \\
\hline & PPAR.SIGNALING.PATHWAY & $\begin{array}{l}1.89 \\
*\end{array}$ & 0.010 & 0.85 & 0.889 \\
\hline \multirow[t]{7}{*}{$\begin{array}{l}\text { Amino acid } \\
\text { metabolism }\end{array}$} & LYSINE.DEGRADATION & $\begin{array}{l}1.87 \\
*\end{array}$ & 0.012 & 0.89 & 0.852 \\
\hline & CYSTEINE.AND.METHIONINE.METABOLISM & $\begin{array}{l}1.89 \\
*\end{array}$ & 0.010 & $1.76 *$ & 0.078 \\
\hline & TYROSINE.METABOLISM & $\begin{array}{l}1.83 \\
*\end{array}$ & 0.016 & $1.72 *$ & 0.083 \\
\hline & VALINE.LEUCINE.AND.ISOLEUCINE.DEGRADATION & $\begin{array}{l}1.79 \\
*\end{array}$ & 0.018 & 1.59 & 0.144 \\
\hline & GLYCINE.SERINE.AND.THREONINE.METABOLISM & $1.73^{*}$ & 0.030 & 1.6 & 0.137 \\
\hline & LYSOSOME & $\begin{array}{l}1.99 \\
*\end{array}$ & 0.006 & $2.22 *$ & 0.000 \\
\hline & COMPLEMENT.AND.COAGULATION.CASCADES & $\begin{array}{l}2.21 \\
*\end{array}$ & 0.001 & 1.6 & 0.133 \\
\hline \multirow[t]{2}{*}{$\begin{array}{l}\text { Digestive } \\
\text { system }\end{array}$} & PROTEIN.DIGESTION.AND.ABSORPTION & $\begin{array}{l}2.01 \\
*\end{array}$ & 0.006 & n.a. & n.a. \\
\hline & MINERAL.ABSORPTION & $\begin{array}{l}1.86 \\
*\end{array}$ & 0.013 & $1.79 *$ & 0.068 \\
\hline $\begin{array}{l}\text { Immune } \\
\text { system }\end{array}$ & INTESTINAL.IMMUNE.NETWORK.FOR.IGA.PRODUCTION & n.a. & n.a. & $2 *$ & 0.027 \\
\hline (MHC-II) & ANTIGEN.PROCESSING.AND.PRESENTATION & n.a. & n.a. & $1.8 *$ & 0.059 \\
\hline
\end{tabular}




\begin{tabular}{|c|c|c|c|c|c|}
\hline \multirow[t]{3}{*}{$\begin{array}{l}\text { Xenobiotics } \\
\text { metabolism }\end{array}$} & DRUG.METABOLISM.CYTOCHROME.P450 & $\begin{array}{l}2.09 \\
*\end{array}$ & 0.003 & 1.61 & 0.131 \\
\hline & METABOLISM.OF.XENOBIOTICS.BY.CYTOCHROME.P45O & $\begin{array}{l}1.98 \\
*\end{array}$ & 0.006 & 1.42 & 0.237 \\
\hline & DRUG.METABOLISM.OTHER.ENZYMES & 1.07 & 0.621 & $1.68 *$ & 0.100 \\
\hline $\begin{array}{l}\text { Cofactor and } \\
\text { vitamin } \\
\text { metabolism }\end{array}$ & RETINOL.METABOLISM & $\begin{array}{l}1.99 \\
*\end{array}$ & 0.006 & 1.12 & 0.560 \\
\hline $\begin{array}{l}\text { Signaling } \\
\text { molecules }\end{array}$ & ECM.RECEPTOR.INTERACTION & $1.77^{*}$ & 0.021 & 0.82 & 0.920 \\
\hline CLASS & DOWNREGULATED GENE SETS & VANCO & -PLA & AMOX- & PLA \\
\hline KEGG BRITE & KEGG PATHWAYS & NES & $\begin{array}{l}\text { FDR- } \\
\mathrm{q}\end{array}$ & NES & FDR-q \\
\hline \multirow{6}{*}{$\begin{array}{l}\text { Immune } \\
\text { system }\end{array}$} & NATURAL.KILLER.CELL.MEDIATED.CYTOTOXICITY & $-2.4^{*}$ & 0.001 & -0.74 & 1.000 \\
\hline & B.CELL.RECEPTOR.SIGNALING.PATHWAY & $-1.8 *$ & 0.026 & -0.76 & 1.000 \\
\hline & T.CELL.RECEPTOR.SIGNALING.PATHWAY & $-2.23^{*}$ & 0.002 & -0.77 & 1.000 \\
\hline & NOD.LIKE.RECEPTOR.SIGNALING.PATHWAY & - & 0.003 & -1 & 0.831 \\
\hline & & $2.24^{*}$ & & & \\
\hline & HEMATOPOIETIC.CELL.LINEAGE & $-1.72 *$ & 0.039 & n.a. & n.a. \\
\hline \multirow{5}{*}{$\begin{array}{l}\text { Immune } \\
\text { diseases } \\
\text { (MHC-I) }\end{array}$} & ALLOGRAFT.REJECTION & $-1.55^{*}$ & 0.078 & n.a. & n.a. \\
\hline & GRAFT.VERSUS.HOST.DISEASE & $-1.66 *$ & 0.053 & n.a. & n.a. \\
\hline & PRIMARY.IMMUNODEFICIENCY & $2.08 *$ & 0.006 & n.a. & n.a. \\
\hline & INFLAMMATORY.BOWEL.DISEASE.IBD. & $-1.55^{*}$ & 0.079 & n.a. & n.a. \\
\hline & SYST.L.ERYTHEM. (GENES OF HISTONE CLUSTER) & $-1.71 *$ & 0.040 & -1.35 & 0.489 \\
\hline $\begin{array}{l}\text { Substance } \\
\text { dependence }\end{array}$ & ALCOHOLISM (GENES OF HISTONE CLUSTER) & $-1.85^{*}$ & 0.023 & $-1.88 *$ & 0.085 \\
\hline $\begin{array}{l}\text { Replication and } \\
\text { repair }\end{array}$ & DNA.REPLICATION & $-1.57^{*}$ & 0.072 & n.a. & n.a. \\
\hline $\begin{array}{l}\text { Cell growth } \\
\text { and death }\end{array}$ & APOPTOSIS & $-1.99^{*}$ & 0.010 & n.a. & n.a. \\
\hline \multirow{2}{*}{$\begin{array}{l}\text { Signal } \\
\text { transduction }\end{array}$} & NF.KAPPA.B.SIGNALING.PATHWAY & $-1.56 *$ & 0.076 & n.a. & n.a. \\
\hline & TNF.SIGNALING.PATHWAY & $-1.63^{*}$ & 0.061 & n.a. & n.a. \\
\hline
\end{tabular}

Gene set enrichment analysis found enriched pathways of the KEGG (Kyoto Encyclopedia of Genes and Genomes) database. KEGG BRITE denotes clustering of KEGG-pathways in functional hierarchies, NES normalized enrichment score, FDR-q false discovery rate adjusted p-value, not appliccable means no significant upregulation, respectively downregulation after vancomycin or amoxicillin of the selected pathway. Data of $n=10$ per group was used for microarray analysis. * FDR-q<0.1, significant enrichment of the selected pathway. 


\section{References}

1

2

Khan, M. T., Nieuwdorp, M. \& Backhed, F. Microbial modulation of insulin sensitivity. Cell metabolism 20, 753-760, doi:10.1016/j.cmet.2014.07.006 (2014). Greenhill, C. Obesity: Gut microbiota, host genetics and diet interact to affect the risk of developing obesity and the metabolic syndrome. Nature reviews. Endocrinology, doi:10.1038/nrendo.2015.152 (2015).

Turnbaugh, P. J. et al. An obesity-associated gut microbiome with increased capacity for energy harvest. Nature 444, 1027-1031, doi:10.1038/nature05414 (2006).

Backhed, F. et al. The gut microbiota as an environmental factor that regulates fat storage. Proc Natl Acad Sci U S A 101, 15718-15723, doi:10.1073/pnas.0407076101 (2004).

Vrieze, A. et al. Fecal transplant: a safe and sustainable clinical therapy for restoring intestinal microbial balance in human disease? Best practice $\&$ research. Clinical gastroenterology 27, 127-137, doi:10.1016/j.bpg.2013.03.003 (2013).

Hartstra, A. V., Bouter, K. E., Backhed, F. \& Nieuwdorp, M. Insights into the role of the microbiome in obesity and type 2 diabetes. Diabetes care 38, 159-165, doi:10.2337/dc14-0769 (2015).

Cox, L. M. \& Blaser, M. J. Pathways in microbe-induced obesity. Cell metabolism 17, 883-894, doi:10.1016/j.cmet.2013.05.004 (2013).

Marchesi, J. R. et al. The gut microbiota and host health: a new clinical frontier. Gut, doi:10.1136/gutjnl-2015-309990 (2015).

Jones, M. L., Martoni, C. J., Ganopolsky, J. G., Labbe, A. \& Prakash, S. The human microbiome and bile acid metabolism: dysbiosis, dysmetabolism, disease and intervention. Expert opinion on biological therapy 14, 467-482, doi:10.1517/14712598.2014.880420 (2014).

Canfora, E. E., Jocken, J. W. \& Blaak, E. E. Short-chain fatty acids in control of body weight and insulin sensitivity. Nature reviews. Endocrinology 11, 577-591, doi:10.1038/nrendo.2015.128 (2015).

Jess, T. Microbiota, antibiotics, and obesity. The New England journal of medicine 371, 2526-2528, doi:10.1056/NEJMcibr1409799 (2014).

Cox, L. M. \& Blaser, M. J. Antibiotics in early life and obesity. Nature reviews. Endocrinology 11, 182-190, doi:10.1038/nrendo.2014.210 (2015).

3 Cani, P. D. et al. Changes in gut microbiota control metabolic endotoxemiainduced inflammation in high-fat diet-induced obesity and diabetes in mice. Diabetes 57, 1470-1481, doi:10.2337/dbo7-1403 (2008).

4 Chou, C. J., Membrez, M. \& Blancher, F. Gut decontamination with norfloxacin and ampicillin enhances insulin sensitivity in mice. Nestle Nutrition workshop 
series. Paediatric programme 62, 127-137; discussion 137-140, doi:10.1159/000146256 (2008).

15 Hwang, I. et al. Alteration of gut microbiota by vancomycin and bacitracin improves insulin resistance via glucagon-like peptide 1 in diet-induced obesity. FASEB journal : official publication of the Federation of American Societies for Experimental Biology 29, 2397-2411, doi:10.1096/fj.14-265983 (2015). Membrez, M. et al. Gut microbiota modulation with norfloxacin and ampicillin enhances glucose tolerance in mice. FASEB journal : official publication of the Federation of American Societies for Experimental Biology 22, 2416-2426, doi:10.1096/fj.07-102723 (2008).

17 Murphy, E. F. et al. Divergent metabolic outcomes arising from targeted manipulation of the gut microbiota in diet-induced obesity. Gut 62, 220-226, doi:10.1136/gutjnl-2011-300705 (2013).

18 Vrieze, A. et al. Impact of oral vancomycin on gut microbiota, bile acid metabolism, and insulin sensitivity. Journal of hepatology 60, 824-831, doi:10.1016/j.jhep.2013.11.034 (2014).

19 Rajilic-Stojanovic, M. et al. Development and application of the human intestinal tract chip, a phylogenetic microarray: analysis of universally conserved phylotypes in the abundant microbiota of young and elderly adults. Environmental microbiology 11, 1736-1751, doi:10.1111/j.14622920.2009.01900.x (2009).

20 Yap, I. K. et al. Metabonomic and microbiological analysis of the dynamic effect of vancomycin-induced gut microbiota modification in the mouse. Journal of proteome research 7, 3718-3728, doi:10.1021/pr700864x (2008).

21 Bech-Nielsen, G. V. et al. Manipulation of the gut microbiota in $\mathrm{C}_{57 \mathrm{BL} / 6}$ mice changes glucose tolerance without affecting weight development and gut mucosal immunity. Research in veterinary science 92, 501-508, doi:10.1016/j.rvsc.2011.04.005 (2012).

22 Carvalho, B. M. et al. Modulation of gut microbiota by antibiotics improves insulin signalling in high-fat fed mice. Diabetologia 55, 2823-2834, doi:10.1007/s00125-012-2648-4 (2012).

23 Rune, I. et al. Ampicillin-improved glucose tolerance in diet-induced obese

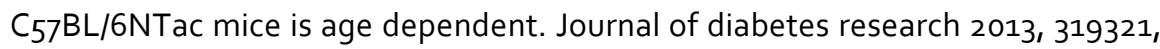
doi:10.1155/2013/319321 (2013).

24 Rajpal, D. K. et al. Selective Spectrum Antibiotic Modulation of the Gut Microbiome in Obesity and Diabetes Rodent Models. PloS one 10, e0145499, doi:10.1371/journal.pone.0145499 (2015). 
25 Mikkelsen, K. H. et al. Effect of Antibiotics on Gut Microbiota, Gut Hormones and Glucose Metabolism. PloS one 10, e0142352, doi:10.1371/journal.pone.0142352 (2015).

26 Thuny, F. et al. Vancomycin treatment of infective endocarditis is linked with recently acquired obesity. PLoS One 5, eg074, doi:10.1371/journal.pone.0009074 (2010).

27 Million, M. et al. Lactobacillus reuteri and Escherichia coli in the human gut microbiota may predict weight gain associated with vancomycin treatment. Nutrition \& diabetes 3, e87, doi:10.1038/nutd.2013.28 (2013).

28 Gonzales, M. et al. Faecal pharmacokinetics of orally administered vancomycin in patients with suspected Clostridium difficile infection. BMC infectious diseases 10, 363, doi:10.1186/1471-2334-10-363 (2010).

29 van Wijck, K. et al. Novel multi-sugar assay for site-specific gastrointestinal permeability analysis: a randomized controlled crossover trial. Clinical nutrition 32, 245-251, doi:10.1016/j.clnu.2012.06.014 (2013).

30 Cani, P. D. et al. Metabolic endotoxemia initiates obesity and insulin resistance. Diabetes 56, 1761-1772, doi:10.2337/dbo6-1491 (2007).

31 Ridlon, J. M., Kang, D. J. \& Hylemon, P. B. Bile salt biotransformations by human intestinal bacteria. Journal of lipid research 47, 241-259, doi:10.1194/jlr.R500013JLR200 (2006).

32 Brighton, C. A. et al. Bile acids trigger GLP-1 release predominantly by accessing basolaterally-located G-protein coupled bile acid receptors. Endocrinology, en20151321, doi:10.1210/en.2015-1321 (2015).

33 Gao, Z. et al. Butyrate improves insulin sensitivity and increases energy expenditure in mice. Diabetes 58, 1509-1517, doi:10.2337/dbo8-1637 (2009).

34 Ganal, S. C. et al. Priming of natural killer cells by nonmucosal mononuclear phagocytes requires instructive signals from commensal microbiota. Immunity 37, 171-186, doi:10.1016/j.immuni.2012.05.020 (2012).

35 Brestoff, J. R. \& Artis, D. Commensal bacteria at the interface of host metabolism and the immune system. Nature immunology 14, 676-684, doi:10.1038/ni.2640 (2013).

36 Fukuda, S. et al. Bifidobacteria can protect from enteropathogenic infection through production of acetate. Nature 469, 543-547, doi:10.1038/natureog646 (2011).

37 Furusawa, $\mathrm{Y}$. et al. Commensal microbe-derived butyrate induces the differentiation of colonic regulatory $T$ cells. Nature 504, 446-450, doi:10.1038/nature12721 (2013).

38 McCutcheon, A. L. Latent class analysis. (Sage, 1987). 
Mikkelsen, K. H., Knop, F. K., Frost, M., Hallas, J. \& Pottegard, A. Use of Antibiotics and Risk of Type 2 Diabetes: A Population-Based Case-Control Study. The Journal of clinical endocrinology and metabolism 100, 3633-3640, doi:10.1210/jc.2015-2696 (2015).

40 Kerr, C. A. et al. Early life events influence whole-of-life metabolic health via gut microflora and gut permeability. Critical reviews in microbiology 41, 326-340, doi:10.3109/1040841X.2013.837863 (2015).

41 Hulzebos, C. V. et al. Measurement of parameters of cholic acid kinetics in plasma using a microscale stable isotope dilution technique: application to rodents and humans. Journal of lipid research 42, 1923-1929 (2001).

42 Defronzo, R. A., Tobin, J. D. \& Andres, R. Glucose clamp technique: a method for quantifying insulin secretion and resistance. The American journal of physiology 237, E214-223 (1979).

43 Steele, R. Influences of glucose loading and of injected insulin on hepatic glucose output. Annals of the New York Academy of Sciences 82, 420-430 (1959).

44 Kotronen, A., Seppala-Lindroos, A., Bergholm, R. \& Yki-Jarvinen, H. Tissue specificity of insulin resistance in humans: fat in the liver rather than muscle is associated with features of the metabolic syndrome. Diabetologia $51,130-138$, doi:10.1007/s00125-007-0867-x (2008).

45 Brehm, A. et al. Increased lipid availability impairs insulin-stimulated ATP synthesis in human skeletal muscle. Diabetes 55, 136-140 (2006).

46 Most, J. et al. Combined epigallocatechin-3-gallate and resveratrol supplementation for $12 \mathrm{wk}$ increases mitochondrial capacity and fat oxidation, but not insulin sensitivity, in obese humans: a randomized controlled trial. The American journal of clinical nutrition, doi:10.3945/ajcn.115.122937 (2016).

47 Schoffelen, P. F., Westerterp, K. R., Saris, W. H. \& Ten Hoor, F. A dualrespiration chamber system with automated calibration. Journal of applied physiology 83, 2064-2072 (1997).

48 Weir, J. B. New methods for calculating metabolic rate with special reference to protein metabolism. The Journal of physiology 109, 1-9 (1949).

49 Frayn, K. N. Calculation of substrate oxidation rates in vivo from gaseous exchange. Journal of applied physiology: respiratory, environmental and exercise physiology 55, 628-634 (1983).

50 Jans, A. et al. PUFAs acutely affect triacylglycerol-derived skeletal muscle fatty acid uptake and increase postprandial insulin sensitivity. The American journal of clinical nutrition 95, 825-836, doi:10.3945/ajcn.111.028787 (2012).

51 Kersten, $\mathrm{S}$. et al. Caloric restriction and exercise increase plasma ANGPTL4 levels in humans via elevated free fatty acids. Arteriosclerosis, thrombosis, and vascular biology 29, 969-974, doi:10.1161/ATVBAHA.108.182147 (2009). 
Schols, A. M., Buurman, W. A., Staal van den Brekel, A. J., Dentener, M. A. \& Wouters, E. F. Evidence for a relation between metabolic derangements and increased levels of inflammatory mediators in a subgroup of patients with chronic obstructive pulmonary disease. Thorax 51, 819-824 (1996).

53 Vilsboll, T. et al. Incretin secretion in relation to meal size and body weight in healthy subjects and people with type 1 and type 2 diabetes mellitus. The Journal of clinical endocrinology and metabolism 88, 2706-2713, doi:10.1210/jc.2002-021873 (2003).

54 van Eijk, H. M., Bloemen, J. G. \& Dejong, C. H. Application of liquid chromatography-mass spectrometry to measure short chain fatty acids in blood. Journal of chromatography. B, Analytical technologies in the biomedical and life sciences 877, 719-724, doi:10.1016/j.jchromb.2009.01.039 (2009).

55 Goossens, G. H. et al. Increased adipose tissue oxygen tension in obese compared with lean men is accompanied by insulin resistance, impaired adipose tissue capillarization, and inflammation. Circulation 124, 67-76, doi:10.1161/CIRCULATIONAHA.111.027813 (2011).

56 Lin, K. et al. MADMAX - Management and analysis database for multiple omics experiments. Journal of integrative bioinformatics 8, 160, doi:10.239o/biecoll-jib2011-160 (2011).

57 Smyth, G. K. Linear models and empirical bayes methods for assessing differential expression in microarray experiments. Statistical applications in genetics and molecular biology 3, Article3, doi:10.2202/1544-6115.1027 (2004).

58 Garcia-Villalba, R. et al. Alternative method for gas chromatography-mass spectrometry analysis of short-chain fatty acids in faecal samples. Journal of separation science 35, 1906-1913, doi:10.1002/jssc. 201101121 (2012).

59 Lahti, L., Torrente, A., Elo, L. L., Brazma, A. \& Rung, J. A fully scalable online pre-processing algorithm for short oligonucleotide microarray atlases. Nucleic acids research 41, e110, doi:10.1093/nar/gkt229 (2013).

60 Lahti, L., Elo, L. L., Aittokallio, T. \& Kaski, S. Probabilistic analysis of probe reliability in differential gene expression studies with short oligonucleotide arrays. IEEE/ACM transactions on computational biology and bioinformatics / IEEE, ACM 8, 217-225, doi:10.1109/TCBB.2009.38 (2011).

61 Bates, D., Mächler, M., Bolker, B. \& Walker, S. Fitting Linear Mixed-Effects Models Using Ime4. Journal of Statistical Software 67, doi:doi:10.18637/jss.v067.io1 (2015).

62 Liaw, A. \& Wiener, M. Classification and Regression by randomForest. R News 2, 18-22 (2002).

63 Proust-Lima, C., Philipps, V. \& Liquet, B. Estimation of Extended Mixed Models Using Latent Classes and Latent Processes: the R package Icmm. (2015). 
64 Oksanen JFGB, K. R., Legendre P, Minchin PR, O'Hara RB, Simpson GL et al. . Package 'vegan' version 2.0-2. (2011). 


\section{Antibiotic treatment does not affect skeletal muscle substrate metabolism in obese men: a randomized, double-blind, placebo-controlled trial}

Dorien Reijnders

Gijs H Goossens

Ellen E Blaak

To be submitted 


\section{Abstract}

Aim To investigate the impact of gut microbiota manipulation on fasting and postprandial skeletal muscle metabolism in obese humans.

Methods 40 obese, insulin resistant males were randomized to amoxicillin (broad-spectrum antibiotic), vancomycin (targeting gram-positive bacteria) or placebo treatment ( $7 \mathrm{~d}, 1500 \mathrm{mg} / \mathrm{d})$. Before and after treatment, forearm blood flow and metabolite fluxes across forearm muscle were measured.

Results Neither vancomycin nor amoxicillin treatment significantly affected fasting and postprandial plasma glucose, free fatty acids (FFA), triacylglycerol (TAG), glycerol, lactate and insulin concentrations, and forearm blood flow. Fasting and postprandial net forearm muscle glucose uptake and the release of lactate across forearm were not significantly altered by antibiotic treatment as compared to placebo. Finally, antibiotic treatment did not change fasting and postprandial glycerol, FFA and TAG fluxes across forearm muscle.

Conclusion The present study demonstrated that skeletal muscle substrate metabolism, forearm blood flow and postprandial glucose metabolism were not affected following short-term antibiotic treatment in obese men with impaired glucose metabolism. 


\section{Introduction}

Accumulating evidence indicates that the composition of the gut microbiome is involved in host energy and substrate metabolism, thereby contributing to the etiology of obesity and type 2 diabetes mellitus $\left(T_{2} D M\right)^{1}$. The intestinal microbiota is involved in the secretion of various gut hormones and substrates, which in turn influence metabolic processes in peripheral organs such as the liver, adipose tissue and skeletal muscle. The skeletal muscle accounts for approximately $80 \%$ of insulin-stimulated glucose disposal and, therefore, plays a key role in development and progression of insulin resistance and $\mathrm{T}_{2} \mathrm{DM}^{2}$. In the obese state, a reduced adipose tissue lipid buffering capacity contributes to an increased supply of lipids to non-adipose tissues such as the skeletal muscle, causing ectopic lipid accumulation when oxidative capacity is insufficient ${ }^{3}$. In addition, impairments in postprandial fatty acid uptake, storage and oxidation may contribute to intramuscular accumulation of lipid-intermediates like diacylglycerol and ceramides ${ }^{4,5}$. Accumulation of these bioactive metabolites has been associated with insulin resistance.

Interestingly, the gut microbiota may affect host metabolic health through effects on skeletal muscle metabolism, at least in rodents. It has been shown that the persistent lean phenotype of germ-free mice may be associated with increased skeletal muscle levels of phosphorylated AMPK and its downstream targets involved in fatty acid oxidation ${ }^{6}$. Additionally, the gut hormones glucagon-like peptide 1 (GLP-1) and Peptide YY have been reported to affect muscle metabolism and insulin sensitivity in rats through effects on microvascular blood flow and fat oxidation, respectively?.

Finally, SCFA, derived from microbial fermentation of indigestible carbohydrates, may have effects on peripheral substrate metabolism since their receptors are expressed in skeletal muscle ${ }^{8,9}$. Taken together, several lines of evidence suggest that the gut microbiota-skeletal muscle cross-talk is involved in substrate metabolism and insulin sensitivity in rodents ${ }^{1,10}$. However, human studies that have investigated the effects of microbiota manipulation on skeletal muscle substrate metabolism and insulin sensitivity under physiological conditions are lacking. To unravel the impact of gut microbiota manipulation on fasting and postprandial skeletal muscle metabolism in humans, we investigated the effect of broad (amoxicillin; AMOX) and narrow-sprectrum (vancomycin; VANCO) 
antibiotic treatment on skeletal muscle blood flow and metabolite fluxes across forearm muscle in obese men with impaired glucose metabolism.

\section{Methods}

This study was performed in a sub-cohort of a larger clinical trial in which we investigated the impact of antibiotic treatment on microbiota composition and tissue-specific insulin sensitivity. In this randomized, placebo-controlled, doubleblind study with a 3 -armed parallel design, participants received either AMOX, VANCO $(1500 \mathrm{mg} / \mathrm{d})$ or placebo (PLA) for 7 consecutive days. The inclusion criteria are presented in detail before ${ }^{11}$. Here, the effects of gut microbiota manipulation on skeletal muscle substrate metabolism and postprandial insulin sensitivity were investigated in 40 obese men with impaired impaired fasting glucose and/or impaired glucose tolerance, who all gave written informed consent (reviewed and approved by the local Medical Ethical Committee). All procedures were according to the declaration of Helsinki (revised version, 2008)

\section{Study procedures}

Before and after the intervention, forearm muscle metabolism and blood flow were studied in the fasting state and for 4 hours after consumption of a high-fat mixed meal (HFMM; $2.6 \mathrm{MJ}$, [61 E\% fat, $33 \mathrm{E} \%$ carbohydrate, $6 \mathrm{E} \%$ protein] $)^{11}$. The evening before an investigation day, participants were asked to consume the same evening meal (low in fiber and fat) ${ }^{12}$. Skeletal muscle metabolism was studied in the forearm muscle, using arterio-venous concentration differences corrected for forearm blood flow. Therefore, catheters were inserted into the superficial dorsal hand vein, which was heated in a hot box $\left(55^{\circ} \mathrm{C}\right)$ to allow arterialized venous blood sampling, and retrogradely in a deep anticubital vein of the contralateral forearm to sample venous blood ${ }^{13}$. Blood samples were taken simultaneously from these catheters during fasting and postprandially ( $\mathrm{t} 3 \mathrm{O}, \mathrm{t} 6 \mathrm{O}$, t9o, t120, t18o, t240 min). During this timeframe, total forearm blood flow (FBF) was measured before each blood sampling by venous occlusion plethysmography with a mercury strain gauge (Periflow 0699; Janssen Scientific Instruments), as described previously ${ }^{14}$. To prevent contamination of the blood from the forearm vein with blood from the hand, a wrist cuff was inflated to 200 $\mathrm{mm} \mathrm{Hg}$ for $3 \mathrm{~min}$ before the samples were taken ${ }^{15}$. 


\section{Biochemical analyses}

Blood was collected in pre-chilled EDTA-tubes and immediately centrifuged $\left(1,000 \mathrm{~g}, 10 \mathrm{~min}, 4^{\circ} \mathrm{C}\right)$. Plasma was frozen in liquid nitrogen and stored at $-80^{\circ} \mathrm{C}$ until analysis. Plasma free fatty acids (FFA) were analyzed using standard enzymatic techniques automated on a Cobas Fara centrifugal spectrophotometer (Roche Diagnostics, Basel, Switzerland). Plasma triacylglycerol (TAG), glycerol, glucose, and lactate were analyzed enzymatically on a Cobas Mira automated spectrophotometer. Plasma insulin was measured with a radioimmunoassay (Millipore). Hematocrit was determined in heparinized blood using a microcapillary system.

\section{Calculations and statistics}

The power calculation for the total inclusion can be found elsewhere ${ }^{11}$. To be able to detect a $5 \%$ difference in skeletal muscle lipid handling, $n=12$ per intervention group were needed $^{16}$. To take into account block-randomization ( $n=6$ per block) and possible drop-outs, we included 40 participants for this sub-study.

The postprandial response is given as incremental area under the curve per minute, which was calculated by the trapezoid rule from the start of the meal to $240 \mathrm{~min}\left(\mathrm{iAUC}_{0-240}\right)$.

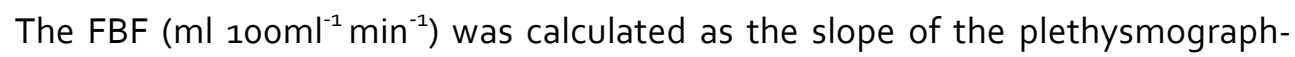
graph muliplied by 6 . The blood flow rate is FBF*(100-hematocrit/100). The forearm muscle fluxes of metabolites (i.e. glucose uptake, lactate release) were calculated by the arteriovenous difference of glucose multiplied by the blood flow rate. A positive flux indicates net uptake across forearm muscle whereas a negative flux indicates net release into the circulation.

Analysis of variance (ANOVA) was applied to compare group characteristics at baseline. Differences between treatments were analyzed using repeatedmeasures ANOVA with time and treatment as factors. Data are expressed as mean \pm SEM. $P<0.05$ (two-sided P-value) was considered to be statistically significant. Statistical analysis was performed using SPSS for Mac version 20.0 (Chicago, IL, USA). 


\section{Results}

No serious adverse events of the antibiotic treatment were reported. At baseline, body weight, BMI, and body fat distribution did not differ between groups (Table 3.1). Furthermore, baseline fasting plasma glucose, insulin, lactate, glycerol, TAG and FFA concentrations were comparable between groups (Figure 3.1). As expected, plasma glucose, lactate, insulin and TAG concentrations increased compared to baseline levels after meal intake in all groups (Figure 3.1). Furthermore, meal intake decreased postprandial FFA and free glycerol concentrations, which returned to baseline values after 240 minutes.

VANCO and AMOX treatment did not significantly affect fasting arterialized concentrations and the postprandial response (iAUC/min) of circulating metabolites and insulin as compared to PLA (all P > 0.05).

Baseline fasting FBF was comparable between groups (mean FBF, $4.48 \pm 0.23 \mathrm{ml}$ $100 \mathrm{ml}^{-1} \mathrm{~min}^{-1}$ ), and postprandial FBF did not significantly increase after meal intake. Antibiotic treatment had no significant effect on baseline and postprandial FBF as compared to PLA (Figure 3.1).

We found neither significant effects of VANCO or AMOX on fasting and postprandial net forearm muscle glucose uptake nor the release of lactate across forearm muscle as compared to PLA. Similarly, antibiotic treatment did not alter fasting and postprandial glycerol, FFA and TAG fluxes across forearm muscle (Figure 3.1).

Table 3.1. Subject characteristics

\begin{tabular}{llll}
\hline & PLA $(\mathrm{n}=13)$ & AMOX $(\mathrm{n}=12)$ & VANCO $(\mathrm{n}=13)$ \\
\hline Age (years) & $62.6 \pm 1.3$ & $56.3 \pm 2.0$ & $60.0 \pm 1.9$ \\
Body Weight $(\mathrm{kg})$ & $92.0 \pm 2.4$ & $96.7 \pm 2.9$ & $96.5 \pm 2.5$ \\
BMI $(\mathrm{kg} / \mathrm{m} 2)$ & $30.0 \pm 0.6$ & $31.3 \pm 1.1$ & $31.2 \pm 0.8$ \\
WHR & $1.05 \pm 0.01$ & $1.04 \pm 0.02$ & $1.07 \pm 0.02$ \\
Waist circumference $(\mathrm{cm})$ & $108.3 \pm 2.1$ & $107.7 \pm 3.4$ & $111.5 \pm 3.0$ \\
Fasting glucose $(\mathrm{mmol} / \mathrm{l})$ & $6.3 \pm 0.1$ & $6.5 \pm 0.2$ & $6.3 \pm 0.2$ \\
Fasting insulin $(\mathrm{mU} / \mathrm{l})$ & $11.5 \pm 1.3$ & $12.6 \pm 1.3$ & $14.3 \pm 1.8$ \\
HOMA-IR & $3.3 \pm 0.3$ & $3.1 \pm 0.4$ & $3.9 \pm 0.3$ \\
\hline
\end{tabular}

Data are expressed as mean \pm SEM. No significant differences were found between groups (one-way ANOVA, $P>0.05$ ) 

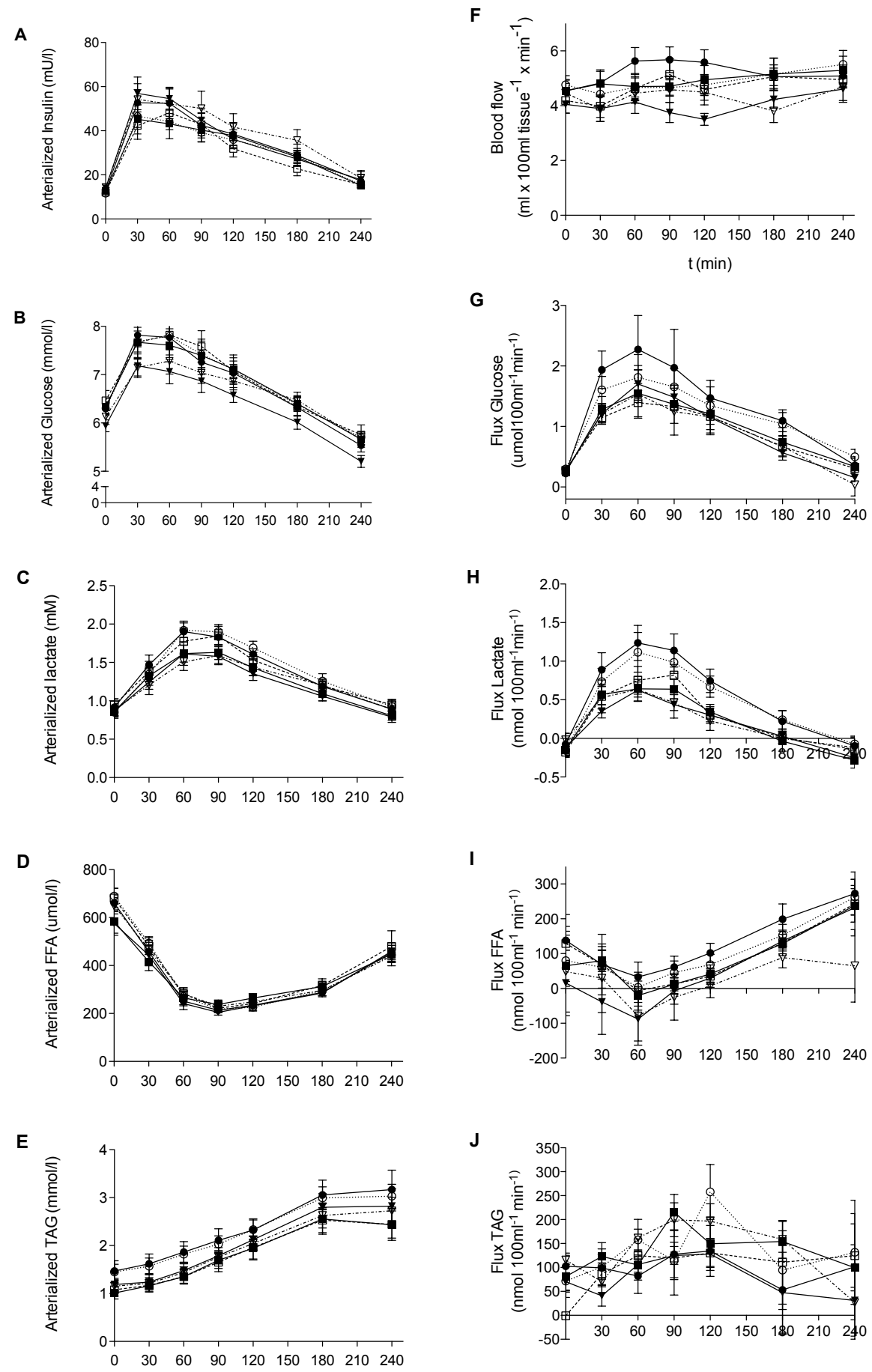
Figure 3.1. Effect of 7 days PLA, AMOX or VANCO treatment on plasma concentrations and fluxes of glucose, lactate, FFA, TAG and insulin across forearm muscle. Fasting $(\mathrm{t}=\mathrm{O})$ and postprandial concentrations of $(\mathrm{A})$ arterialized insulin, (B) glucose, (C) lactate, (D) free fatty acids (FFA) and (E) triacylglycerol (TAG) concentrations were not affected by AMOX or VANCO as assessed by repeated measures ANOVA. AMOX and VANCO did not affect forearm blood flow (F) and net fluxes of (G) glucose, (H) lactate, (I) FFA, and (J) TAG across forearm muscle are shown. A positive flux indicates net uptake across the forearm muscle whereas a negative flux indicates net release. Values are given as mean $\pm \operatorname{SEM}(n=37)$.

\section{Discussion}

The present study demonstrated that gut microbiota manipulation using antibiotics had no significant effects on fasting and postprandial forearm muscle substrate metabolism and postprandial forearm muscle insulin sensitivity in obese men with impaired glucose metabolism.

VANCO reduced microbiota diversity after 7 days treatment, as we have previously reported ${ }^{11}$. The abundance of butyrate-producing species and species involved in bile acid dehydroxylation decreased following VANCO, whilst there was a compensatory upregulation of gram-negative Proteobacteria and VANCOresistant bacilli. AMOX did not induce any significant changes in microbiota diversity or composition, and did not affect related metabolites ${ }^{11}$.

Here, we found that antibiotic treatment did neither influence systemic lipid overflow, i.e. fasting and postprandial TAG and FFA concentrations, nor postprandial glucose and insulin concentrations. Together with an unchanged postprandial forearm muscle glucose flux following VANCO or AMOX, this indicates that postprandial insulin sensitivity did not change by antibiotic treatment. In contrast, rodents studies have indicated that the gut microbiota may exert important effects on substrate and energy metabolism, thereby contributing to the etiology of obesity and type 2 diabetes through various mechanisms. Alterations in gut hormones like GLP-1 and microbial products such as SCFA ${ }^{17,18}$ have been suggested to underlie changes in skeletal muscle fat oxidation and substrate metabolism following gut microbiota manipulation. More specific, it has been suggested that the stimulation of fat oxidation in skeletal muscle might be under influence of gut-derived hormones and substrates through metabolic pathways involving the phosporylation of AMPK ${ }^{6}$. 
Also, AMPK is able to regulate the transcription of the glucose transporter $\mathrm{GLUT}_{4}{ }^{19}$, thereby influencing skeletal muscle glucose uptake. However, it is important to emphasize that most data and putative mechanisms are obtained from animal studies.

Previously, we have reported that this 7-day VANCO treatment significantly reduced fecal SCFA (butyrate and acetate) and, to a lesser extent, plasma butyrate concentrations. Based on these findings, we hypothesized that this may induce alterations in skeletal muscle substrate metabolism. However, the present data clearly show that these changes in SCFA and BA concentrations neither affect skeletal muscle substrate fluxes nor postprandial insulin sensitivity. Another group of gut derived-signals, the incretins peptide $Y Y$ and GLP-1, might affect microvascular blood flow ${ }^{17,20}$, thereby altering substrate supply and uptake in skeletal muscle. In line with previously reported unchanged GLP-1 concentrations following antibiotic treatment ${ }^{11}$, the present data indicate that short-term antibiotic treatment did not alter fasting and postprandial forearm muscle blood flow.

\section{Conclusion}

The present study demonstrated that, despite marked changes in gut microbiota composition following VANCO treatment, skeletal muscle substrate metabolism and postprandial glucose metabolism were not altered by 7 -days antibiotic treatment in obese men with impaired glucose metabolism. Further research should establish whether more prolonged manipulation of the gut microbiota may induce changes in skeletal muscle substrate metabolism. 


\section{References}

1

2

Khan, M. T., Nieuwdorp, M. \& Backhed, F. Microbial modulation of insulin sensitivity. Cell metabolism 20, 753-760, doi:10.1016/j.cmet.2014.07.006 (2014). Thiebaud, D. et al. The effect of graded doses of insulin on total glucose uptake, glucose oxidation, and glucose storage in man. Diabetes 31, 957-963 (1982).

Stinkens, R., Goossens, G. H., Jocken, J. W. \& Blaak, E. E. Targeting fatty acid metabolism to improve glucose metabolism. Obesity reviews : an official journal of the International Association for the Study of Obesity 16, 715-757, doi:10.1111/obr.12298 (2015).

Farese, R. V., Jr., Yost, T. J. \& Eckel, R. H. Tissue-specific regulation of lipoprotein lipase activity by insulin/glucose in normal-weight humans. Metabolism: clinical and experimental 40, 214-216 (1991).

Ritter, O., Jelenik, T. \& Roden, M. Lipid-mediated muscle insulin resistance: different fat, different pathways? Journal of molecular medicine 93, 831-843, doi:10.1007/s00109-015-1310-2 (2015).

Backhed, F., Manchester, J. K., Semenkovich, C. F. \& Gordon, J. I. Mechanisms underlying the resistance to diet-induced obesity in germ-free mice. Proceedings of the National Academy of Sciences of the United States of America 104, 979-984, doi:10.1073/pnas.0605374104 (2007).

7 Canfora, E. E., Jocken, J. W. \& Blaak, E. E. Short-chain fatty acids in control of body weight and insulin sensitivity. Nature reviews. Endocrinology 11, 577-591, doi:10.1038/nrendo.2015.128 (2015).

Cornall, L. M., Mathai, M. L., Hryciw, D. H. \& McAinch, A. J. Diet-induced obesity up-regulates the abundance of GPR43 and GPR120 in a tissue specific manner. Cellular physiology and biochemistry : international journal of experimental cellular physiology, biochemistry, and pharmacology 28, 949-958, doi:10.1159/000335820 (2011).

9 Bonini, J. A., Anderson, S. M. \& Steiner, D. F. Molecular cloning and tissue expression of a novel orphan $G$ protein-coupled receptor from rat lung. Biochemical and biophysical research communications 234, 190-193, doi:10.1006/bbrc.1997.6591 (1997).

Cox, L. M. \& Blaser, M. J. Pathways in microbe-induced obesity. Cell metabolism 17, 883-894, doi:10.1016/j.cmet.2013.05.004 (2013).

Reijnders, D. et al. Effects of Gut Microbiota Manipulation by Antibiotics on Host Metabolism in Obese Humans: A Randomized Double-Blind Placebo-Controlled Trial. Cell metabolism 24, 63-74, doi:10.1016/j.cmet.2016.06.016 (2016).

Robertson, M. D., Henderson, R. A., Vist, G. E. \& Rumsey, R. D. Extended effects of evening meal carbohydrate-to-fat ratio on fasting and postprandial substrate metabolism. The American journal of clinical nutrition 75, 505-510 (2002). 
13 van Hees, A. M. et al. Skeletal muscle fatty acid handling in insulin resistant men. Obesity 19, 1350-1359, doi:10.1038/oby.2011.10 (2011).

14 Blaak, E. E. et al. Total forearm blood flow as an indicator of skeletal muscle blood flow: effect of subcutaneous adipose tissue blood flow. Clinical science 87, 559-566 (1994).

15 Robertson, M. D., Bickerton, A. S., Dennis, A. L., Vidal, H. \& Frayn, K. N. Insulinsensitizing effects of dietary resistant starch and effects on skeletal muscle and adipose tissue metabolism. The American journal of clinical nutrition 82, 559-567 (2005).

16 Moors, C. C., van der Zijl, N. J., Diamant, M., Blaak, E. E. \& Goossens, G. H. Impaired insulin sensitivity is accompanied by disturbances in skeletal muscle fatty acid handling in subjects with impaired glucose metabolism. International journal of obesity 36, 709-717, doi:10.1038/ijo.2011.123 (2012).

17 Chai, W. et al. Glucagon-like peptide 1 recruits microvasculature and increases glucose use in muscle via a nitric oxide-dependent mechanism. Diabetes 61, 888896, doi:10.2337/db11-1073 (2012).

18 Cherbut, $C$. et al. Short-chain fatty acids modify colonic motility through nerves and polypeptide $Y Y$ release in the rat. The American journal of physiology 275, G1415-1422 (1998).

19 McGee, S. L. \& Hargreaves, M. AMPK-mediated regulation of transcription in skeletal muscle. Clinical science 118, 507-518, doi:10.1042/CS20090533 (2010).

20 Samuel, B. S. et al. Effects of the gut microbiota on host adiposity are modulated by the short-chain fatty-acid binding $G$ protein-coupled receptor, Gpr41. Proceedings of the National Academy of Sciences of the United States of America 105, 16767-16772, doi:10.1073/pnas.0808567105 (2008). 


\section{Gut microbiota manipulation and lipolysis in human adipose tissue}

Dorien Reijnders Johan W.E. Jocken Emanuel E. Canfora Jogchum Plat Gijs H. Goossens Ellen E. Blaak 


\section{Abstract}

Background Recent evidence indicates that the intestinal microbiota and its products may contribute to the development of obesity and related cardiometabolic complications by affecting lipid metabolism and insulin sensitivity. Here, we investigated the effects of microbiota manipulation on ex vivo basal and $\beta$-adrenergically-stimulated lipolysis in human adipocytes, and adipose tissue gene and protein expression.

Methods Thirty-six obese men were randomly assigned to amoxicillin (AMOX; broad-spectrum antibiotic $n=12$ ), vancomycin (VANCO; targeting gram-positive bacteria $n=9)$ or placebo $(n=15)$ treatment $(7 d, 500 \mathrm{mg}$ t.i.d). Before and after treatment, ex vivo adipose tissue lipolysis was assessed under basal conditions and during stimulation with the non-selective $\beta$-agonist isoprenaline (ISO) using freshly isolated mature adipocytes. Glycerol release into the medium was measured as an indicator of lipolysis. In addition, gene (targeted microarray) and protein expression analyses were performed to provide insight into underlying pathways. Results Antibiotic treatment had no significant effects on basal and maximal ISO-mediated glycerol release from adipocytes. However, $\beta$-adrenergic sensitivity was slightly improved after VANCO (EC50: $2.4 \times 10^{-6} \pm 5.7 \times 10^{-7} \mathrm{M}$ vs $\left.1.0 \times 10^{-6} \pm 5.9 \times 10^{-7} \mathrm{M}, \quad P<0.05\right)$. Surprisingly, this was not accompanied by alterations in $\beta$-adrenoceptor expression or post-receptor signaling (i.e. hormone-sensitive lipase-phosphorylation).

Conclusion We demonstrate that short-term antibiotic treatment by VANCO slightly increased adipose tissue $\beta$-adrenergic sensitivity. Whether this translates in an improved adipose tissue function in the long term remains to be determined. 


\section{Introduction}

The intestinal microbiota seems to contribute to the development of obesity and related cardiometabolic complications, at least in rodents ${ }^{1-3}$. Gut microbial products and their stimulation of gut hormonal secretion may influence metabolic processes in the skeletal muscle, liver and adipose tissue, thereby affecting lipid storage $\mathrm{e}^{4,5}$ and lipolysis ${ }^{6}$. The release of lipids from adipose tissue of obese insulin resistant individuals is characterized by a blunted catecholamineinduced lipolysis, which is related to reduced $\beta$-adrenoceptor expression and decreased lipase activation ${ }^{7,8}$. This impaired lipolytic response might contribute to adipose tissue dysfunction and, as a consequence, the development of insulin resistance ${ }^{9}$.

The gut microbiota might affect adipose tissue lipid turnover through multiple mechanisms, including altered production of angiopoietin-like protein 4 (ANGPTL4), short-chain fatty acids (SCFA), bile acids (BA), lipopolysaccharides (LPS), as well as gut-derived incretins ${ }^{3}$. First, the gut microbiota regulates the production of ANGPTL4, also known as Fasting-Induced Adipose Factor, which is a circulating antagonist of lipoprotein lipase (LPL) that is present in adipose tissue, liver and the intestinal tract. Additionally, ANGPTL4 promotes the intracellular lipolytic response to fasting and catecholamines in murine adipocytes ${ }^{10}$. In line, human in vivo studies have shown that plasma ANGPTL4 concentrations were positively associated with fasting levels of free fatty acids (FFA) and adipose tissue lipolysis ${ }^{11}$. Another potential mechanism through which our gut can affect host lipid metabolism is fermentation of nondigestable carbohydrates to SCFA in the distal ileum, cecum and colon. These SCFA, of which acetate, butyrate and propionate are the most abundant, are ligands for the G-protein-coupled receptors (GPCRs), GPCR 41 and GPCR 43, which are expressed on several peripheral tissues including adipose tissue ${ }^{12}$. Several human studies have shown that oral or rectal administration of SCFA, in particular acetate, significantly decreased plasma FFA concentrations ${ }^{13-15}$. In addition, treatment of differentiated murine $3^{T} 3^{-}$L1 adipocytes with SCFA reduced fasting and catecholamine-mediated lipolysis via activation of $\mathrm{GPR}_{43} 3^{16}$. A decreased hormone-sensitive lipase (HSL) phosphorylation may underlie this antilipolytic effect of SCFA, as has recently been shown in murine $3^{T} 3^{-L 1}$ adipocytes ${ }^{17}$. Finally, also gut-derived $B A^{18,19}$ and incretins, including glucagon-like peptide $1(G L P-1)^{20}$, might affect adipose tissue lipid metabolism. Taken together, modulation of the 
gut microbiota and its products may provide a promising strategy to target adipose tissue lipolysis, thereby improving obesity-related metabolic impairments. Importantly, however, most data are based on in vivo and in vitro murine studies, whilst well-controlled human studies investigating the effect of microbiota modulation on adipose tissue lipolysis are currently lacking. Here, we investigated the effects of microbiota manipulation, achieved by oral broad or small-spectrum antibiotic treatment, in obese men with impaired glucose homeostasis on ex vivo basal and $\beta$-adrenergically stimulated lipolysis in human adipocytes. In addition, targeted gene and protein expression analyses in adipose tissue biopsies were performed to investigate potential underlying mechanisms.

\section{Methods}

\section{Study participants}

The current research was conducted as part of larger clinical trial on the effect of gut microbiota manipulation on host metabolism ${ }^{21}$. In addition, we included data from 12 subjects from a recently conducted study using the same antibiotics-

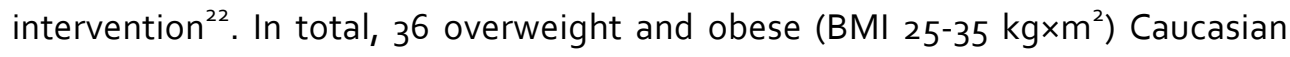
men between 35-70 years were included in the present study. Detailed inclusion and exclusion criteria have previously been described ${ }^{21,22}$. All subjects gave written informed consents for participation in both studies, which were reviewed and approved by the local Medical Ethical Committee of Maastricht University Medical Centre+. All procedures were according to the declaration of Helsinki (revised version, October 2008, Seoul, South Korea).

\section{Study design}

Study participants were randomized (double-blind) to the oral intake of amoxicillin (AMOX, broad-spectrum antibiotic), vancomycin (VANCO, directed against gram-positive bacteria) or placebo (PLA) for 7 consecutive days (500 mg t.i.d.). Participants were asked to maintain their habitual physical activity pattern and dietary habits throughout the study. An abdominal subcutaneous adipose tissue biopsy was taken under local anaesthesia under fasted conditions before and after 7 days antibiotics treatment for ex vivo characterization of adipocyte lipolysis, targeted microarray and Western Blot analyses, as described in more detail below. 
To ensure proper systemic and gastrointestinal clearance of antibiotics, a 2-day wash-out period was taken into account before the adipose tissue biopsies were collected.

\section{Ex vivo adipocyte lipolysis}

A part of the adipose tissue biopsy $(\sim 500 \mathrm{mg})$ was used for the isolation of mature adipocytes following collagenase digestion in Dulbecco's Modified Eagle's Medium (DMEM)-Ham's $\mathrm{F}_{12}$ at $37^{\circ} \mathrm{C}^{23}$. The resulting suspension was filtered through a $200 \mu \mathrm{m}$ filter and adipocytes were washed once with DMEM-Ham's $F_{12}$ to eliminate collagenase. Isolated mature adipocytes were diluted in DMEMHam's F 12 supplemented with $3 \%$ bovine serum albumin for lipolysis assays and incubated with increasing concentrations of isoprenaline (ISO, a non-selective $\beta$ adrenergic agonist; $10^{-10}-10^{-3} \mathrm{M}$ ) in duplicate at a finale volume of $100 \mu \mathrm{l}$ for $3 \mathrm{~h}$ at $37^{\circ} \mathrm{C}$. Following incubation, $60 \mu \mathrm{l}$ cell-free aliquots of the infranatant were collected for glycerol determination (lipolysis index) using the EnzyChrome ${ }^{\mathrm{TM}}$ Adipolysis assay kit (Gentaur, Eersel, The Netherlands). Glycerol release was expressed per cell number and relative to baseline, as lipolysis index.

\section{Adipose tissue gene expression analyses}

RNA was extracted from frozen AT ( $500 \mathrm{mg})$ using Trizol chloroform extraction (Invitrogen, Cergy Pontoise, France). Next, total RNA (100 ng per sample) was labeled by Whole-Transcript Sense Target Assay and hybridized to human wholegenome Affymetrix Gene 1.1 ST arrays targeting 19793 unique genes (Affymetrix, Santa Clara, CA, USA). Quality control and data analysis have been described in detail previously ${ }^{24}$. Individual genes on the array were defined as changed when comparison of the normalized signal intensities showed a FDRq (false-discovery adjusted $p$-value) $<0.05$ in a 2-tailed paired t-test with Bayesan correction ${ }^{24}$.

\section{Adipose tissue protein expression}

Adipose tissue biopsies (100-300 mg) were ground to a fine powder under liquid nitrogen and homogenized in 200-600 $\mu \mathrm{L}$ radioimmunoprecipitation assay (RIPA) buffer supplemented with a protease/phosphatase inhibitor cocktail (Cell Signalling Technology Europe, Leiden, The Netherlands). Lysates were vortexed for $5 \mathrm{~min}$ at room temperature and centrifuged at $14.000 \mathrm{rpm}$ for $30 \mathrm{~min}$ at $10^{\circ} \mathrm{C}$. Infranatant was carefully transferred to new tubes. Protein concentrations were 
determined using the Pierce ${ }^{\circledR}$ BCA protein assay kit (Santa Cruz Biotechnology Inc., Heidelberg, Germany). Samples were stored at $-80^{\circ} \mathrm{C}$ prior to analysis. $25 \mu \mathrm{g}$ protein was separated on Any kD SDS-PAGE gels (Bio-Rad Laboratories, Veenendaal, The Netherlands) and subsequently blotted semi-dry onto nitrocellulose membrane using Trans-Blot ${ }^{\circledR}$ Turbo Transfer System (Bio-Rad Laboratories, Veenendaal, The Netherlands). Following transfer, membranes were blocked for $1 \mathrm{~h}$ in blocking buffer (Tris-buffered saline with $0.1 \%$ Tween 20 (TBS-T), 5\% nonfat dry milk). The membranes were incubated overnight at $4^{\circ} \mathrm{C}$ with primary antibodies diluted in blocking buffer. Next, membranes were incubated with the corresponding secondary antibodies for $1 \mathrm{~h}$ at room temperature. The primary antibodies used were adipose triglyderide lipase (ATGL) (\#2138), total HSL (\#4107) and Phospho-HSL Ser563 (equivalent to Ser552 of human HSL), a major protein kinase A (PKA) target (\#4139), all from Cell Signalling. $\beta$-actin was used as loading control (Santa Cruz, \#sc-47778, 1:1000 dilution). Secondary antibodies were horse-radish peroxidase-conjugatedIgG $\alpha$-swine or $\alpha$-rabbit (DAKO, Heverlee, Belgium). Antigen-antibody complexes were visualized by chemiluminescence using SuperSignalTM West Femto extended Duration Substrate (Life Technologies, Gent, Belgium). Visualization and analysis was performed using a Chemidoc XRS system (Bio-Rad Laboratories, CA, USA) and Quantity One software.

\section{Calculations and statistical analysis}

The concentration of agonist producing the half-maximum effect (EC50) was determined using logistic conversion of each dose-response curve as described previously ${ }^{25}$. The negative logarithm of the $\mathrm{EC}_{50}$ value $\left(\mathrm{pD}_{2}\right)$ was defined as the $\beta$-adrenergic sensitivity. Analysis of variance (ANOVA) was applied to compare subject characteristics at baseline. Treatment effects within and between groups were tested using repeated-measures ANOVA. A dependent or independent $\mathrm{t}$ test was used

to assess differences before and after treatment and to assess differences between PLA, AMOX or VANCO, respectively. Data are expressed as mean \pm SEM. Statistical analysis was performed using SPSS version 20.0 (Chicago, IL, USA). $P<0.05$ was considered to be statistically significant. 


\section{Results}

\section{Subject characteristics}

Baseline subject characteristics are shown in Table 4.1. No significant differences were present between the PLA and VANCO group and the AMOX and VANCO group, except for a higher insulin sensitivity (homeostasis model assessment for insulin resistance (HOMA-IR) $3.3 \pm 0.4$ vs. $5.0 \pm 0.6, P<0.05$ ) and lower fasting insulin concentrations $(12.3 \pm 1.4$ vs. $18.4 \pm 2.0 \mathrm{mU} / \mathrm{l}, \mathrm{P}<0.05)$ in the PLA versus AMOX group.

Table 4.1. Baseline characteristics

\begin{tabular}{lllll}
\hline & PLA $(\mathrm{n}=15)$ & AMOX $(\mathrm{n}=12)$ & VANCO $(\mathrm{n}=9)$ & P-value \\
\hline Age (years) & $62.7 \pm 1.6$ & $54.3 \pm 3.4$ & $58.7 \pm 2.8$ & 0,068 \\
Body Weight $(\mathrm{kg})$ & $90.5 \pm 3.3$ & $93.7 \pm 2.7$ & $97.8 \pm 3.2$ & 0,429 \\
BMI (kg/m $)$ & $29.5 \pm 0.9$ & $29.6 \pm 1.0$ & $31.7 \pm 0.8$ & 0,238 \\
Fasting glucose $(\mathrm{mmol} / \mathrm{l})$ & $5.9 \pm 0.1$ & $6.0 \pm 0.2$ & $6.0 \pm 0.2$ & 0,919 \\
Fasting Insulin $(\mathrm{mU} / \mathrm{l})$ & $12.5 \pm 1.5^{*}$ & $18.4 \pm 2.0$ & $18.2 \pm 1.6$ & 0,022 \\
HOMA-IR & $3.3 \pm 0.4^{*}$ & $5.0 \pm 0.6$ & $4.8 \pm 0.4$ & 0,028 \\
TAG (mmol/l) & $1.13 \pm 0.11$ & $1.40 \pm 0.22$ & $1.04 \pm 0.17$ & 0.347 \\
FFA (umol/l) & $587.6 \pm 29.2$ & $579.2 \pm 53.8$ & $580.9 \pm 48.5$ & 0.990 \\
\hline
\end{tabular}

Characteristics given as mean \pm SEM. The P-value represents the difference between the 3 groups (one-way ANOVA). *Significant difference between PLA-AMOX (Bonferronipost hoc testing, $\mathrm{P}<0.05)$; BMI: Body mass index; HOMA-IR: Homeostasis model assessment for insulin resistance.

\section{Ex vivo lipolysis}

We investigated the effect of antibiotics treatment on basal and $\beta$-adrenergically mediated lipolysis in freshly isolated mature adipocytes. Basal glycerol release, expressed per number of cells, was not altered following treatment (ANOVA $\mathrm{P}=0.314$, Table 4.2). In addition, maximal ISO-mediated lipolytic response (adjusted for cell number) was comparable between groups ( $P=0.671$, Table 4.2). The half-maximal effective concentration $\left(\mathrm{EC}_{50}\right)$ and $\mathrm{pD} 2\left(-\log \mathrm{EC}_{50}\right)$ values for ISO, which were calculated based on the dose-response curves (Supplementary

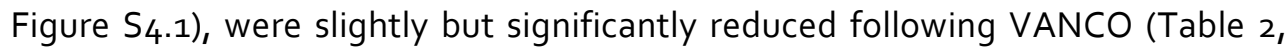
$\mathrm{P}<0.05$ ), indicative of increased $\beta$-adrenergic sensitivity. In line, VANCO induced a modest leftwards shift of the dose-response curve of ISO (Supplementary

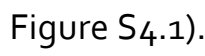


Table 4.2. Basal and maximal ISO-mediated lipolytic response in SCAT adipocytes before and after intervention, compared to placebo

\begin{tabular}{llllll}
\hline & & PLA & AMOX & VANCO & P-value \\
\hline Basal $\left(\mu \mathrm{M} / 10^{-6}\right.$ cells/3h) & pre & $19169 \pm 4114$ & $17879 \pm 4144$ & $19156 \pm 4743$ & 0.314 \\
& post & $18734 \pm 3328$ & $14280 \pm 2542$ & $15275 \pm 2734$ & \\
$\operatorname{Max}\left(\mu \mathrm{M} / 10^{-6}\right.$ cells/3h) & pre & $60274 \pm 9391$ & $88075 \pm 19080$ & $67341 \pm 14669$ & 0.671 \\
& post & $40029 \pm 5182$ & $73856 \pm 21591$ & $49254 \pm 8018$ & \\
EC50 & pre & $2.1 \times 10^{-7} \pm 1.6 \times 10^{-7}$ & $9.1 \times 10^{-7} \pm 3.2 \times 10^{-7}$ & $2.4 \times 10^{-6} \pm 5.7 \times 10^{-7}$ & 0.012 \\
& post & $6.5 \times 10^{-8} \pm 9.5 \times 10^{-8}$ & $6.1 \times 10^{-7} \pm 1.5 \times 10^{-5}$ & $1.0 \times 10^{-6} \pm 5.9 \times 10^{-7} *$ & \\
pD2 & pre & $6.5 \pm 0.3$ & $6.2 \pm 0.3$ & $6.0 \pm 0.3$ & 0.012 \\
& post & $7.1 \pm 0.3$ & $5.8 \pm 0.5$ & $6.7 \pm 0.4 *$ & \\
\hline
\end{tabular}

Values are given as mean \pm standard error of the mean (SEM). P-value represents the overall timextreat $\mathrm{P}$-value of the repeated measures ANOVA. Post-hoc testing was performed using a dependent t-test. * $\mathrm{P}<0.05$ pre vs.post.

\section{$\beta$-adrenoceptor expression and lipase activation}

Next, targeted microarray and Western Blot analyses on adipose tissue biopsies were performed to determine whether the improved $\beta$-adrenoceptor responsiveness following VANCO was related to changes in receptor expression and activation of major lipolytic proteins. The microarray data revealed no significant changes in adrenoceptor expression or post-receptor signaling after VANCO treatment (Table 4.3). In accordance with these findings, total protein content of ATGL, HSL and the phosphorylation status (on Ser563) of HSL were not significantly altered following VANCO treatment (Figure 4.1A-C and

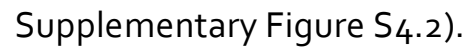

\section{Discussion}

The present study investigated whether short-term antibiotic treatment alters adipose tissue lipolysis in humans. Here, we demonstrated that oral administration of VANCO but not AMOX for 7 days slightly but significantly increased ex vivo $\beta$-adrenergic sensitivity in adipocytes derived from obese insulin resistant men. Surprisingly, however, this was not accompanied by 
changes in adipose tissue $\beta$-adrenoceptor expression or post-receptor events (i.e. HSL phosphorylation). Together, the present data suggest that short-term microbiota manipulation by antibiotics treatment has no pronounced effects on adipose tissue lipolysis in obese men.

We have previously reported that VANCO but not AMOX treatment markedly reduced microbiota diversity and altered its composition, predominantly by decreasing gram-positive Firmicutes ${ }^{21}$. The present findings indicated that VANCO slightly improved $\beta$-adrenoceptor sensitivity as compared to PLA.

This was, however, not accompanied by changes in gene expression of $\beta$ adrenoceptors or lipolytic genes. In line, we did neither observe any changes in downstream signaling, including ATGL and HSL protein content, nor HSLphosphorylation at Ser563 (equivalent to Ser552 of human HSL). However, we cannot exclude effects of VANCO on $\beta$-adrenoceptor responsiveness via catecholamine-mediated phosphorylation of HSL at other serine residues, although this seems unlikely based on the unchanged basal and maximal stimulated lipolysis that we observed in these human adipocytes.

Table 4.3. Fold change of lipolysis-related genes in adipose tissue before and after intervention, compared to placebo

\begin{tabular}{llll}
\hline Gene & Description & AMOX vs. PLA & VANCO vs. PLA \\
\hline PNPLA2 & patatin-like phospholipase domain-containing protein 2 & 1,051 & 1,142 \\
LIPE & hormone sensitive lipase & 1,015 & 1,215 \\
PLIN1 & perilipin 1 & 1,079 & 1,185 \\
ABHD5 & abhydrolase domain containing 5 & $-1,125$ & $-1,181$ \\
GoS2 & Go/G1 switch 2 & 1,075 & 1,164 \\
ADRB1 & adrenoceptor beta 1 & 1,091 & 1,096 \\
ADRB2 & adrenoceptor beta 2 & $-1,035$ & $-1,016$ \\
ADRA2A & adrenoceptor alpha 2A & 1,028 & 1,290 \\
ADRA2B & adrenoceptor alpha 2B & 1,077 & 1,051 \\
ADRA2C & adrenoceptor alpha 2C & $-1,022$ & 1,138 \\
AOP7 & aquaporin 7 & 1,120 & 1,244 \\
\hline
\end{tabular}

Micro-array analysis-derived adipose tissue gene expression for lypolysis-related genes. The mean fold change per gene is expressed as differential fold change compared to placebo (Limma FC for (VANCO_post-VANCO_pre)-(PLA_post-PLA) and: (AMOX_postAMOX_pre)-(PLA_post-PLA_pre). 
Orally administered VANCO is not absorbed into the bloodstream to an appreciable extent as compared to oral intake of AMOX. This suggests that the improved $\beta$-adrenergic responsiveness of adipocyte lipolysis is mainly due to changes in gut microbial-induced signaling rather than an effect of VANCO per $s e^{26}$. We have previously demonstrated that VANCO treatment for 7 days decreased the abundance of Clostridium clusters XIVa and IV, which was accompanied by a reduced conversion of primary to secondary BAs, and a lower concentration of fecal SCFA. Interestingly, SCFA and BA receptors are expressed in adipose tissue, and both SCFA and BA can modulate fasting and $\beta$ adrenoceptor-mediated lipolysis in murine and human adipocytes ${ }^{16,27}$, indicating a possible role for BA and SCFA signaling in catecholamine-induced lipolysis. In addition, ANGPTL4, of which the intestinal expression can be influenced by the gut microbiota, has been suggested to be involved in the lipolytic response to catecholamines in murine adipocytes ${ }^{10}$. However, we have previously shown that VANCO or AMOX did not affect circulating ANGPTL4 concentrations ${ }^{21}$. LPS, derived from Gram-negative bacteria, may also be involved in gut-host signaling ${ }^{28}$. Indeed, LPS has been shown to induce lipolysis in human adipocytes, and stimulated the formation of adrenaline and noradrenaline by macrophages ${ }^{29}$. However, previous data from our group demonstrated that VANCO or AMOX treatment did not affect LPS-binding-protein concentrations. Taken together, gut microbial-induced changes in gut-derived signaling molecules, such as BA and SCFA, might be involved in the improved adrenergic responsiveness of the human ex vivo adipose tissue lipolysis following VANCO treatment.

The slight improvement in VANCO-induced $\beta$-adrenergic sensitivity did not translate in effects on in vivo insulin sensitivity of adipose tissue lipolysis. As previously reported ${ }^{21}$, we found no significant differences in the antibiotic treatment-induced suppression of plasma FFA concentrations during a hyperinsulinemic-euglycemic clamp compared with PLA in these subjects. A limitation of this study is that the gene and protein expression data reflect the total adipose tissue profile rather than cell membrane or lipid droplet-specific expression. Moreover, biopsies were taken after an overnight fast. Therefore, we cannot draw any conclusions regarding changes in adipose tissue lipase activation and translocation of lipid droplet-associated proteins following $\beta$ adrenergic stimulation. Future studies should address underlying mechanisms of microbiota effects on $\beta$-adrenergic sensitivity of lipolysis, in more detail. 
In summary, we demonstrated that short-term manipulation of the gut microbiota by VANCO treatment slightly increased ex vivo $\beta$-adrenergic sensitivity in adipose tissue of obese patients. Future research is needed to establish whether this translates into improved adipose tissue function in the long-term.
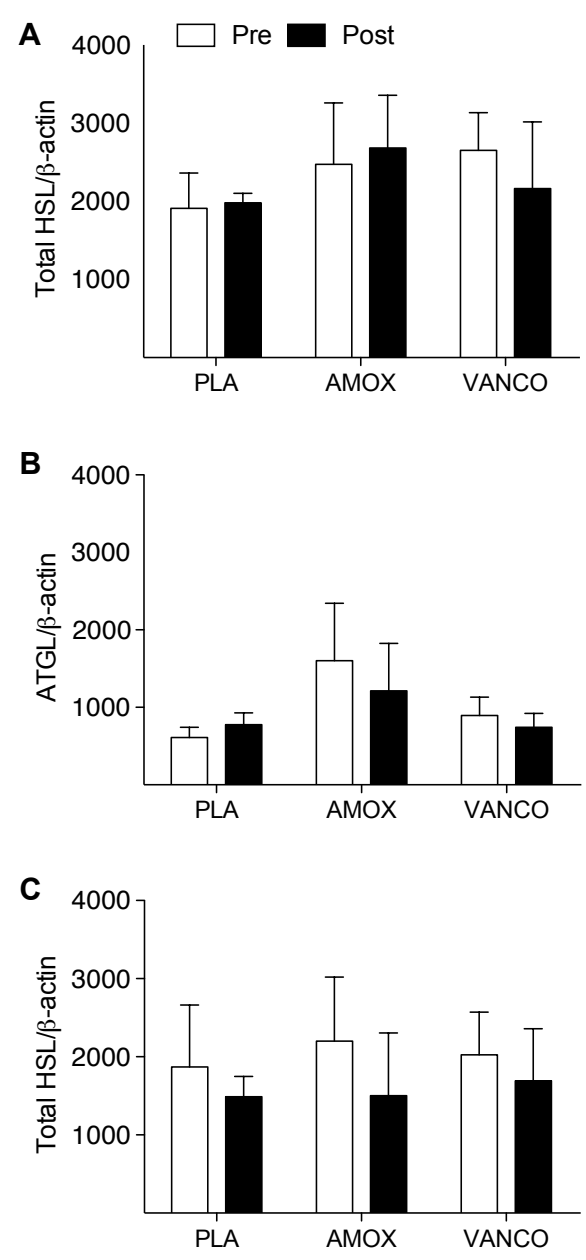

Figure 4.1. Quantitative analysis of the Western blots of HSL (A), ATGL (B) and phosphorylated HSL on Ser563 (equivalent to Ser563 of human HSL) (C). Pre (white bars) and post (black bars) intervention data are normalized for the loading control $\beta$-actin. Values are given as mean $\pm \operatorname{SEM}(n=5$ for PLA, $n=5$ for VANCO and $n=6$ for AMOX). 
Supplemental Figures 

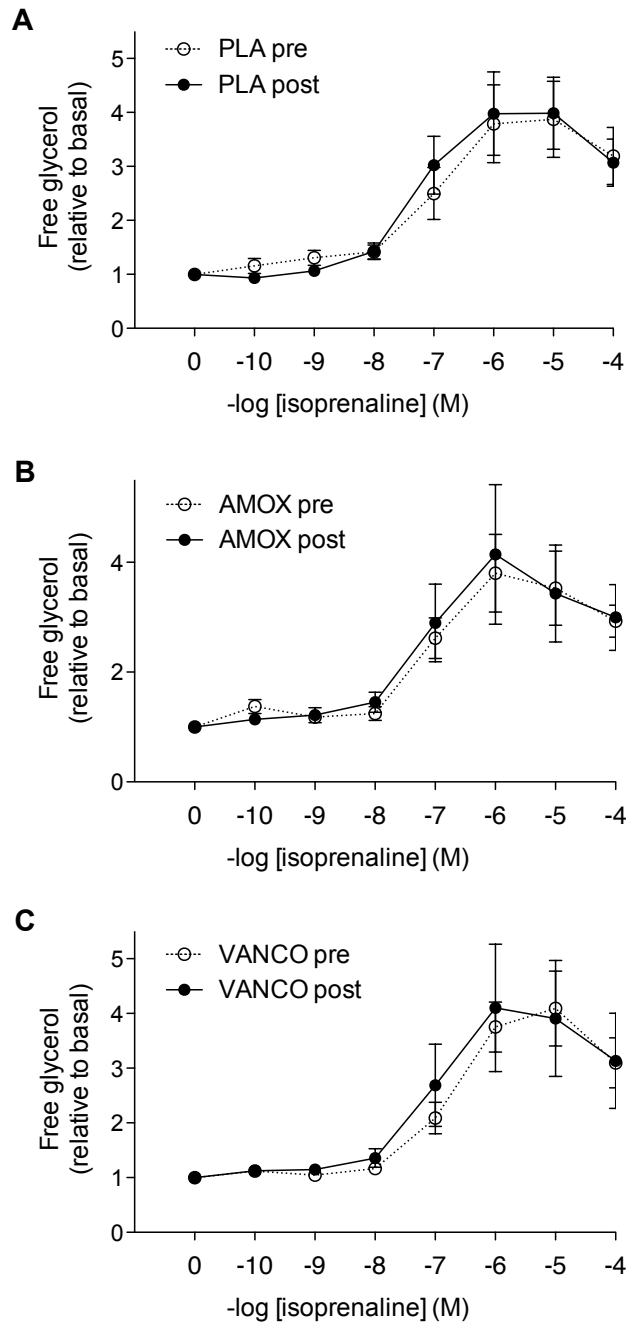

Figure S4.1. Dose-response curves for ISO-mediated lipolytic response in human mature adipocytes derived from the SCAT before and after intervention. Lipolysis (glycerol release in the medium) is expressed compared to baseline, following incubation with increasing concentrations $1 S O\left(10^{-10}\right.$ to $\left.10^{-4} \mathrm{~mol} / \mathrm{l}\right)$ before (circles) and after (triangles) $7 \mathrm{~d}$ treatment with placebo (A), amoxicillin (B) or vancomycin (C). 

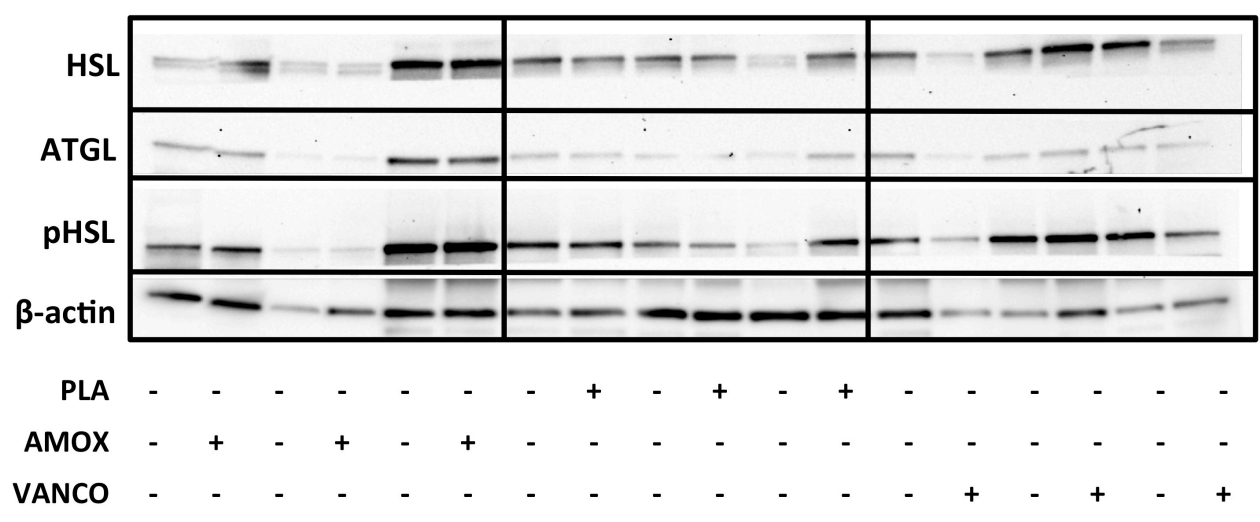

Figure S4.2. Representative Western Blot for lipolytic markers in human adipose tissue. Membranes were probed with antibodies directed against total ATGL, total HSL, phosphorylated HSL (pHSL) on Ser563 (equivalent to Ser563 of human HSL) and $\beta$-actin was used as a loading control. A subset of 3 subjects per group is shown. 


\section{References}

1. Greenhill C. Obesity: Gut microbiota, host genetics and diet interact to affect the risk of developing obesity and the metabolic syndrome. Nature reviews Endocrinology 2015 .

2. Sommer F, Backhed F. The gut microbiota--masters of host development and physiology. Nature reviews Microbiology 2013;11:227-38.

3. Janssen AW, Kersten S. The role of the gut microbiota in metabolic health. FASEB journal : official publication of the Federation of American Societies for Experimental Biology 2015;29:3111-23.

4. Backhed $F$, Ding $H$, Wang $T$, et al. The gut microbiota as an environmental factor that regulates fat storage. Proceedings of the National Academy of Sciences of the United States of America 2004;101:15718-23.

5. Backhed F, Manchester JK, Semenkovich CF, Gordon JI. Mechanisms underlying the resistance to diet-induced obesity in germ-free mice. Proceedings of the National Academy of Sciences of the United States of America 2007;104:979-84.

6. Heimann E, Nyman M, Degerman E. Propionic acid and butyric acid inhibit lipolysis and de novo lipogenesis and increase insulin-stimulated glucose uptake in primary rat adipocytes. Adipocyte 2015;4:81-8.

7. Jocken JW, Langin D, Smit $E$, et al. Adipose triglyceride lipase and hormonesensitive lipase protein expression is decreased in the obese insulin-resistant state. The Journal of clinical endocrinology and metabolism 2007;92:2292-9.

8. Ryden $M$, Jocken J, van Harmelen V, et al. Comparative studies of the role of hormone-sensitive lipase and adipose triglyceride lipase in human fat cell lipolysis. American journal of physiology Endocrinology and metabolism 2007;292:E1847-55.

9. Stinkens $\mathrm{R}$, Goossens $\mathrm{GH}$, Jocken JW, Blaak EE. Targeting fatty acid metabolism to improve glucose metabolism. Obesity reviews : an official journal of the International Association for the Study of Obesity 2015;16:715-57.

10. Gray NE, Lam LN, Yang K, Zhou AY, Koliwad S, Wang JC. Angiopoietin-like 4 (Angpt/4) protein is a physiological mediator of intracellular lipolysis in murine adipocytes. The Journal of biological chemistry 2012;287:8444-56.

11. Staiger $\mathrm{H}$, Haas $\mathrm{C}$, Machann J, et al. Muscle-derived angiopoietin-like protein 4 is induced by fatty acids via peroxisome proliferator-activated receptor (PPAR)delta and is of metabolic relevance in humans. Diabetes 2009;58:579-89.

12. Canfora EE, Jocken JW, Blaak EE. Short-chain fatty acids in control of body weight and insulin sensitivity. Nature reviews Endocrinology 2015;11:577-91.

13. Laurent C, Simoneau C, Marks L, et al. Effect of acetate and propionate on fasting hepatic glucose production in humans. European journal of clinical nutrition 1995;49:484-91. 
14. Fernandes J, Vogt J, Wolever TM. Intravenous acetate elicits a greater free fatty acid rebound in normal than hyperinsulinaemic humans. European journal of clinical nutrition 2012;66:1029-34.

15. Crouse JR, Gerson CD, DeCarli LM, Lieber CS. Role of acetate in the reduction of plasma free fatty acids produced by ethanol in man. Journal of lipid research 1968;9:509-12.

16. Ge H, Li X, Weiszmann J, et al. Activation of $\mathrm{G}$ protein-coupled receptor 43 in adipocytes leads to inhibition of lipolysis and suppression of plasma free fatty acids. Endocrinology 2008;149:4519-26.

17. Aberdein N, Schweizer M, Ball D. Sodium acetate decreases phosphorylation of hormone sensitive lipase in isoproterenol-stimulated $3^{T} 3^{-L 1}$ mature adipocytes. Adipocyte 2014;3:121-5.

18. Abdelkarim $\mathrm{M}$, Caron $\mathrm{S}$, Duhem $\mathrm{C}$, et al. The farnesoid $\mathrm{X}$ receptor regulates adipocyte differentiation and function by promoting peroxisome proliferatoractivated receptor-gamma and interfering with the Wnt/beta-catenin pathways. The Journal of biological chemistry 2010;285:36759-67.

19. Rizzo G, Disante $M$, Mencarelli A, et al. The farnesoid X receptor promotes adipocyte differentiation and regulates adipose cell function in vivo. Molecular pharmacology 2006;70:1164-73.

20. Nogueiras R, Perez-Tilve D, Veyrat-Durebex C, et al. Direct control of peripheral lipid deposition by CNS GLP-1 receptor signaling is mediated by the sympathetic nervous system and blunted in diet-induced obesity. The Journal of neuroscience : the official journal of the Society for Neuroscience 2009;29:591625.

21. Reijnders D, Goossens GH, Hermes GD, et al. Effects of Gut Microbiota Manipulation by Antibiotics on Host Metabolism in Obese Humans: A Randomized Double-Blind Placebo-Controlled Trial. Cell metabolism 2016;24:63-74.

22. Plant Stanol Esters, Focus on intestinal lipoprotein metabolism. Maastricht University Medical Centre+, 2014. at http://www.boxpress.nl/proefschriften/ebooks/els_de_smet/.

23. Jocken JW, Goossens GH, Popeijus H, Essers Y, Hoebers N, Blaak EE. Contribution of lipase deficiency to mitochondrial dysfunction and insulin resistance in hMADS adipocytes. International journal of obesity 2016;40:507-13.

24. Konings $\mathrm{E}$, Timmers $\mathrm{S}$, Boekschoten MV, et al. The effects of 30 days resveratrol supplementation on adipose tissue morphology and gene expression patterns in obese men. International journal of obesity 2014;38:470-3.

25. Ostman J, Arner $\mathrm{P}$, Kimura $\mathrm{H}$, Wahrenberg $\mathrm{H}$, Engfeldt $\mathrm{P}$. Influence of fasting on lipolytic response to adrenergic agonists and on adrenergic receptors in 
subcutaneous adipocytes. European journal of clinical investigation 1984;14:38391.

26. Gonzales M, Pepin J, Frost EH, et al. Faecal pharmacokinetics of orally administered vancomycin in patients with suspected Clostridium difficile infection. BMC infectious diseases 2010;10:363.

27. Al-Lahham SH, Roelofsen $\mathrm{H}$, Priebe $\mathrm{M}$, et al. Regulation of adipokine production in human adipose tissue by propionic acid. European journal of clinical investigation 2010;40:401-7.

28. Cani PD, Bibiloni $R$, Knauf $C$, et al. Changes in gut microbiota control metabolic endotoxemia-induced inflammation in high-fat diet-induced obesity and diabetes in mice. Diabetes 2008;57:1470-81.

29. Zhou J, Yan J, Liang H, Jiang J. Epinephrine enhances the response of macrophages under LPS stimulation. BioMed research international 2014;2014:254686. 
116 


\title{
Microbiota composition is not correlated to insulin sensitivity, but represents distinct alternative dysbiotic states in two independent cohorts of overweight and obese males
}

\author{
D. Reijnders \\ G.D. Hermes \\ R.S. Kootte \\ G.H. Goossens \\ H. Smidt \\ M. Nieuwdorp \\ E.G. Zoetendal \\ E.E. Blaak
}




\section{Abstract}

Background A distinct microbial profile may contribute to differences in metabolic phenotype between lean, obese and obese type 2 diabetic subjects, although results from human studies are inconsistent. Until now, the relationship between microbial patterns and tissue-specific insulin sensitivity has not been investigated in humans.

Aim To examine the relationship between gut microbiota composition at the genus level and tissue-specific insulin sensitivity in overweight and obese humans. Methods Random forest classification and linear and logistic regression analysis were used to investigate the relation between bacterial abundance ( $16 \mathrm{~S}$ ribosomal RNA gene targeted microarray; HITChip) and tissue-specific insulin sensitivity (two-step hyperinsulinemic-euglycemic clamp with $\left[6,6-{ }^{2} \mathrm{H}_{2}\right]$-glucose tracer infusion) in two independent cohorts (cohort $1, n=56$; cohort 2, n=42) of overweight and obese prediabetic men.

Results We did not find a significant relationship between gut microbial composition and tissue-specific insulin sensitivity in either of the cohorts. Cohort 1 showed significant correlations between certain microbial groups and HbA1c. However, these correlations were cohort-specific due to pronounced variation in microbiota composition between both cohorts, suggesting the existence of alternative dysbiotic states in obese, insulin resistant males.

Conclusion Our findings demonstrate no significant relation between gut microbiota composition and tissue-specific insulin sensitivity when the overweight and/or obese state has already developed, and indicate that care should be taken with the extrapolation of data from single cohorts to generalized biological relevance. 


\section{Introduction}

There is increasing evidence to suggest that the gut microbiome is associated with obesity, insulin resistance and diabetes mellitus type 2 (T2DM). Pioneering studies in this field of research demonstrated that germ-free mice had less adiposity, and improved insulin sensitivity and glucose tolerance as compared to conventionalized mice $^{1-4}$. Later studies showed that the gut microbiota composition of lean, obese, and diabetic mice and humans differ ${ }^{5-8}$. Microbial analyses of large genome-wide association studies showed that patients with $\mathrm{T}_{2} \mathrm{DM}$ are characterized by a decrease in the abundance of butyrate-producing bacteria and an increase in opportunistic pathogens ${ }^{5,6,9}$, but data are inconsistent $^{10}$. Discrepancies between studies could be due to the heterogeneity of groups, genetic background, habitual lifestyle, diet and the methodology used to assess the composition and activity of the gut microbiota ${ }^{11}$.

Gut microbiota transplantation studies in rodents and humans have provided evidence for a causal role of the microbiota in adiposity and metabolic health. Fecal transplantation from obese to germ-free mice significantly increased adiposity ${ }^{8,12}$, and fecal transplantation from lean donors to metabolic syndrome patients altered the recipients' microbiota composition with a concomitant minor improvement in peripheral insulin sensitivity ${ }^{13}$. However, the relationship between gut microbiota and tissue-specific insulin action has not yet been established in obese humans.

Here, we aimed to investigate the relationship between the gut microbiota composition and adipose tissue, skeletal muscle and hepatic insulin sensitivity, assessed using the gold-standard two-step hyperinsulinemic-euglycemic clamp with $\left[6,6-{ }^{2} \mathrm{H}_{2}\right]$-glucose tracer infusion, in two independent cohorts of obese men.

\section{Methods}

\section{Study population}

We investigated baseline gut microbiota composition in relation to tissue-specific insulin sensitivity and other indicators of glucose metabolism in two independent cohorts of overweight and obese (BMI $25-45 \mathrm{~kg} / \mathrm{m}^{2}$ ) Caucasian men between 3570 years old (ClinicalTrials.gov NCT02241421 and Dutch Trial Register NTR2705). Cohort 1 (Maastricht) consisted of 56 subjects with impaired fasting glucose levels (glucose concentration $\geq 5.6 \mathrm{mmol} / \mathrm{l}$ ) and/or impaired glucose tolerance (IGT, $2 \mathrm{~h}$ plasma glucose during a $75 \mathrm{~g}$ oral glucose tolerance test $7.8-11.1 \mathrm{mmol} / \mathrm{l}$ ). 
Cohort 2 (Amsterdam) consisted of 42 subjects diagnosed with the metabolic syndrome (according to the NCEP criteria) ${ }^{14}$. Subjects were not allowed to have used antibiotics three months prior to participation. All subjects gave written informed consent before participation after reading the study protocol, which was reviewed and approved by the Local Medical Ethics Committees. All procedures were performed according to the declaration of Helsinki (revised version, 2008, Seoul).

\section{Study design}

Measurements were performed following a 10 h overnight fast. The primary outcome of this study was tissue-specific insulin sensitivity (insulin-mediated glucose disposal (Rd)), hepatic insulin sensitivity (insulin-mediated suppression of endogenous glucose production (\% suppression EGP)), adipose tissue insulin sensitivity (insulin-mediated suppression of plasma free fatty acids (\% suppression FFA)) as determined by a two-step hyperinsulinemic-euglycemic clamp with $\left[6,6-{ }^{2} \mathrm{H}_{2}\right]$-glucose infusion. To this end, one cannula was inserted into the antecubital vein, whereas a second cannula was inserted into a superficial dorsal hand vein to obtain arterialized blood by placing the hand into a hot-box, blowing warm air $\left(5^{\circ} \mathrm{C}\right)$. In the Maastricht cohort (cohort 1 ), after a bolusinjection of $2.4 \mathrm{mg} \mathrm{kg}^{-1}$ was infused, continuous tracer-infusion was started at $0.04 \mathrm{mg} \mathrm{kg}^{-1} \mathrm{~min}^{-1}$ and continued throughout the measurement. After $2 \mathrm{~h}$, lowdose insulin was infused at $10 \mathrm{mU} \mathrm{m}^{-2} \mathrm{~min}^{-1}$ for $2 \mathrm{~h}^{15}$, followed by high-dose insulin at $40 \mathrm{mU} \mathrm{m} \mathrm{min}^{-1}$ for $2 \mathrm{~h}$. By variable co-infusion of a $17.5 \%$-glucose solution, enriched by $1.1 \%\left[6,6-{ }^{2} \mathrm{H}_{2}\right]$-glucose-tracer ${ }^{16}$, plasma concentrations were maintained at $5.0 \mathrm{mmol} / \mathrm{l}$. In the Amsterdam cohort (cohort 2), insulin was infused at $20 \mathrm{mU} \mathrm{m}^{-2} \mathrm{~min}^{-1}$ for $2 \mathrm{~h}$, followed by $60 \mathrm{mU} \mathrm{m}^{-2} \mathrm{~min}^{-1}$ for $2 \mathrm{~h}^{17}$. For calculations of steady-state kinetics, the last 30 minutes of each step $(0,10$ and $40 \mathrm{mU} \mathrm{m} \mathrm{min}^{-1}$ insulin) and the last 20 minutes of each step (o, 20 and $60 \mathrm{mU} \mathrm{m}^{-2}$ $\mathrm{min}^{-1}$ insulin), respectively, were used, and additional blood samples were taken during these time periods.

In addition, we collected fasting plasma samples to determine insulin and glucose concentrations for the calculation of the homeostasis model assessment for insulin resistance (HOMA-IR, [(fasting insulin $(\mu \mathrm{IU} / \mathrm{ml}) \times$ fasting glucose $(\mathrm{mmol} / \mathrm{l})) / 22.5)]$ and $\beta$-cell function (HOMA-B\%, [20 $\times$ (fasting insulin)/(fasting glucose -3.5$)]$. Anthropometrical measurements were performed for the 
calculation of the body mass index (BMI, [weight $(\mathrm{kg}) /$ height $\left.(\mathrm{m})^{2}\right]$ and waist/hip ratio.

\section{Fecal microbiota analysis}

The fecal microbiota composition was determined by analyzing 16S rRNA gene amplicons using the Human Intestinal Tract Chip Microarray (HITChip), a phylogenetic microarray based on 16S rRNA gene sequences of over 1000 intestinal bacterial phylotypes ${ }^{18}$. DNA was isolated from feces using the repeated bead beating method as previously described ${ }^{19}$, and subsequently used for microbiota profiling. In short, 165 rRNA genes were amplified by PCR, followed by in vitro transcription, $\mathrm{C}_{3} / \mathrm{Cy}_{5}$ labelling and fragmentation of RNA, and hybridization. Duplicate hybridizations with a Pearson correlation $>98 \%$ were considered for further analysis, and microbiota profiles were generated by preprocessing of probe-level measurements with min-max normalization and the frozen-RPA probe summarization ${ }^{20}$ into three phylogenetic levels: order-like, genus-like ( $>90 \%$ sequence similarity), and phylotype-like ( $>98 \%$ sequence similarity $)^{18}$. In the present work our analysis focused on the genus-level variation, referred to as species and relatives ('et rel.'). Bacterial groups were considered present and prevalent, when exceeding a HITChip log10 signal of 1.8 in $>20 \%$ of the subjects.

\section{Biochemical analysis}

Blood was collected into pre-chilled tubes, centrifuged at $1000 \mathrm{~g}$, and plasma was snap-frozen and stored at $-80^{\circ} \mathrm{C}$ until analyses. Isotopic enrichment of plasma glucose was determined by electron ionization gas chromatography-mass spectrometry and expressed as tracer-to-tracee ratio for steady-state calculations of rate of disappearance $(\mathrm{Rd})$ and endogenous glucose production (EGP). Plasma glucose and glycerol were determined with the Cobas Fara autoanalyzer, Roche, Switzerland). Plasma insulin was measured with a double antibody radioimunoassay (Millipore, MA, USA) and plasma FFA concentrations were analyzed using standard enzymatic techniques automated on a Cobas Fara auto-analyzer (Roche). 


\section{Statistical analysis}

To determine the bacterial groups whose relative abundance significantly differed between the two cohorts, a non-paired Wilcoxon test was used. We performed linear regression models (unadjusted and adjusting for age) to determine the association of specific genus-like bacterial groups, with the variables under investigation in both cohorts separately. All outcome variables were sorted into quartiles. The highest and lowest range were used for logistic regression and Random Forest classification using the Random Forest and ROCR $\mathrm{R}$ packages ${ }^{21,22}$. Random Forests is a supervised machine-learning technique, which can utilize nonlinear relationships and complex dependencies between genus-like groups to identify bacterial taxa that differentiate the fecal community composition of individuals that are in the highest or lowest range of each host parameter. For all analyses Benjamini-Hochberg correction was applied for multiple testing, and a False Discovery Rate (FDR) of $<10 \%$ (i.e. $q<$ 0.1 ) was considered significant. All analyses were performed in $R, v_{3} \cdot 1.3^{23}$.

\section{Results}

\section{Subject characteristics}

The subject characteristics are shown in Table 5.1. Subjects in both cohorts 1 and 2 were insulin resistant (HOMA-IR: $4.5 \pm 0.2$ and $5.1 \pm 0.3, p=0.13$, respectively). Cohort 1 presented with higher fasting glucose concentrations $(6.1 \pm 0.0 \mathrm{vs}$. $5.8 \pm 0.1 \mathrm{mmol} / \mathrm{l}, \mathrm{p}<0.05)$, whereas insulin concentrations were lower than in cohort $2(16.8 \pm 0.8$ vs. $20.0 \pm 1.2 \mathrm{mU} / \mathrm{l}, \mathrm{p}<0.05)$. Homeostatic model assessment for B-cell function (HOMA-B) was lower in cohort 1 than in cohort $2(136.3 \pm 7.5 \mathrm{vs}$. $192.0 \pm 15.3 \%, p=0.001)$.

Values for peripheral, hepatic and adipose tissue insulin sensitivity in the 2 cohorts are also indicated in Table 5.1. Notably, clamp procedures were slightly different between centers (see Methods). 
Table 5.1. Subject characteristics

\begin{tabular}{|c|c|c|}
\hline & Cohort $1(n=56)$ & Cohort $2(n=42)$ \\
\hline & Maastricht & Amsterdam \\
\hline Age & $59.1 \pm 1.0$ & $54 \cdot 9 \pm 1.1$ \\
\hline Weight (kg) & $96.5 \pm 1.3$ & $116.3 \pm 2.0$ \\
\hline BMI (kg/m2) & $31.2 \pm 0.4$ & $34.8 \pm 0.5$ \\
\hline Waist/hip ratio & $0.95 \pm 0.01$ & $1.05 \pm 0.01$ \\
\hline Fasting insulin (mU/ml) & $16.8 \pm 0.8$ & $20.0 \pm 1.2$ \\
\hline Fasting glucose (mmol/l) & $6.1 \pm 0.1$ & $5.8 \pm 0.1$ \\
\hline HOMA-IR & $4.5 \pm 0.2$ & $5.1 \pm 0.3$ \\
\hline HOMA-B\% & $136 \cdot 3 \pm 7 \cdot 5$ & $192.0 \pm 15 \cdot 3$ \\
\hline $\mathrm{HbA} 1 \mathrm{c}(\%)$ & $5.6 \pm 0.1$ & $5.7 \pm 0.1$ \\
\hline Fasting TAG $(\mathrm{mmol} / \mathrm{l})$ & $1.30 \pm 0.10$ & $1.50 \pm 0.11$ \\
\hline $\mathrm{Rd}(\mathrm{umol} / \mathrm{kg} / \mathrm{min}) *$ & $23.3(10.7-51.4)$ & $26.1(10.1-40.0)$ \\
\hline Suppression EGP (\%)* & $44.1(17.4-79.1)$ & $55.6(30.8-85.0)$ \\
\hline Suppression FFA (\%)* & $45 \cdot 3(-6,1-84 \cdot 1)$ & $74.4(53.9-92.1)$ \\
\hline
\end{tabular}

HOMA-IR: homeostasis model assessement for insulin resistance, HOMA- $\beta \%$ : homeostasis model assessment for $\beta$-cell function, HbA1c: glycated haemoglobin, TAG: triacylglycerol, Rd: rate of disappearance, EGP: endogenous glucose production by the liver, FFA: free fatty acids. Data are expressed as mean \pm SEM. Clamp-results are expressed as mean (range). *due to differences in clamp procedures (see Methods) between centers, no statistical comparison was made between cohorts.

\section{Fecal microbiota composition}

Remarkably, the average microbiota composition in both cohorts showed pronounced differences (Figure 5.1). While Actinobacteria (Bifidobacterium and Propionibacterium) were more abundant in the Maastricht cohort (cohort 1), mainly genus-like groups of Clostridium clusters IV and XIVa had a higher abundance in the Amsterdam cohort (cohort 2). More specifically, Sporobacter termitidis et rel., Papillibacter cinnamivorans et rel., Subdoligranulum variable at rel., Anaerotruncus colihominis et rel. (IV), Butyrivibrio crossotus et rel. and Clostridium symbiosum et rel. (XIVa), of which the latter four are known to contain butyrate-producing species, were more abundant in cohort 2 than in cohort 1 (Figure 5.1). In addition, Uncultered Clostriales I and II had a higher abundance in cohort 2. 


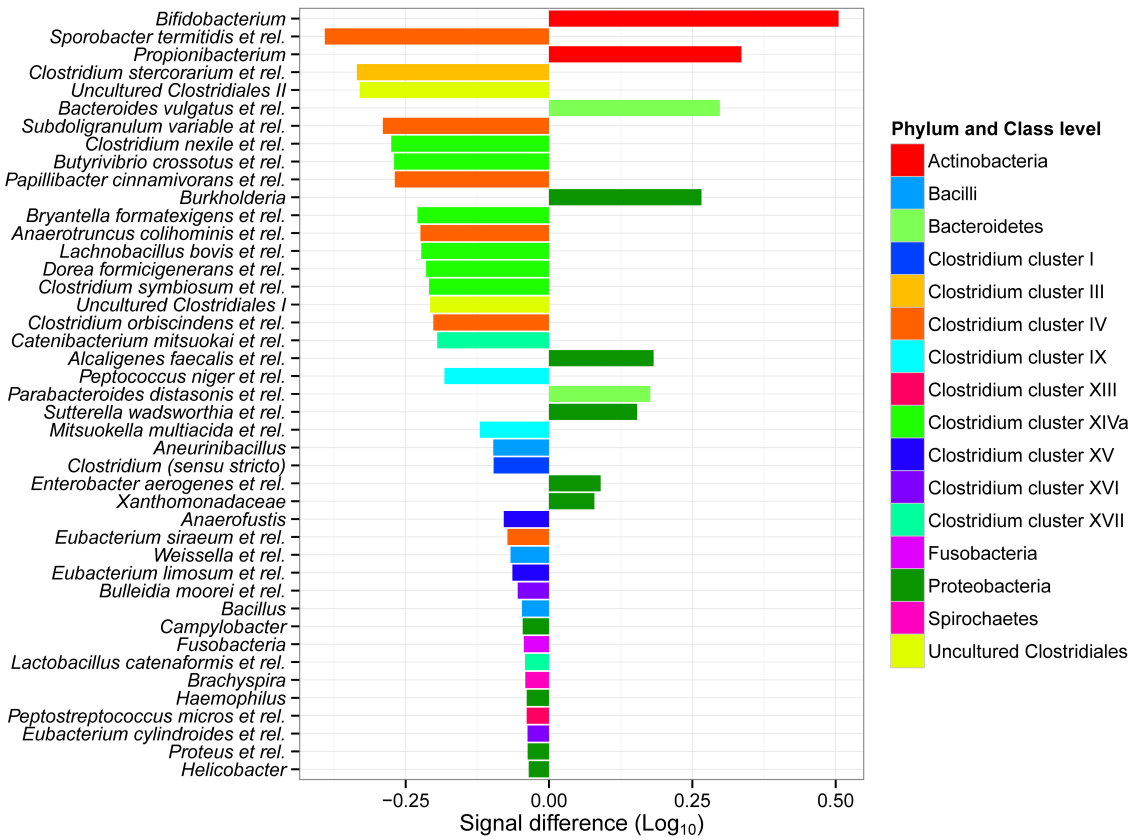

Figure 5.1. Enrichment of bacterial taxa in two separate cohorts of obese men. Genus-like bacterial groups showed significantly different abundance (Log10 signal intensity) between the two cohorts. Left side: taxa enriched in the Amsterdam cohort (cohort 2). Taxa enriched in the Maastricht cohort (cohort 1) are shown on the right. Colors indicate different high level taxa (phylum and class).

\section{Correlations between gut microbiota composition and host metabolic parameters}

\section{Tissue-specific insulin sensitivity}

After correction for multiple testing, peripheral (Rd), hepatic (\% suppression of EGP) and adipose tissue insulin sensitivity (\% suppression of FFA) was not significantly correlated with the abundance of bacterial taxa at the genus-like level in both cohort 1 and cohort 2 (all q>0.85). In addition, we determined nonlinear multivariate relationships between microbiota composition and tissuespecific insulin sensitivity. To this end, we ordered each dataset in quartiles of tissue-specific insulin sensitivity (Rd, \%EGP and \%FFA) and used random forest classifiers to determine the association with the gut bacterial composition by using the highest and lowest quartiles as classes. Both cohorts showed random classification for peripheral, hepatic and adipose tissue insulin sensitivity, 
indicating that there was no significant relationship between gut microbial profiles and tissue-specific insulin sensitivity (data not shown).

\section{Other measures of glucose homeostasis and insulin sensitivity}

Except for a significant correlation between Peptococcus niger et rel. and $\mathrm{HbA} 1 \mathrm{C}$ (Spearman $r=0.57, p=5.52 \mathrm{E}-05, \mathrm{q}=0.06$ ) in cohort 1 , no correlation was found between abundance of specific microbial groups and HOMA-IR, HOMA-B\%, fasting glucose and insulin concentrations in either of the two cohorts (Figure 5.2). Similarly, we did not find any significant correlations between BMI, waist circumference and the abundance of genus-level bacterial groups. Although almost none of the correlations reached statistical significance, it is worthy to note that the pattern of correlations of specific taxa with host variables was distinctly different in both cohorts (Figure 5.2).

Next, we ordered each dataset (cohort 1 and cohort 2) in quartiles of fasting glucose and insulin concentration, HOMA-IR and HOMA-B\%, and used random forest classifiers to determine whether the gut bacterial composition was related to these parameters using the highest and lowest quartiles as classes. Only cohort 1 showed a moderate improvement over a random classification for fasting glucose concentrations and $\mathrm{HbA} 1 \mathrm{C}(\mathrm{AUC}=0.65)$ with Roseburia intestinalis et rel. and Faecalibacterium prausnitzii et rel. as important features of the microbiota.
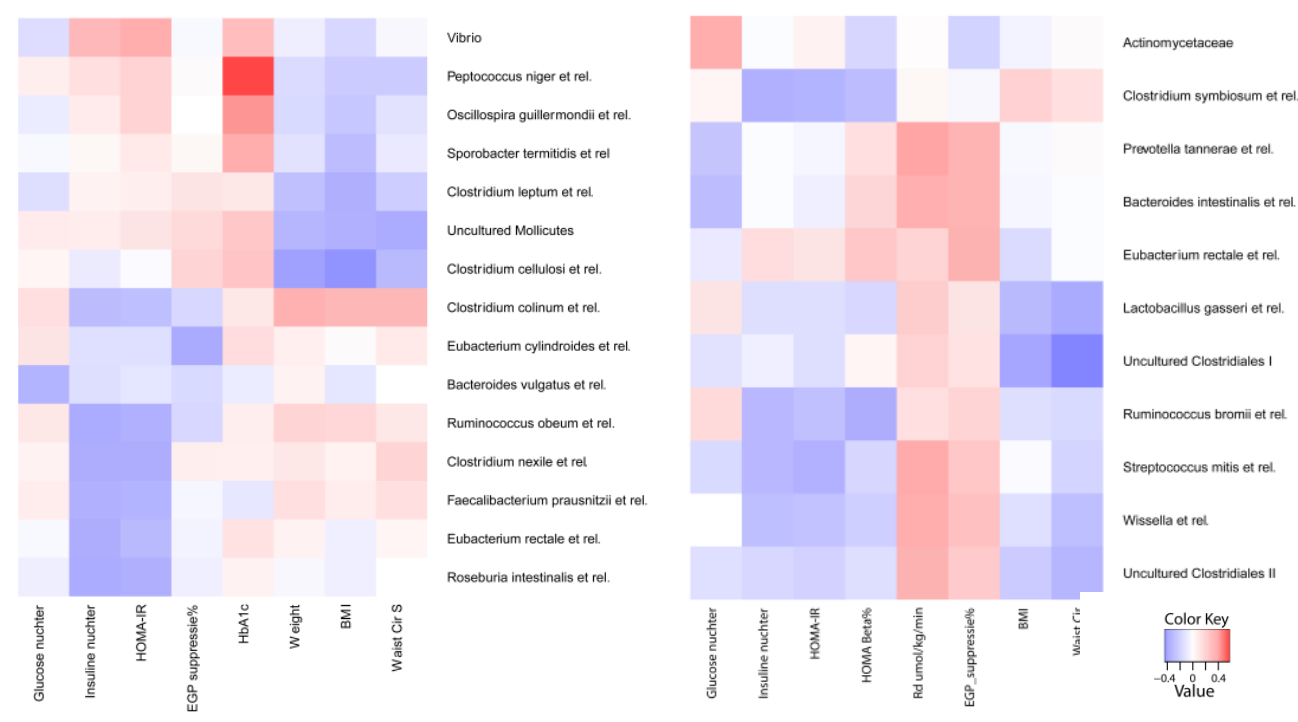
Figure 5.2. Correlation-heatmaps of gut microbiota composition and host metabolic parameters. Heatmaps of Spearman correlations of host metabolic parameters with individual genus like bacterial groups for each cohort separately (left: Maastricht cohort (cohort 1), right: Amsterdam cohort (cohort 2)). Coloring shows direction of the correlation (positive associations, red; inverse associations, blue). Only the positive correlation between Peptococcus niger et rel. and $\mathrm{HbA1c}$ within the Maastricht cohort reached statistical significance. Correlations $>0.35$ are shown.

\section{Discussion}

The aim of the present cross-sectional study was to determine the relationship between gut microbiota composition and tissue-specific insulin sensitivity in two independent cohorts of obese males with a broad range of insulin sensitivity. To our knowledge, this is the first observational study that examined this relationship between gut microbiota composition and peripheral, hepatic and adipose tissue insulin sensitivity, assessed using the gold-standard two-step hyperinsulinemic-euglycemic clamp with $\left[6,6-{ }^{2} \mathrm{H}_{2}\right]$-glucose tracer infusion.

In both cohort 1 and cohort 2, we did not find a significant association between a specific fecal microbial pattern and tissue-specific insulin sensitivity. This is not in line with previous studies indicating a differential microbial composition in impaired glucose tolerant and T2DM subjects as compared to healthy controls $s^{5,6,9,24}$. The present data therefore suggest that gut microbiota composition is not related with insulin resistance when the overweight and/or obese state is established. This may be supported by the findings that fecal transplantation from lean donors to recipients with the metabolic syndrome only slightly improved peripheral insulin sensitivity in a subgroup of subjects, whereas other individuals did not show any effect on these parameters upon the intervention ${ }^{25}$.

Therefore, predicting the role of microbiota in obesity-related insulin resistance is challenging based on exclusively compositional data. Nevertheless, we cannot exclude that the composition of the fecal microbiota may play a role in the worsening of insulin sensitivity in an early stage in the development from a lean towards an overweight/obese phenotype. It is evident that further studies are required to study the fecal microbiota composition in relation to insulin sensitivity in phenotypes varying in adiposity and (tissue-specific) insulin sensitivity. Furthermore, such studies should be extended to also assess 
microbial function.

In line with the findings regarding tissue-specific insulin sensitivity, almost none of the correlations between surrogate (fasting) measures of insulin sensitivity and glycemic control and gut microbiota composition reached statistical significance in either of the two cohorts. Nonetheless, only cohort 1 showed a moderate improvement over a random classification for fasting glucose concentrations and $\mathrm{HbAc1}$ with the butyrate-producers Roseburia intestinalis et rel. and Faecalibacterium prausnitzii as striking features. This is in line with previous studies that showed a decrease in butyrate-producing Firmicutes in $\mathrm{T}_{2} \mathrm{DM}^{5,6}$ and prediabetic humans ${ }^{24}$ as compared to healthy controls. The differences in observations between the two cohorts further illustrates that findings may differ from one cohort to another, which may depend on differences in environment or genetic, microbial or metabolic profile (or interactions between these factors).

Indeed, baseline microbial composition was largely different between cohorts 1 and 2. Additionally, as already indicated above, the pattern of associations between specific taxa and host variables was distinctly different between both cohorts. Compared to cohort 2, the abundance of Bifidobacteria, which has been linked to a healthier phenotype, a reduction in inflammatory markers and an improvement in glucose homeostasis and lipid metabolism in other studies ${ }^{26,27}$, was higher in cohort 1 . Another difference between both cohorts was a lower abundance of specific taxa belonging to Clostridium cluster XIVa, including known butyrate-producing bacteria in cohort 1 (Butyrivibrio crossotus et rel. and Clostridium symbiosum et rel. $)^{28}$.

Currently, we have no clear explanation for these remarkable differences between the two cohorts with respect to gut microbiota composition. Both cohorts showed only slightly different metabolic profiles, were both recruited in the Netherlands ( $220 \mathrm{~km}$ distance). Since previous studies have shown that alternative stable ecosystem states are observed in healthy subjects ${ }^{29}$, the present data suggest that development of such alternative stable states in metabolically compromised subjects is very plausible. This is further supported by comparative microbiota profiling of cohorts 1 and 2 with age and BMImatched metabolically non-compromised obese subjects from other studies present in the HITChip database ${ }^{30}$, which demonstrated that both cohorts had different deviating microbiota compositions (data not shown). This 'alternative stable dysbiotic state' may contribute to inconsistent observations in different 
studies concerning the identification of signature microbes for obesity, inflammatory bowel disease, irritable bowel syndrome and other diseases ${ }^{31-33}$.

\section{Conclusion}

The presented study demonstrated that the abundance of microbial genus-level groups was not significantly related with peripheral, hepatic and adipose tissue insulin sensitivity in two independent cohorts of overweight and obese humans, as determined by state-of-the art clamp methodology. Furthermore, we found pronounced differences in baseline microbiota composition between the two cohorts of metabolically compromised subjects, suggesting the presence of 'alternative stable dysbiotic states', which indicates that care should be taken when translating findings from one single cohort into generalized biological relevance. Nevertheless, these findings do not support a significant relationship between gut microbiota composition and insulin sensitivity when the obese metabolically compromised state has already developed. 


\section{References}

1. Backhed $F$, Ding $H$, Wang $T$, et al. The gut microbiota as an environmental factor that regulates fat storage. Proc Natl Acad Sci U S A 2004;101:15718-23.

2. Backhed F, Manchester JK, Semenkovich CF, Gordon Jl. Mechanisms underlying the resistance to diet-induced obesity in germ-free mice. Proceedings of the National Academy of Sciences of the United States of America 2007;104:979-84.

3. Rabot $S$, Membrez M, Bruneau A, et al. Germ-free $C_{57} B L / 6 J$ mice are resistant to high-fat-diet-induced insulin resistance and have altered cholesterol metabolism. FASEB journal : official publication of the Federation of American Societies for Experimental Biology 2010;24:4948-59.

4. Tremaroli $V$, Backhed F. Functional interactions between the gut microbiota and host metabolism. Nature 2012;489:242-9.

5. Karlsson FH, Tremaroli V, Nookaew I, et al. Gut metagenome in European women with normal, impaired and diabetic glucose control. Nature 2013;498:99103.

6. Larsen N, Vogensen FK, van den Berg FW, et al. Gut microbiota in human adults with type 2 diabetes differs from non-diabetic adults. PloS one 2010;5:e9085.

7. Ley RE, Backhed F, Turnbaugh P, Lozupone CA, Knight RD, Gordon Jl. Obesity alters gut microbial ecology. Proceedings of the National Academy of Sciences of the United States of America 2005;102:11070-5.

8. Turnbaugh PJ, Ley RE, Mahowald MA, Magrini V, Mardis ER, Gordon JI. An obesity-associated gut microbiome with increased capacity for energy harvest. Nature 2006;444:1027-31.

9. Qin J, Li Y, Cai Z, et al. A metagenome-wide association study of gut microbiota in type 2 diabetes. Nature 2012;490:55-60.

10. Schwiertz A, Taras D, Schafer $K$, et al. Microbiota and SCFA in lean and overweight healthy subjects. Obesity 2010;18:190-5.

11. Hermes GD, Zoetendal EG, Smidt H. Molecular ecological tools to decipher the role of our microbial mass in obesity. Beneficial microbes 2015;6:61-81.

12. Ridaura VK, Faith JJ, Rey FE, et al. Gut microbiota from twins discordant for obesity modulate metabolism in mice. Science 2013;341:1241214.

13. Vrieze A, Van Nood E, Holleman F, et al. Transfer of intestinal microbiota from lean donors increases insulin sensitivity in individuals with metabolic syndrome. Gastroenterology 2012;143:913-6 e7.

14. National Cholesterol Education Program Expert Panel on Detection $E_{\text {, }}$ Treatment of High Blood Cholesterol in A. Third Report of the National Cholesterol Education Program (NCEP) Expert Panel on Detection, Evaluation, and Treatment of High Blood Cholesterol in Adults (Adult Treatment Panel III) final report. Circulation 2002;106:3143-421. 
15. Kotronen A, Seppala-Lindroos A, Bergholm R, Yki-Jarvinen H. Tissue specificity of insulin resistance in humans: fat in the liver rather than muscle is associated with features of the metabolic syndrome. Diabetologia 2008;51:130-8.

16. Brehm A, Krssak M, Schmid Al, Nowotny P, Waldhausl W, Roden M. Increased lipid availability impairs insulin-stimulated ATP synthesis in human skeletal muscle. Diabetes 2006;55:136-40.

17. ter Horst KW, Gilijamse PW, Koopman KE, et al. Insulin resistance in obesity can be reliably identified from fasting plasma insulin. International journal of obesity 2015;39:1703-9.

18. Rajilić-Stojanović M, Heilig HG, Molenaar D, et al. Development and application of the human intestinal tract chip, a phylogenetic microarray: analysis of universally conserved phylotypes in the abundant microbiota of young and elderly adults. Environmental microbiology 2009;11:1736-51.

19. Salonen A, Nikkilä J, Jalanka-Tuovinen J, et al. Comparative analysis of fecal DNA extraction methods with phylogenetic microarray: effective recovery of bacterial and archaeal DNA using mechanical cell lysis. Journal of microbiological methods 2010;81:127-34.

20. Lahti L, Elo LL, Aittokallio T, Kaski S. Probabilistic analysis of probe reliability in differential gene expression studies with short oligonucleotide arrays. IEEE/ACM transactions on computational biology and bioinformatics / IEEE, ACM 2011;8:217-25.

21. Liaw AW, M. Classification and regression by randomForest. R News 2002;2:1822.

22. Sing $T$, Sander O, Beerenwinkel N, Lengaver T. ROCR: visualizing classifier performance in R. Bioinformatics 2005;21:3940-1.

23. R: A language and environment for statistical computing. R Foundation for Statistical Computing, 2015. at https://http://www.r-project.org/.)

24. Zhang $X$, Shen $D$, Fang $Z$, et al. Human gut microbiota changes reveal the progression of glucose intolerance. PloS one 2013;8:e71108.

25. Vrieze A, de Groot PF, Kootte RS, Knaapen M, van Nood E, Nieuwdorp M. Fecal transplant: a safe and sustainable clinical therapy for restoring intestinal microbial balance in human disease? Best practice \& research Clinical gastroenterology 2013;27:127-37.

26. Dewulf EM, Cani PD, Claus SP, et al. Insight into the prebiotic concept: lessons from an exploratory, double blind intervention study with inulin-type fructans in obese women. Gut 2013;62:1112-21.

27. Vulevic J, Juric A, Tzortzis G, Gibson GR. A mixture of transgalactooligosaccharides reduces markers of metabolic syndrome and modulates 
the fecal microbiota and immune function of overweight adults. The Journal of nutrition 2013;143:324-31.

28. Barcenilla A, Pryde SE, Martin JC, et al. Phylogenetic relationships of butyrateproducing bacteria from the human gut. Applied and environmental microbiology 2000;66:1654-61.

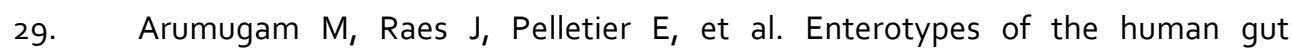
microbiome. Nature 2011;473:174-80.

30. Lahti L, Salojarvi J, Salonen A, Scheffer M, de Vos WM. Tipping elements in the human intestinal ecosystem. Nature communications 2014;5:4344.

31. Ghoshal UC, Shukla R, Ghoshal U, Gwee KA, Ng SC, Quigley EM. The gut microbiota and irritable bowel syndrome: friend or foe? International journal of inflammation 2012;2012:151085.

32. Rajilic-Stojanovic M, Biagi E, Heilig HG, et al. Global and deep molecular analysis of microbiota signatures in fecal samples from patients with irritable bowel syndrome. Gastroenterology 2011;141:1792-801.

33. Wang ZK, Yang YS, Chen Y, Yuan J, Sun G, Peng LH. Intestinal microbiota pathogenesis and fecal microbiota transplantation for inflammatory bowel disease. World journal of gastroenterology 2014;20:14805-20. 
132 


\section{CHAPTER 6}

General Discussion 
Obesity and its metabolic consequences are major contributors to morbidity and mortality rates worldwide ${ }^{1,2}$. Recent studies suggest that alterations in the gut microbiota composition may be one of the underlying mechanisms. Several years ago, the lack of anaerobic culturing systems hampered detailed insight into the bacterial composition. Due to technical advances, the bacterial abundance can now be determined at species level, and the field of microbiota research has since then rapidly gained interest. It were the early studies by Backhed ${ }^{3 / 4}, \mathrm{Cani}^{5}$ and Turnbaugh ${ }^{6}$, which showed for the first time that, in mice, the microbiota composition was associated with adiposity. Additional studies in rodents ${ }^{7-10}$, horses $^{11}$, piglets $^{12}$ and even zebrafish ${ }^{13}$ and fruit flies $^{14}$ provided several hypotheses related to the importance of microbial populations on obesity outcomes. More recently, large metagenomic studies in humans have also indicated that different gut microbial populations may have differential effects on host adiposity and insulin sensitivity ${ }^{15-17}$. The gut microbiota may be of importance in the metabolism of nutrients beyond host-dependent metabolism ${ }^{18,19}$, for the uptake of micronutrients ${ }^{20}$ and in the host metabolic and inflammatory phenotype $\mathrm{s}^{51-24}$. Therefore, interference with a resilient microbiome might alter concentrations of bacterial products such as short chain fatty acids (SCFA), bile acids (BA), amino acids (AA), lipopolysaccharides (LPS) as well as gut hormones (e.g. incretins), inflammatory markers and cytokines. These molecules might affect the gut-brain axis and the cross-talk between the gut, adipose tissue, skeletal muscle, liver and pancreas ${ }^{25,26}$, affecting thereby substrate and energy metabolism. However, human studies that have investigated the contribution of the microbiome to body weight control and insulin resistance are scarce, and underlying mechanisms have not been elucidated yet.

Therefore, the present thesis aimed to address the role of the human gut microbiota in host metabolism. More specific, we determined the effects of gut microbial manipulations using antibiotics on tissue-specific insulin sensitivity and metabolic profile (Chapter $\mathbf{2}$ ) and on host adipose tissue (Chapters $\mathbf{2}$ and $\mathbf{4}$ ), liver (Chapter 2) and skeletal muscle metabolism (Chapters $\mathbf{2}$ and $\mathbf{3}$ ). In addition, in a cross-sectional analysis using two cohorts of obese men we have determined the association between gut microbial species and tissue specific insulin sensitivity and glucose homeostasis (Chapter 5). 


\section{Manipulation of the gut microbiota - an antibiotics approach}

The resilient bacterial populations in the gut are prone to environmental pertubations. Although the microbiota composition is generally stable over a long period during adulthood, fluctuations may occur due to changes in diet, particularly the amount of fat and fiber, medication, the intake of probiotics and disease state ${ }^{27}$. To investigate whether the microbiota is involved in metabolic processes of the host, we intended to temporarily modulate the gut microbiota composition in a robust way and examined the effects on tissue-specific insulin sensitivity, energy and substrate metabolism and the inflammatory profile of the host after the intervention. As a proof-of-concept, we used antibiotics to induce pronounced changes in the bacterial composition. Based on pharmacokinetic and pharmacodynamic properties, and taking into account medical-ethical aspects (e.g. chance of side-effects and antibiotics resistance), we decided to use a regular used dose of vancomycin (VANCO) and amoxicillin (AMOX) in our study (seven days, $1500 \mathrm{mg} /$ day). VANCO, which is not absorbed in the gut and as such does not exert direct systemic effects, is directed against Gram-postive bacteria ${ }^{28}$. Indeed, in Chapter 2, we found that VANCO treatment decreased microbial diversity, with a decrease of the relative abundance of Gram-positive bacteria of the Firmicutes phylum. Among the most strongly affected groups were butyrateproducing species (e.g. Faecalibacterium prausnitzii) as well as species involved in BA dehydroxylation, such as Clostridium leptum. Indeed, fecal and plasma concentrations of butyrate and secondary BA decreased after VANCO. Furthermore, VANCO decreased acetate, caproate and valerate concentrations in feces, whereas primary BA were increased. Noteworthy, we found a compensatory increase of the abundance of some Gram-negative Proteobacteria, members of Clostridium cluster IX and VANCO-resistant Grampositive bacilli.

AMOX is a broad-spectum antibiotic, targeting both Gram-positive and Gramnegative bacteria. AMOX is distributed systemically and, therefore, may have direct effects on peripheral organs. To exclude these systemic effects, we applied a two day wash-out period. Strikingly, in our study neither microbial diversity nor microbial metabolites were significantly affected by AMOX. In line, a previous study also showed no marked microbial changes after AMOX treatment ${ }^{29}$. Nevertheless, mild to moderate effects of AMOX with or without clavulanic acid on the normal microbiota has previously been shown ${ }^{30}$. Our findings, however, 
do not exclude that total bacterial numbers decreased, since HIT-chip methodology provides insight in relative but not absolute changes of specific species. In order to obtain a more complete overview of the bacterial changes in the gut, PCR techniques should be additionally used to determine the amount of bacteria present in the intestine after intervention.

\section{Microbiota manipulation and effects on host metabolism Effects on insulin sensitivity}

In Chapter 2, we investigated whether antibiotic-induced modification of the gut microbiota may affect tissue-specific insulin sensitivity. However, we did not find effects of antibiotics-treatment on peripheral insulin sensitivity, hepatic insulin sensitivity, and adipose tissue insulin sensitivity. Our data are in contrast with rodent studies, and again highlights that rodent data cannot always be extrapolated to humans. In these animal studies, the manipulation of the microbiota composition has been indicated to alter insulin sensitivity ${ }^{7-9,31-34}$. For example, Backhed et al. ${ }^{3}$ showed a $40 \%$ decrease of insulin sensitivity after only two weeks colonization of germ-free mice with gut microbiota. However, a more recent study showed that VANCO-treated mice had little weight change and no improvement in glycemic control ${ }^{35}$.

Furthermore, our data are in contrast to a previous relatively small study in humans ( $n=8$ per group), showing that VANCO induced a slight but significant reduction in peripheral insulin sensitivity ${ }^{29}$. The reason for this apparent discrepancy may be explained by the fact that in the latter study, results were based on a within group comparison (post-treatment versus pre-treatment) since no placebo-group was included in the study design. Moreover, the VANCOinduced decrease in peripheral insulin sensitivity was very modest $(\sim 4 \%)$ and might not be clinically relevant. Additionally, a more recent trial showed that four days of treatment with a broad-spectrum antibiotic cocktail did not affect postprandial glucose metabolism in lean healthy men $^{36}$. Taken together, these and our data clearly demonstrate that in a physiological setting, pronounced microbiota alterations and alterations in SCFA and BA metabolism evoked by antibiotics, do not affect insulin sensitivity in humans.

In line with this, in a cross-sectional analysis of two seperate cohorts of obese, insulin resistant men, we were not able to demonstrate a microbial profile that was associated with tissue-specific sensitivity (Chapter 5 ). This is in contrast with 
large metagenome-wide association studies, which have shown that obese individuals and patients with $\mathrm{T}_{2} \mathrm{DM}$ or insulin resistance had decreased intestinal microbiota diversity ${ }^{15-17,37}$. More specific, lower Firmicutes and higher Proteobacteria abundances in $\mathrm{T}_{2} \mathrm{DM}^{6,15,16,38}$ and prediabetic humans ${ }^{37}$ as compared to healthy controls have been described. Importantly, these associations have mostly been based on fasting values, whereas the relationship between gut microbiota and insulin action, as determined by a hyperinsulinemiceuglycemic clamp as we have employed, has never been established in obesity before. Notably, additional analysis of our cohorts regarding fasting parameters also did not show a significant correlation between the gut microbiota composition and glucose homeostasis. Therefore, our data do not support a major role of the gut microbiota in glucose homeostasis and insulin sensitivity when the obese state has already developed.

\section{Effects on energy regulation, inflammation, hormones and metabolites}

In addition to effects on insulin sensitivity, we investigated the effect of the antibiotics-intervention on mechanisms that have been suggested to underly the microbiota-insulin sensitivity relationship. As mentioned above, we found that VANCO treatment for seven days markedly changed SCFA and BA profiles, whereas no effects of AMOX on these parameters were found. The alterations of SCFA and BA profiles did not affect fasting plasma glucose or insulin levels, postprandial glucose tolerance, and plasma concentrations of (gut) hormones and metabolites. Strikingly, we did not find any significant impact of antibiotic treatment on energy harvesting, whole-body energy expenditure and substrate oxidation. In contrast to animal studies ${ }^{6,38}$, we found a lower energy density ( $\mathrm{kcal} / \mathrm{g}$ feces) in fecal samples after VANCO treatment, suggesting increased energy harvest. Importantly, however, VANCO treatment yielded a higher fecal weight, resulting in a similar total amount of calories excreted via the feces per day between groups.

It has been indicated that the reduction of SCFA, deconjugated BA and secondary $B A$ concentrations may interfere with the host immune response through a reduced activation of the GPR 43 on macrophages and monocytes ${ }^{39,40}$, reduced activation of the anti-inflammatory $T_{\text {reg }}$ network ${ }^{21,41,42}$ and increased innate immune cell activity ${ }^{23,43}$. The decrease in secondary BA and butyrate after VANCO did, however, not result in increased systemic inflammation in our study. 
In line, no effects of AMOX and VANCO on gut permeability, circulating LPSbinding protein and pro-inflammatory cytokine concentrations were found. Our results are in contrast with rodent studies, showing that (prebiotic) microbial manipulation improved ${ }^{44}$, whereas antibiotics reduced gut epithelial integrity ${ }^{5}$. Proteobacteria have been suggested to have properties that can directly exacerbate inflammation and (GI)-tissue damage ${ }^{45-47}$, whereas butyrate may conserve the intestinal barrier and decrease gut permeability by modulating the expression of tight junction protein and mucins ${ }^{48,49}$. Our results demonstrated that the abundance of Proteobacteria increased and butyrate concentations decreased by VANCO treatment. We cannot exclude that the apparent opposite effects of Proteobacteria and butyrate on inflammation may have counteracted each other to some extent, and might therefore underlie the absence of any effects on gut permeability and systemic concentrations of inflammatory markers. Moreover, since obese subjects may already have an impaired GI barrier function ${ }^{50,51}$, it might be that the antibiotic-related dysbiosis has no substantial additive effect on gut permeability in our study population.

\section{Microbiota manipulation - effects on the long term?}

Even though antibiotics are widely used in the clinical setting, the exact longterm effects on commensal bacterial populations and microbial community resilience are largely unknown ${ }^{27,52}$. In agreement with observations that some populations of bacteria do not immediately or completely recover after antibiotic treatment ${ }^{52,53}$, we found some microbiota populations in the VANCO-treated group to be still deviant from baseline after eight weeks follow-up. Most bacterial groups that increased in abundance immediately after seven days antibiotic treatment (e.g. Proteobacteria) had returned to baseline levels, but several members of Clostridium clusters IV and XIVa were still decreased in some individuals at the end of follow-up.

Also in animal studies, VANCO evoked a pronounced effect on Clostridium leptum and Clostridium coccoides clusters, which had recovered to a large extent twothree days post-treatment ${ }^{10}$. However, in the latter study, levels of some metabolites did not recover immediately after the cessation of antibiotic treatment. Therefore, in our study, we have determined fasting insulin and glucose concentrations (to calculate HOMA-IR), as well as adipocyte size and fecal energy harvest eight weeks after treatment cessation. Strikingly, we 
demonstrated that whole-body insulin sensitivity (HOMA-IR), adipocyte size and fecal energy did neither alter immediately following seven days antibiotic treatment, nor after eight weeks follow-up, even though the microbiota composition in the VANCO-treated group had not completely recovered at the end of follow-up. In agreement with our findings, it has recently been shown a four-day treatment with an antibiotic cocktail in lean healthy humans did not cause short (four days) or long-term (180 days) changes in glucose tolerance ${ }^{36}$. It is, however, tempting to postulate that it takes a longer and more persistent manipulation of the microbiome before alterations in host metabolism become evident. For example, the expression of SCFA and BA receptors, tight-junction proteins in the gut and the inflammatory response might need some time to adapt to different bacterial concentrations and their metabolites. Most animal studies that have demonstrated a large impact of antibiotic use on metabolic health outcomes, exposed these animals to antibiotics in their drinking water for at least two-four weeks ${ }^{57,8}$. In addition, studies in humans that have shown a relation between antibiotic use and adiposity also found this effect when subjects were exposed to a more prolonged treatment (four-six weeks) with a higher dosage of antibiotics, or a combination of antibiotics. ${ }^{54,55}$ Although day-to-day variations in microbiota composition have been reported, the adult microbiota is in general stable over time, with a certain resilience that decreases during the ageing proces ${ }^{27}$. The fact that we do not find any metabolic effects, except for adipose tissue (AT) gene expression, shows that human metabolism is not affected following short-term alterations in the microbiome, nor after a prolonged time period in which the microbiome has not returned to the baseline composition (eight wk follow-up).

Nevertheless, it is important to emphasize that the present study does not fully exclude a role for the gut microbiota in host metabolism. Since we studied obese, insulin resistant men with impaired glucose metabolism, we cannot exclude that microbiota manipulation by antibiotics may have more pronounced effects in women or less metabolically compromised individuals.

Furthermore, the extent to which antibiotics affect the gut microbiota composition and host metabolism might be largely dependent on the subjects' long-term dietary habits. Dietary composition (especially fat and fiber content) has been found to be extremely important in shaping the microbiome ${ }^{56,57}$. Therefore, enriching a diet with prebiotics, such as resistant starch or 
oligosaccharides for a long period of time might be an effective way to alter host metabolism in obese insulin resistant subjects ${ }^{58,59}$. Moreover, this might be a relatively easy and physiologically relevant way to investigate long-term manipulation of the microbiome. Supplementing the diet with probiotics, which have been linked to positive health outcomes, is an alternative to study longterm effects on metabolism.

\section{Microbiota manipulation - effects on adipose tissue metabolism.}

In order to obtain a more detailed insight into mechanims that may provide a link between gut microbiota and metabolism, we investigated the effects of antibiotics specific for AT function. First, in Chapter 2, we investigated the effect of seven days antibiotics on AT gene expression (microarray analysis). VANCO, but not AMOX treatment, significantly decreased immunity-related gene sets in AT. The fact that we did not find this downregulation after AMOX treatment, suggests that the VANCO-induced alterations in microbiota metabolites (e.g. SCFA, BA) might have played a role. However, these gene expression alterations did not translate into changes in systemic low-grade inflammatory markers, including TNF- $\alpha$, IL- 6 and IL-8) Both VANCO and AMOX increased AT gene expression of pathways related to oxidative metabolism (TCA cycle, oxidative phosphorylation) and pathways feeding into these cycles (glycolysis, betaoxidation) as compared to placebo. Noteworthy, the VANCO-treated subjects showed this upregulation of genes related to oxidative metabolism pathways independently of changes in the placebo group, whereas AMOX-induced effects on these genes were due to a decrease observed in the placebo-group. Mitochondrial ATP synthesis in mature white adipocytes is essential for major metabolic pathways such as lipolysis, de novo fatty acid synthesis, triacylglycerol (TAG) synthesis, glyceroneogenesis and the re-esterification of fatty acids ${ }^{60}$. However, in Chapter 4 we demonstrated that antibiotic treatment had no significant effects on ex vivo basal lipolysis and $\beta$-adenergic stimulation of lipolysis in mature adipocytes. In agreement with these functional lipolysis data, $\beta$-adrenoceptor expression and post-receptor signaling pathways were unaltered following antibiotics treatment. This is in line with unchanged adipose tissue insulin sensitivity, as determined in vivo by the insulin-mediated suppression of free fatty acid concentrations during the hyperinsulinemic-euglycemic clamp 
(Chapter 2). Interestingly, it has previously been found that fasting and $\beta$ adrenoceptor-mediated lipolysis in murine and human adipocytes is modulated by SCFA and $\mathrm{BA}^{61,62}$. Moreover, human studies have shown that oral or rectal administration of SCFA, in particular acetate, significantly decreased plasma FFA concentrations ${ }^{63-65}$. The reduction in concentrations of SCFA and BA metabolites in our study might not have been large enough to induce alterations in the lipolytic response in AT. Multiple other mechanisms have been suggested to affect adipose tissue lipid turnover, including an altered production of angiopoeitin-like 4 (ANGPTL4), lipopolysaccharide (LPS) and incretins ${ }^{66}$. However, plasma concentrations of these factors were not altered by antibiotics in our study. Interestingly, the adipocyte $\beta$-adrenergic sensitivity was slightly but significantly improved after VANCO treatment, although the physiological importance of this slight effect remains to be determined.

Finally, as described in Chapter 2, we investigated AT morphology since abdominal subcutaneous adipocyte size is tightly associated with AT function and insulin resistance ${ }^{67,68}$. Adipocyte hypertrophy, as frequently observed in obese individuals, is correlated with AT inflammation and impairments in lipid metabolism ${ }^{67,68}$. The gut microbiota imbalance that may be present in obesity is considered as an important trigger of white adipose tissue (WAT) plasticity via the regulation of energy harvesting ${ }^{69}$ and fat storage ${ }^{3}$ in animals. We did not find any changes in adipose tissue morphology, neither in mean adipocyte size nor in the proportion of small and large adipocytes. In agreement with unchanged adipocyte morphology, we did not find changes in circulating concentrations of leptin, an adipokine that is secreted from subcutaneous adipose tissue in proportion to adipocyte size ${ }^{70}$. Interestingly, this indicates that the VANCOinduced effects on AT gene expression of pathways related to oxidative metabolism and inflammation, as discussed above, are independent of changes in adipocyte size. 


\section{Microbiota manipulation - effects on skeletal muscle metabolism}

The skeletal muscle accounts for approximately $80 \%$ of insulin-stimulated glucose disposal and, therefore, plays a prominent role in development and progression of insulin resistance and type 2 diabetes mellitus ${ }^{71}$. In Chapter 3, we investigated the effects of modulation of the microbiota composition by antibiotics on postprandial insulin sensitivity and skeletal muscle substrate metabolism under fasting and postprandial conditions. Interestingly, postprandial glucose and insulin concentrations and forearm glucose uptake after the intake of a high-fat mixed-meal did not change following VANCO and AMOX treatment. Additionally, we found that antibiotics treatment did neither change fasting and postprandial TAG and FFA concentrations, nor the extraction of TAG and FFA across the forearm muscle. Finally, no changes in lactate flux across forearm muscle were observed. These findings clearly indicate that antibiotics treatment did not significantly affect human forearm muscle metabolism.

Alterations in glucagon-like peptide-1 (GLP-1) and SCFA concentrations have been suggested to underlie changes in skeletal muscle fat oxidation and substrate metabolism ${ }^{72,73}$ through metabolic pathways involving the phosporylation of $\mathrm{AMPK}^{4}$ or effects on microvascular blood flow ${ }^{18}$. Furthermore, SCFA have been suggested to affect peripheral substrate metabolism, since their receptors (GPR41 and 43) are expressed in skeletal muscle ${ }^{7475}$. Although circulating butyrate concentrations were altered after VANCO treatment, this did not alter fasting and postprandial whole-body energy expenditure, fat and carbohydrate oxidation, as described in Chapter 2. Thus, although several lines of evidence suggest that the gut microbiota-skeletal muscle cross-talk is involved in substrate metabolism and insulin sensitvity in rodents ${ }^{25,76}$, the contribution of AMKP, GLP-1, SCFA and other factors in the microbiota-muscle interaction is not supported by our antibiotics study, and requires more investigation.

\section{Conclusion}

Our data, showing pronounced modulation of microbial composition but no effects on host metabolism, are not in line with many data derived from animal experiments and a few other human studies. VANCO treatment for seven days 
markedly changed the composition of the gut microbiota, with consequent alterations of SCFA and BA profiles. However, these alterations did not affect tissue-specific insulin sensitivity, energy harvest, energy expenditure and substrate metabolism, gut permeability, adipocyte morphology and plasma concentrations of hormones, metabolites and low-grade inflammatory markers. Even after eight weeks follow-up, when the microbiota compostion was still considerably altered, no differences in energy harvesting, adipocyte size and whole-body insulin sensitivity (HOMA-IR) were found.

The fact that we were not able to confirm the clear effects observed in animal studies shows, once again, that the human organism and its interaction with the environment is extremely complex, and mice and man are not alike. Interindividual differences in lifestyle, diet, country of origin, mode of delivery at birth, medical history, travel habits and cultural habits make it hard to determine the exact role of the microbiome in obesity and comorbities, and hamper comparisons of outcomes between studies. Nevertheless, human data thus far does not provide robust evidence that the gut microbiota has a pronounced impact on host metabolism. Long term, moderate alterations of the microbiota composition (e.g. by prebiotics or probiotics or altering diet composition), might be needed to obtain health benefits. Whether this 'slow and steady' approach wins the race needs to be confirmed in large, well-controlled human trials.

\section{Future perspectives}

Based on the outcomes of the present work, as extensively described in this thesis, together with available evidence in literature, several suggestions for future studies can be made:

1. It is important that future studies include both men and women in a study protocol that aims to investigate metabolism. Gender differences in physiology and sex hormones alter the susceptibility to interventioneffects. Moreover, recent studies have shown that fecal microbiota composition might differ between men and women. The gut microbiome in women was characterized by a lower abundance of Bacteroidetes than men $^{77,78}$, and it has been shown that the response to dietary fibers differ between males and females ${ }^{77,79}$. Therapies to treat microbiota dysbiosis by for example prebiotics, might therfore not work equally well for both sexes. In addition, the relationship between microbiota and BMI has been 
found in women but not in men $^{77}$, whereas the relationship between peripheral insulin sensitivity and the microbiome was only found in men (unpublished data from our group).

2. The large inter-individual differences in the response to food might be explained by differences in the gut microbiota composition and metabolic phenotype, suggesting that dietary interventions may need to be personalized ${ }^{80}$. Screening of the microbiota profile in combination with the metabolic phenotype before designing a weight-loss program or dietary intervention would be an important strategy to follow in the future. In that way, a better insight in beneficial bacteria and targeted supplements with probiotics or prebiotics can be obtained.

3. It has recently been shown that stool consistency is negatively correlated with species richness, positively associated with the Bacteroidetes/Firmicutes ratio, and linked to Akkermansia and Methanobrevibacter abundance ${ }^{81}$. Stool consistency reflects differences in fecal water content and colon activity, and is an indication for intestinal colon transit time. The associations between stool consistency and species richness and community composition emphasizes the importance of stool consistency and transit time assessment in future studies, since this may influence study outcomes.

4. Although we could not detect an association between the microbiome and insulin sensitivity in the present studies, large metagenome-wide association studies indicated differences in the microbiota compostion between lean, obese and diabetic subjects. Based on the present study, we cannot exclude that antibiotic treatment may have had more impact on host physiology in lean, metabolically healthy individuals. It is, therefore, important for future microbiota-manipulation studies to include a lean healthy control group.

5. Thus far, only animal studies have demonstrated the effects of specific bacterial colonies on epithelial integrity. Although animal data are convincing, it is important to investigate the 'leaky gut' hypothesis in a more extensive manner in humans. In order to target LPS-leakage and colonocyte health in future treatment strategies for obesity and metabolic complications, collection of colon biopsies should be considered to investigate the expression of tight-junction proteins. 


\section{References}

1. Obesity and overweight Fact sheet $\mathrm{N}^{\circ} 3112015$.

2. Trends in adult body-mass index in 200 countries from 1975 to 2014: a pooled analysis of 1698 population-based measurement studies with 19\&\#xb7;2 million participants: Elsevier; 2016. Report No.: 0140-6736.

3. Backhed $F$, Ding $H$, Wang $T$, et al. The gut microbiota as an environmental factor that regulates fat storage. Proceedings of the National Academy of Sciences of the United States of America 2004;101:15718-23.

4. Backhed F, Manchester JK, Semenkovich CF, Gordon Jl. Mechanisms underlying the resistance to diet-induced obesity in germ-free mice. Proceedings of the National Academy of Sciences of the United States of America 2007;104:979-84.

5. Cani PD, Bibiloni $R$, Knauf $C$, et al. Changes in gut microbiota control metabolic endotoxemia-induced inflammation in high-fat diet-induced obesity and diabetes in mice. Diabetes 2008;57:1470-81.

6. Turnbaugh PJ, Ley RE, Mahowald MA, Magrini V, Mardis ER, Gordon JI. An obesity-associated gut microbiome with increased capacity for energy harvest. Nature 2006;444:1027-31.

7. Hwang I, Park YJ, Kim YR, et al. Alteration of gut microbiota by vancomycin and bacitracin improves insulin resistance via glucagon-like peptide 1 in diet-induced obesity. FASEB journal : official publication of the Federation of American Societies for Experimental Biology 2015;29:2397-411.

8. Membrez $M$, Blancher $F$, Jaquet $M$, et al. Gut microbiota modulation with norfloxacin and ampicillin enhances glucose tolerance in mice. FASEB journal : official publication of the Federation of American Societies for Experimental Biology 2008;22:2416-26.

9. Murphy EF, Cotter PD, Hogan A, et al. Divergent metabolic outcomes arising from targeted manipulation of the gut microbiota in diet-induced obesity. Gut 2013;62:220-6.

10. Yap IK, Li JV, Saric J, et al. Metabonomic and microbiological analysis of the dynamic effect of vancomycin-induced gut microbiota modification in the mouse. Journal of proteome research 2008;7:3718-28.

11. Shepherd ML, Ponder MA, Burk AO, Milton SC, Swecker WS, Jr. Fibre digestibility, abundance of faecal bacteria and plasma acetate concentrations in overweight adult mares. Journal of nutritional science 2014;3:e10.

12. Wang M, Radlowski EC, Monaco MH, Fahey GC, Jr., Gaskins HR, Donovan SM. Mode of delivery and early nutrition modulate microbial colonization and fermentation products in neonatal piglets. The Journal of nutrition 2013;143:795803. 
13. Rawls JF, Mahowald MA, Ley RE, Gordon Jl. Reciprocal gut microbiota transplants from zebrafish and mice to germ-free recipients reveal host habitat selection. Cell 2006;127:423-33.

14. Shin SC, Kim SH, You $\mathrm{H}$, et al. Drosophila microbiome modulates host developmental and metabolic homeostasis via insulin signaling. Science 2011;334:670-4.

15. Karlsson FH, Tremaroli V, Nookaew I, et al. Gut metagenome in European women with normal, impaired and diabetic glucose control. Nature 2013;498:99103.

16. Larsen N, Vogensen FK, van den Berg FW, et al. Gut microbiota in human adults with type 2 diabetes differs from non-diabetic adults. PloS one 2010;5:e9085.

17. Qin J, Li Y, Cai Z, et al. A metagenome-wide association study of gut microbiota in type 2 diabetes. Nature 2012;490:55-60.

18. Canfora EE, Jocken JW, Blaak EE. Short-chain fatty acids in control of body weight and insulin sensitivity. Nature reviews Endocrinology 2015;11:577-91.

19. Ridlon JM, Kang DJ, Hylemon PB. Bile salt biotransformations by human intestinal bacteria. Journal of lipid research 2006;47:241-59.

20. Conly JM, Stein K, Worobetz L, Rutledge-Harding S. The contribution of vitamin $\mathrm{K}_{2}$ (menaquinones) produced by the intestinal microflora to human nutritional requirements for vitamin $\mathrm{K}$. The American journal of gastroenterology 1994;89:915-23.

21. Arpaia N, Campbell C, Fan X, et al. Metabolites produced by commensal bacteria promote peripheral regulatory T-cell generation. Nature 2013:504:451-5.

22. Cani PD, Amar J, Iglesias MA, et al. Metabolic endotoxemia initiates obesity and insulin resistance. Diabetes 2007;56:1761-72.

23. Vavassori $P$, Mencarelli A, Renga B, Distrutti E, Fiorucci S. The bile acid receptor FXR is a modulator of intestinal innate immunity. Journal of immunology 2009;183:6251-61.

24. Zhu L, Baker SS, Gill C, et al. Characterization of gut microbiomes in nonalcoholic steatohepatitis (NASH) patients: a connection between endogenous alcohol and NASH. Hepatology 2013;57:601-9.

25. Cox LM, Blaser MJ. Pathways in microbe-induced obesity. Cell metabolism 2013;17:883-94.

26. Tremaroli $\mathrm{V}$, Backhed F. Functional interactions between the gut microbiota and host metabolism. Nature 2012;489:242-9.

27. Kerr CA, Grice DM, Tran CD, et al. Early life events influence whole-of-life metabolic health via gut microflora and gut permeability. Critical reviews in microbiology 2015;41:326-40. 
28. Gonzales M, Pepin J, Frost EH, et al. Faecal pharmacokinetics of orally administered vancomycin in patients with suspected Clostridium difficile infection. BMC infectious diseases 2010;10:363.

29. Vrieze $A$, Out $C$, Fuentes $S$, et al. Impact of oral vancomycin on gut microbiota, bile acid metabolism, and insulin sensitivity. Journal of hepatology 2014;60:82431.

30. Dufour $V$, Millon $L$, Faucher JF, et al. Effects of a short-course of amoxicillin/clavulanic acid on systemic and mucosal immunity in healthy adult humans. International immunopharmacology 2005;5:917-28.

31. Chou CJ, Membrez M, Blancher F. Gut decontamination with norfloxacin and ampicillin enhances insulin sensitivity in mice. Nestle Nutrition workshop series Paediatric programme 2008;62:127-37; discussion 37-40.

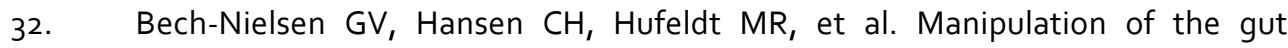
microbiota in $\mathrm{C}_{57} \mathrm{BL} / 6$ mice changes glucose tolerance without affecting weight development and gut mucosal immunity. Research in veterinary science 2012;92:501-8.

33. Carvalho BM, Guadagnini D, Tsukumo DM, et al. Modulation of gut microbiota by antibiotics improves insulin signalling in high-fat fed mice. Diabetologia 2012;55:2823-34.

34. Rune I, Hansen $\mathrm{CH}$, Ellekilde $\mathrm{M}$, et al. Ampicillin-improved glucose tolerance in diet-induced obese $\mathrm{C}_{57} \mathrm{BL} / 6 \mathrm{NTac}$ mice is age dependent. Journal of diabetes research 2013;2013:319321.

35. Rajpal DK, Klein JL, Mayhew D, et al. Selective Spectrum Antibiotic Modulation of the Gut Microbiome in Obesity and Diabetes Rodent Models. PloS one 2015;10:e0145499.

36. Mikkelsen KH, Frost M, Bahl MI, et al. Effect of Antibiotics on Gut Microbiota, Gut Hormones and Glucose Metabolism. PloS one 2015;10:e0142352.

37. Zhang $X$, Shen $D$, Fang $Z$, et al. Human gut microbiota changes reveal the progression of glucose intolerance. PloS one 2013;8:e71108.

38. Ley RE, Backhed F, Turnbaugh P, Lozupone CA, Knight RD, Gordon Jl. Obesity alters gut microbial ecology. Proceedings of the National Academy of Sciences of the United States of America 2005;102:11070-5.

39. Le Poul E, Loison C, Struyf $S$, et al. Functional characterization of human receptors for short chain fatty acids and their role in polymorphonuclear cell activation. The Journal of biological chemistry 2003;278:25481-9.

40. Cox MA, Jackson J, Stanton $M$, et al. Short-chain fatty acids act as antiinflammatory mediators by regulating prostaglandin $\mathrm{E}(2)$ and cytokines. World journal of gastroenterology 2009;15:5549-57. 
41. Furusawa $\mathrm{Y}$, Obata $\mathrm{Y}$, Fukuda $\mathrm{S}$, et al. Commensal microbe-derived butyrate induces the differentiation of colonic regulatory T cells. Nature 2013;504:446-50.

42. Smith PM, Howitt MR, Panikov N, et al. The microbial metabolites, short-chain fatty acids, regulate colonic Treg cell homeostasis. Science 2013;341:569-73.

43. Jones ML, Martoni CJ, Ganopolsky JG, Labbe A, Prakash S. The human microbiome and bile acid metabolism: dysbiosis, dysmetabolism, disease and intervention. Expert opinion on biological therapy 2014;14:467-82.

44. Cani PD, Neyrinck AM, Fava F, et al. Selective increases of bifidobacteria in gut microflora improve high-fat-diet-induced diabetes in mice through a mechanism associated with endotoxaemia. Diabetologia 2007;50:2374-83.

45. Baumgart $M$, Dogan $B$, Rishniw $M$, et al. Culture independent analysis of ileal mucosa reveals a selective increase in invasive Escherichia coli of novel phylogeny relative to depletion of Clostridiales in Crohn's disease involving the ileum. The ISME journal 2007;1:403-18.

46. Frank DN, St Amand AL, Feldman RA, Boedeker EC, Harpaz N, Pace NR. Molecular-phylogenetic characterization of microbial community imbalances in human inflammatory bowel diseases. Proceedings of the National Academy of Sciences of the United States of America 2007;104:13780-5.

47. Lepage $P$, Hasler $R$, Spehlmann $M E$, et al. Twin study indicates loss of interaction between microbiota and mucosa of patients with ulcerative colitis. Gastroenterology 2011;141:227-36.

48. Davie JR. Inhibition of histone deacetylase activity by butyrate. The Journal of nutrition 2003;133:2485S-93S.

49. Ma X, Fan PX, Li LS, Qiao SY, Zhang GL, Li DF. Butyrate promotes the recovering of intestinal wound healing through its positive effect on the tight junctions. Journal of animal science 2012;90 Suppl 4:266-8.

50. Cani PD, Possemiers S, Van de Wiele T, et al. Changes in gut microbiota control inflammation in obese mice through a mechanism involving GLP-2-driven improvement of gut permeability. Gut 2009;58:1091-103.

51. Frazier TH, DiBaise JK, McClain CJ. Gut microbiota, intestinal permeability, obesity-induced inflammation, and liver injury. JPEN Journal of parenteral and enteral nutrition 2011;35:14S-20S.

52. Jernberg C, Lofmark S, Edlund C, Jansson JK. Long-term ecological impacts of antibiotic administration on the human intestinal microbiota. The ISME journal 2007;1:56-66.

53. Dethlefsen L, Huse S, Sogin ML, Relman DA. The pervasive effects of an antibiotic on the human gut microbiota, as revealed by deep 165 rRNA sequencing. PLoS biology 2008;6:e280. 
54. Thuny F, Richet H, Casalta JP, Angelakis E, Habib G, Raoult D. Vancomycin treatment of infective endocarditis is linked with recently acquired obesity. PloS one 2010;5:e9074.

55. Million M, Thuny F, Angelakis E, et al. Lactobacillus reuteri and Escherichia coli in the human gut microbiota may predict weight gain associated with vancomycin treatment. Nutrition \& diabetes 2013;3:e87.

56. David LA, Maurice CF, Carmody RN, et al. Diet rapidly and reproducibly alters the human gut microbiome. Nature 2014;505:559-63.

57. Diamant $M$, Blaak EE, de Vos WM. Do nutrient-gut-microbiota interactions play a role in human obesity, insulin resistance and type 2 diabetes? Obesity reviews : an official journal of the International Association for the Study of Obesity 2011;12:272-81.

58. Delzenne NM, Neyrinck AM, Backhed F, Cani PD. Targeting gut microbiota in obesity: effects of prebiotics and probiotics. Nature reviews Endocrinology 2011;7:639-46.

59. Weickert MO, Mohlig M, Schofl C, et al. Cereal fiber improves whole-body insulin sensitivity in overweight and obese women. Diabetes care 2006;29:77580.

6o. Wahli W, Michalik L. PPARs at the crossroads of lipid signaling and inflammation. Trends in endocrinology and metabolism: TEM 2012;23:351-63.

61. Al-Lahham SH, Roelofsen $\mathrm{H}$, Priebe $\mathrm{M}$, et al. Regulation of adipokine production in human adipose tissue by propionic acid. European journal of clinical investigation 2010;40:401-7.

62. Ge H, Li X, Weiszmann J, et al. Activation of $\mathrm{G}$ protein-coupled receptor 43 in adipocytes leads to inhibition of lipolysis and suppression of plasma free fatty acids. Endocrinology 2008;149:4519-26.

63. Crouse JR, Gerson CD, DeCarli LM, Lieber CS. Role of acetate in the reduction of plasma free fatty acids produced by ethanol in man. Journal of lipid research 1968;9:509-12.

64. Fernandes J, Vogt J, Wolever TM. Intravenous acetate elicits a greater free fatty acid rebound in normal than hyperinsulinaemic humans. European journal of clinical nutrition 2012;66:1029-34.

65. Laurent C, Simoneau C, Marks L, et al. Effect of acetate and propionate on fasting hepatic glucose production in humans. European journal of clinical nutrition 1995;49:484-91.

66. Janssen AW, Kersten S. The role of the gut microbiota in metabolic health. FASEB journal : official publication of the Federation of American Societies for Experimental Biology 2015;29:3111-23. 
67. Goossens $\mathrm{GH}$. The role of adipose tissue dysfunction in the pathogenesis of obesity-related insulin resistance. Physiology \& behavior 2008;94:206-18.

68. Stinkens R, Goossens GH, Jocken JW, Blaak EE. Targeting fatty acid metabolism to improve glucose metabolism. Obesity reviews : an official journal of the International Association for the Study of Obesity 2015;16:715-57.

69. Velagapudi VR, Hezaveh R, Reigstad CS, et al. The gut microbiota modulates host energy and lipid metabolism in mice. Journal of lipid research 2010;51:110112.

70. Couillard C, Mauriege P, Imbeault P, et al. Hyperleptinemia is more closely associated with adipose cell hypertrophy than with adipose tissue hyperplasia. International journal of obesity and related metabolic disorders : journal of the International Association for the Study of Obesity 2000;24:782-8.

71. Thiebaud D, Jacot E, DeFronzo RA, Maeder E, Jequier E, Felber JP. The effect of graded doses of insulin on total glucose uptake, glucose oxidation, and glucose storage in man. Diabetes 1982;31:957-63.

72. Chai W, Dong $Z$, Wang $N$, et al. Glucagon-like peptide 1 recruits microvasculature and increases glucose use in muscle via a nitric oxidedependent mechanism. Diabetes 2012;61:888-96.

73. Cherbut $C$, Ferrier L, Roze C, et al. Short-chain fatty acids modify colonic motility through nerves and polypeptide $Y Y$ release in the rat. The American journal of physiology 1998;275:G1415-22.

74. Bonini JA, Anderson SM, Steiner DF. Molecular cloning and tissue expression of a novel orphan $G$ protein-coupled receptor from rat lung. Biochemical and biophysical research communications 1997;234:190-3.

75. Cornall LM, Mathai ML, Hryciw DH, McAinch AJ. Diet-induced obesity upregulates the abundance of GPR43 and GPR120 in a tissue specific manner. Cellular physiology and biochemistry : international journal of experimental cellular physiology, biochemistry, and pharmacology 2011;28:949-58.

76. Khan MT, Nieuwdorp M, Backhed F. Microbial modulation of insulin sensitivity. Cell metabolism 2014;20:753-60.

77. Dominianni C, Sinha R, Goedert JJ, et al. Sex, body mass index, and dietary fiber intake influence the human gut microbiome. PloS one 2015;10:e0124599.

78. Mueller S, Saunier K, Hanisch C, et al. Differences in fecal microbiota in different European study populations in relation to age, gender, and country: a crosssectional study. Applied and environmental microbiology 2006;72:1027-33.

79. Bolnick DI, Snowberg LK, Hirsch PE, et al. Individual diet has sex-dependent effects on vertebrate gut microbiota. Nature communications 2014;5:4500.

8o. Zeevi D, Korem T, Zmora N, et al. Personalized Nutrition by Prediction of Glycemic Responses. Cell 2015;163:1079-94. 
81. Vandeputte D, Falony G, Vieira-Silva S, Tito RY, Joossens M, Raes J. Stool consistency is strongly associated with gut microbiota richness and composition, enterotypes and bacterial growth rates. Gut 2016;65:57-62. 
152 


\section{ADDENDUM}

Valorization 
The prevalence of obesity has reached epidemic proportions, and affects all ages and socioeconomic groups in the Western world ${ }^{1,2}$. In 2014, $13.1 \%$ of men and $19.9 \%$ of all women were overweight or obese. This worldwide obesity prevalence is expected to reach $18 \%$ in men and to surpass $21 \%$ in women in 2025. The excessive accumulation of body fat in the overweight and obese state increases the risk for the development of type 2 diabetes mellitus ${ }^{3}$, cardiovascular disease, mental disorders ${ }^{4}$ and several types of cancer ${ }^{5}$. The WHO Global Report on Diabetes 2016 showed that the total burden of deaths from high blood glucose has been estimated 3.7 million, including 1.5 million deaths due to diabetes alone. Yet, the rising obesity prevalence is also observed in many low- and middle-income countries. For example, Africa, which is home to 54 lowand middle-income countries, is expected to have the world's largest increase in the death rate caused by chronic diseases over the next decade ${ }^{6}$.

The increasing worldwide prevalence of obesity and related cardiometabolic complications has a considerable economic and social impact. Health care costs include an expensive array of treatments. To delay disease progression, millions of individuals depend on medication and surgery to treat obesity-related complications. For example, medication to lower cholesterol, blood pressure and blood glucose, gastric bypass surgery or cardiovascular surgery. Despite improved medical and technological advances, a downward trend in longevity has been observed that is related to the increased incidence of obesity ${ }^{7}$. Moreover, this decrease in life expectancy might only accelerate in the future, since the current generation of children has a higher BMI and prevalence of related complications than ever before ${ }^{1}$.

It is therefore important to implement new strategies to improve worldwide health and awareness of the problems that come along with being overweight and obese. For years, the public health approach to obesity was the advice to consume less and exercise more. However, attempts to lower body mass for a longer time period without the implementation of a healthy lifestyle in daily life and without addressing biological drivers of weight gain, inevitably fails for the majority of people. Therefore, better knowledge on weight loss strategies, healthy dietary components, and parameters of human metabolism as well as the triggers that may give lead to implementation of a healthy lifestyle are needed and warrant a close collaboration between research, industry and the government. 


\section{Research}

The balance between energy intake, energy expenditure and energy excretion is maintained by a large regulatory network between metabolic organs. There is close interaction between the adipose tissue, skeletal muscle, pancreas and the liver in the regulation of energy and substrate metabolism. Studies performed over the last decade have indicated that the bacteria residing in the intestinal tract are part of this inter-organ crosstalk. Perturbations of microbial populations may contribute to an obesogenic phenotype, inflammation, alterations in glucose metabolism and insulin resistance. Importantly, however, mechanistic studies almost exclusively have been performed in rodents, and results from these studies cannot directly be used to optimize public health guidelines. This thesis describes a clinical trial in overweight and obese humans to better understand if and how specific microbial populations may contribute to the development of obesity and related metabolic and inflammatory perturbations, thereby making scientific knowledge suitable and available for societal or economic utilization. Our findings demonstrate that pronounced modulation of the gut microbiota composition did not significantly affect host metabolism. Even after eight weeks follow-up, when the microbiota composition was still considerably altered as compared to baseline, no significant differences in host metabolism were found. The extrapolation of findings from animal studies to relevance for humans is an important step in the process of valorization. Knowledge on the effect of microbiota manipulation in humans may provide useful information to develop and/or optimize (nutritional or lifestyle-related) strategies to prevent obesity and obesity-related metabolic disturbances. Moreover, the studies described in this thesis provide new perspectives for future research and suggestions for improvement of intervention protocols to further investigate the link between microbiota and human metabolism. For example, since we found large inter-individual differences in the gut microbiota composition and the relationship with metabolic phenotype, this suggests that dietary interventions may need to be more personalized. In addition, future studies may consider inclusion of a lean, metabolically healthy control group. Finally, it is important that future studies include both men and women, since increasing evidence indicates gender differences in physiology, microbiota composition and sex hormones regarding the susceptibility to intervention effects. 


\section{Industry}

The research as described in the present thesis was part of an interdisciplinary collaboration between academia, industry, and government within the framework of the Top Institute Food and Nutrition (TIFN). The ambition of this private-public partnership is to provide the knowledge base that is needed for high-impact innovations in food and nutrition. The motto 'scientific excellence, industrial relevance' indicates the importance of valorization within TIFN. The close collaboration between industry and academia leads to demand-driven research, which might provide long-term business strategies. Although the industrial partners had no leading role in the design of this study, their critical questions and suggestions were taken into account to optimize the study design. The goal of the study as described in this thesis was to provide the nutritional industry with insights in the importance of the intestinal microbiome in obesity and related metabolic impairments. The fact that we were not able to confirm a clear relationship between the gut microbiota and metabolic health parameters in an overweight/obese population shows that the human organism and its interaction with the environment is extremely complex. Inter-individual differences in lifestyle, diet, country of origin, mode of delivery at birth, medical history, travel habits and cultural habits make it hard to determine the exact role of the microbiome in obesity and comorbidities. Long term, moderate alterations of the microbiota composition (e.g. by prebiotics or probiotics) might be needed to obtain health benefits, if the gut microbiota appears to contribute to metabolic health in humans.

\section{Government}

The government may take measures to make the implementation of a healthy lifestyle more feasible, such as taxes on unhealthy products, creating an environment that stimulates physical activity, and the implementation of a healthy lifestyle into health care prevention. The studies described in this thesis are an important first step in elucidating the importance of the gut microbiota in host metabolism in humans, but do not provide the government with direct advice to change policy on guidelines for healthy nutrition. However, these studies show that we are still in an early phase regarding microbiota research, and governmental funding in this area of research remains important to answer a broad range of new questions. 


\section{Health care professionals}

Although the results of the studies as described in this thesis show that microbiota manipulation by seven days antibiotics treatment does not affect human metabolism, the use of antibiotics should be decreased in common practice because of rising antibiotics resistance worldwide. In addition to the increasing problems regarding antbiotics resistance, it has been demonstrated that the risk of developing type 2 diabetes was increased when subjects have been exposed to $>5$ antibiotic treatments ${ }^{8}$. Moreover, the number of prescriptions and duration of antibiotic treatment may accelerate the ageingrelated decline of intestinal integrity ${ }^{9}$. Health care professionals play an important role in encouraging healthy behavior and should provide dietary guidelines to obese patients, involving improved quality of food. The results of the studies described in this thesis do not provide direct guidelines for health care, but indicate that the promising animal data in the field of the gut microbiome generated and published over the last decade, including fecal transplantation data, should be critically evaluated and requires more studies in humans before developing or implementing treatment strategies for common practice.

\section{General public}

When it comes to trust the information that is provided to the general public, scientists appear to be the most trusted, whereas the least trusted group consists of politicians ${ }^{10}$. It is for the scientific community therefore extremely important to provide the general public with clear and understandable information regarding study results. The overwhelming load of information about 'healthy' and 'dangerous' food in the news and social media, often provides people with the wrong information and lifestyle advice. Moreover, the interpretation of scientific results by journalists often needs more consideration.

This thesis shows for the first time that care should be taken with all information available that considers the gut microbiota as the holy grail and, related to that, probiotic drinks as the holy wine. We are far from knowing what the exact role of the microbiota composition and functionality is. However, the large amount of information available within scientific literature indicates that the gut microbiota, as part of an important digestive organ, might differently affect metabolism dependent on metabolic phenotype, age and gender. It is the task of the 
scientific community to elucidate these differences, and investigate whether long term, but moderate dietary or lifestyle manipulations can positively affect body weight and metabolic profile in overweight and obese humans, and may beneficially alter related cardio-metabolic complications.

\section{Concluding remarks}

The study results as described in this thesis have been incorporated in original articles that have been published or submitted to scientific journals in the field of metabolism, diabetes and gastroenterology. These research articles are accessible to scientists who are interested in this topic. In addition, results have been presented at several (inter)national conferences to colleagues inside and outside the specific field, and have been discussed within the TIFN-framework with partners from industry. This study shows once again that care should be taken to extrapolate animal data to guidelines for humans, and that the human organism and its interaction with the environment is extremely complex. 


\section{References}

1. Obesity and overweight Fact sheet $\mathrm{N}^{\circ} 3112015$.

2. Hill JO. Understanding and addressing the epidemic of obesity: an energy balance perspective. Endocrine reviews 2006;27:750-61.

3. Guilherme A, Virbasius JV, Puri V, Czech MP. Adipocyte dysfunctions linking obesity to insulin resistance and type 2 diabetes. Nature reviews Molecular cell biology 2008;9:36777.

4. Preiss K, Brennan L, Clarke D. A systematic review of variables associated with the relationship between obesity and depression. Obesity reviews : an official journal of the International Association for the Study of Obesity 2013;14:906-18.

5. Renehan AG, Zwahlen M, Egger M. Adiposity and cancer risk: new mechanistic insights from epidemiology. Nature reviews Cancer 2015;15:484-98.

6. Naik R, Kaneda T. Noncommunicable diseases in Africa Population Reference Bureau; 2015.

7. Murray CJ, Atkinson C, Bhalla K, et al. The state of US health, 1990-2010: burden of diseases, injuries, and risk factors. Jama 2013;310:591-608.

8. Mikkelsen KH, Knop FK, Frost M, Hallas J, Pottegard A. Use of Antibiotics and Risk of Type 2 Diabetes: A Population-Based Case-Control Study. The Journal of clinical endocrinology and metabolism 2015;100:3633-40.

9. Kerr CA, Grice DM, Tran CD, et al. Early life events influence whole-of-life metabolic health via gut microflora and gut permeability. Critical reviews in microbiology 2015;41:326-40.

10. Ipsos MORI Veracity Index 2015: Trust in Professions United Kingdom: Ipsos Mori Market Research; 2015. 
Summary 
Over the last decades, the prevalence of obesity has reached epidemic proportions, with an increasing impact on health care. Several recent studies have indicated that the composition of the gut microbiota might play a prominent role in body weight regulation and impairments in metabolic health. Moreover, perturbations in microbial populations may contribute to changes in body weight, inflammation, glucose metabolism and insulin sensitivity. Importantly, however, mechanistic studies almost exclusively have been performed in rodent models, and most human evidence comes from large metagenomic studies. Therefore, the studies described in this thesis investigated the impact of manipulation of the gut microbiota composition on human physiology in subjects at increased risk for developing type 2 diabetes mellitus. In addition, we examined the relationship between the microbiota composition at the genus level and tissue-specific insulin sensitivity, as determined by the goldstandard hyperinsulinemic-euglycemic clamp.

In Chapter 2, we demonstrated that a seven-day intervention with amoxicillin (AMOX; broad-spectrum) or vancomycin (VANCO; narrow-spectrum antibiotic) did not affect host metabolism as compared to placebo in 57 obese, insulin resistant males. In this double-blind, randomized placebo-controlled trial, we found a pronounced decrease in bacterial diversity and a reduction of Firmicutesabundance after VANCO, but not after AMOX. The reduction of Firmicutes resulted in decreased plasma and/or fecal concentrations of short-chain fatty acids and bile acid metabolites. However, this did not affect hepatic, peripheral and adipose tissue insulin sensitivity, as determined by a two-step hyperinsulinemic-euglycemic clamp with [6,6-2 $\left.\mathrm{H}_{2}\right]$-glucose tracer infusion. In addition, gut permeability, circulating lipopolysaccharide binding protein (LPB) concentrations and fecal energy harvest were not affected by either VANCO or AMOX.

Despite a still considerably altered microbial composition, also at eight-weeks follow-up, we did not find effects on several aspects of human metabolism such as energy harvest, adipocyte size and whole-body insulin sensitivity.

Additionally, antibiotics intervention did not affect fasting and/or postprandial energy expenditure, substrate utilization and circulating insulin, GLP-1, ANGPTL4 and leptin. In Chapter 3, we studied the effect of a seven-day antibiotics intervention on forearm skeletal muscle metabolism in more detail by 
combining measurements of arterio-venous concentration differences across forearm muscle and forearm blood flow. In line with Chapter 2, antibiotic treatment did neither affect fasting and postprandial plasma glucose, triacylglycerol (TAG) and free fatty acid (FFA) concentrations, nor the extraction of glucose, TAG and FFA across the forearm muscle. In addition, no changes in lactate flux across forearm muscle were observed.

As described in Chapter 2, microarray analysis showed that VANCO induced a significant decrease of immunity-related pathways in adipose tissue. However, these gene expression alterations did not translate into changes in systemic lowgrade inflammatory markers. Both VANCO and AMOX increased adipose tissue gene expression of pathways related to oxidative metabolism and pathways feeding into these cycles, although adipocyte morphology remained unaltered. In addition, in Chapter 4, we performed functional measurements at the level of adipose tissue. Here, we found that seven-day VANCO treatment slightly but significantly improved ex vivo $\beta$-adrenergic sensitivity. However, the physiological importance of this slight effect remains to be determined since no significant effects on ex vivo basal lipolysis and maximal $\beta$-adenergic receptormediated lipolysis in mature human adipocytes were observed. In line, $\beta$ adrenoceptor expression and post-receptor signaling pathways in adipose tissue were unaltered following antibiotics treatment.

Finally, in Chapter 5, we have investigated the relationship between gut microbiota composition and tissue-specific insulin sensitivity in detail in two independent cohorts of obese men (recruited in Maastricht and Amsterdam). Except for the association between centrain bacterial species and $\mathrm{HbA1C}$ in the Maastricht cohort, we did not find any significant associations between fasting and clamp-derived measures of tissue-specific insulin sensitivity in both cohorts.

In conclusion, although previous animal studies have suggested that the gut microbiota may be a promising target to improve metabolic health, our data demonstrated that short-term manipulation of the human gut microbiota by antibiotics does not have any metabolic consequences. A seven-day treatment with VANCO but not AMOX significantly decreased the microbiota diversity and reduced the abundance of Firmicutes, which was accompanied reduced bile acid metabolites and short-chain fatty acids in plasma and/or feces. This did not translate into significant effects on host metabolism directly after cessation of 
the intervention, nor at eight-weeks follow-up, despite the fact that microbiota composition was not recovered completely to baseline at follow-up. In addition, we did not find a significant association between the gut microbiota and the host metabolic phenotype in humans in a cross-sectional analysis in two independent cohorts. Taken together, this thesis does not provide evidence that altering the gut microbiota in a robust, short-term manner has a pronounced impact on host metabolism in obese subjects with impaired glucose metabolism. Interference with the complex relation between the human being and the environment might need other strategies in order to obtain health benefits. 
Samenvatting 
De prevalentie van obesitas is de afgelopen decennia enorm toegenomen, wat geleid heeft tot een toegenomen druk op de gezondheidszorg en gerelateerde kosten. Recent onderzoek heeft aangetoond dat de samenstelling van de populatie darmbacteriën een prominente rol kan spelen in de regulatie van het lichaamsgewicht en het metabolisme. Verstoringen van de bacteriesamenstelling, ofwel microbiota-compositie, kunnen leiden tot veranderingen van het lichaamsgewicht, ontstekingsparameters, de suikerstofwisseling en insulinegevoeligheid. Studies die de onderliggende mechanismen in de mens hebben onderzocht zijn schaars, omdat het merendeel van de studies is vitgevoerd in dieren.

In de onderzoeken die zijn beschreven in dit proefschrift onderzochten we wat het effect was van het manipuleren van de microbiota-compositie op de stofwisseling in mensen met overgewicht en obesitas. Daarnaast onderzochten we de relatie tussen de microbiota en insulinegevoeligheid van de lever, spier en het vetweefsel, organen die een belangrijke rol spelen bij verstoringen in de stofwisseling die aanwezig zijn bij mensen met obesitas en type 2 diabetes mellitus. De insulinegevoeligheid hebben we gemeten met behulp van de hyperinsulinemische-euglycemische clamp, wat de gouden-standaard techniek is.

In Hoofdstuk 2 hebben we aangetoond dat een interventie met amoxicilline (AMOX; breed-spectrum antibioticum) en vancomycine (VANCO; smal-spectrum antibioticum, gericht tegen Gram-positieve bacteriën) geen effect heeft op het metabolisme bij mensen met overgewicht of obesitas. Aan dit onderzoek namen 57 insulineresistente mannen met overgewicht of obesitas deel, die allen gedurende 7 dagen één van de antibiotica of een placebo gebruikten. Dit dubbelblinde, gerandomiseerde onderzoek toonde aan dat de diversiteit van darmbacteriën na VANCO-behandeling was afgenomen, net als de aanwezigheid van de bacteriestam 'Firmicuten'. De AMOX-behandelde groep toonde deze effecten niet.

De afname van Firmicuten resulteerde in een verlaging van plasma en/of feces concentraties van korte-keten vetzuren en galzoutmetabolieten. Dit leidde echter niet tot veranderingen van de insulinegevoeligheid van de lever, spier of vetweefsel (gemeten met de twee-stap hyperinsulinemische-euglycemische clamp met een $\left[6,6-{ }^{2} \mathrm{H}_{2}\right]$-glucose-tracer). Ook de doorlaatbaarheid van de 
darmwand en het inflammatoir profiel, zoals gereflecteerd door circulerende concentraties van LBP en inflammatoire cytokines, bleven onveranderd.

Ondanks dat de microbiota compositie na acht weken follow-up nog niet volledig hersteld was, vonden we ook aan het einde van deze periode geen significante effecten op het metabolisme (energie-opname in de darm, de grootte van vetcellen en de insulinegevoeligheid (HOMA-IR) bij deze mensen met overgewicht en obesitas.

Verder onderzochten we of de interventie met AMOX of VANCO een effect had op het energiegebruik en de vet- en koolhydraatverbranding na een nacht vasten en na een vetrijke maaltijd in de vorm van een milkshake. Ook werden veranderingen in circulerende hormonen zoals insuline, GLP-1, ANGPTL4 en leptine onderzocht. We vonden geen veranderingen in deze hormonen na de interventie. De resultaten beschreven in Hoofdstuk 3 zijn hiermee in overeenstemming. In dit hoofdstuk werd gekeken naar de stofwisseling van de spier in de onderarm met behulp van metingen van arteriële-veneuze concentratieverschillen en doorbloedingsmetingen van de onderarm. Dit onderzoek toonde aan dat antibiotica-behandeling geen significant effect had op de plasma glucose-, vrije-vetzuren- en lactaatconcentraties en de opname van glucose en lipiden door de spier, zowel na een nacht vasten als na het eten van een vetrijke maaltijd.

Zoals beschreven in Hoofdstuk 2, verlaagde behandeling met VANCO de expressie van genen gerelateerd aan het immunsysteem in het vetweefsel. Deze genexpressie veranderingen vertaalden zich echter niet in veranderingen van inflammatoire parameters in het bloed. Zowel VANCO als AMOX verhoogden de expressie van genen die betrokken zijn bij een verhoogde verbranding van koolhydraten en vetten, maar hadden beide geen effect op de vetcelgrootte.

In Hoofdstuk $\mathbf{4}$ hebben we functionele metingen verricht op het niveau van het vetweefsel (ex vivo). We vonden dat behandeling met VANCO gedurende 7 dagen de $\beta$-adrenerge gevoeligheid voor stimulatie van de lipolyse (vetafbraak) in vetcellen enigszins verhoogde. De fysiologische relevantie van dit effect vereist echter verder onderzoek, mede omdat er geen effect gevonden werd op de basale en $\beta$-adenerge recptor-gemedieerde stimulatie van de lipolyse. 
Bovendien bleken de expressie van de $\beta$-adrenoceptor alsmede post-receptor effecten onveranderd na de interventie.

Tot slot hebben we in Hoofdstuk $\mathbf{5}$ de relatie tussen de microbiota samenstelling en de weefselspecifieke insulinegevoeligheid onderzocht in twee onafhankelijke cohorten van mannen met obesitas. De cohorten waren gerekruteerd in Maastricht en Amsterdam. Met uitzondering van de associatie tussen bepaalde bacteriën en $\mathrm{HbA} 1 \mathrm{c}$, een maat voor de regulatie van de glucose spiegel over een langere periode, in het Maastricht-cohort, vonden we geen enkele significante associatie tussen de microbiota en insulinegevoeligheid in beide cohorten, bepaald aan de hand van zowel gevaste parameters in het bloed en de insulinegevoeligheid zoals gemeten met behulp van de gouden-standaard techniek.

Concluderend kunnen we zeggen dat, in tegenstelling tot dierstudies die suggereerden dat de microbiota een grote rol speelt bij het metabolisme, wij geen relatie vonden tussen de bacteriesamenstelling in de darm en de stofwisseling bij mannem et overgewicht en obesitas. Behandeling met VANCO, maar niet AMOX, gedurende zeven dagen verlaagde de diversiteit van de microbiota en de aanwezigheid van de bacteriestam Firmicuten. Dit ging gepaard met een afname van galzoutmetabolieten en korte-keten vetzuren in de plasma en/of feces, maar vertaalde zich niet in een significant effect op het metabolisme van de gastheer. Ook twee maanden na het stoppen van de antibiotica vonden we geen aantoonbare effecten op de insulinegevoeligheid, ondanks het feit dat de microbiota nog steeds niet volledig hersteld was naar de samenstelling zoals aan het begin van het onderzoek.

Kortom, de onderzoeken beschreven in dit proefschrift tonen aan dat het veranderen van de darmbacterie-samenstelling met antibiotica op korte termijn geen significante effecten heeft op de stofwisseling in mensen met overgewicht en obesitas. Andere strategieën lijken noodzakelijk om de complexe relatie tussen de mens en zijn omgeving te manipuleren om de metabole gezondheid te bevorderen. 
Curriculum Vitae 
Dorien Reijnders was born on July $10^{\text {th }} 1988$ in Heerlen, The Netherlands. She completed secondary school at the Sintermeerten College in Heerlen in 2006. Consecutively, she studied Biomedical Sciences at the Radboud University in Nijmegen, The Netherlands. She combined the Masters Toxicology and Pathobiology. As part of the Toxicology Master, she visited the Toxicology lab at the Karolinska Institute in Stockholm, Sweden, for a 6-month internship to perform experiments related to cell cycle mechanisms in 2010. During the Pathology Master in 2011, she investigated epithelial-mesenchymal transition of kidney cells during a 6-month interniship at the Department of Toxicology and Pharmacology in Nijmegen.

In September 2011, she started her PhD research at the Department of Human Biology at Maastricht University (NUTRIM School of Nutrition and Translational Research in Metabolism), under supervision of Prof. Ellen E. Blaak and Dr. Gijs H. Goossens. Her research, which is described in this thesis, entiteld 'Microbiota, Obesity and Insulin Resistance - Unraveling the impact of the microbiome on metabolic health in humans', was performed within the framework of the Top Institute Food and Nutrition (TIFN). Dorien has presented these research findings at several national conferences, the $22^{\text {th }}$ European Congress on Obesity in Prague, Czech Republic (2015), and the European Molecular Biology Laboratory (EMBL) conference in Heidelberg, Germany, in 2015. 


\section{List of Publications}


Effects of Gut Microbiota Manipulation by Antibiotics on Host Metabolism in Obese Humans: A Randomized Double-Blind Placebo-Controlled Trial.

Reijnders D, Goossens GH, Hermes GD, Neis EP, van der Beek CM, Most J, Holst JJ, Lenaerts K, Kootte RS, Nieuwdorp M, Groen AK, Olde Damink SW, Boekschoten MV, Smidt H, Zoetendal EG, Dejong $\mathrm{CH}$, Blaak EE.

Cell Metabolism. $2016 \mathrm{Jul}$ 12;24(1):63-74.

Effects of amoxicillin and vancomycin on skeletal muscle substrate metabolism in obese men: a randomized controlled trial

Reijnders D, Goossens GH, Blaak EE

Submitted

Gut microbiota composition strongly correlates to peripheral insulin sensitivity in obese men but not in women

Most J, Goossens GH, Reijnders D, Canfora EE, Penders J, Blaak EE

Submitted

Gut microbiota manipulation and lipolysis in human adipose tissue

Reijnders D, Jocken JWE, Canfora EE, Plat J, Goossens GH, Blaak EE

To be submitted

Microbiota composition is not correlated to insulin sensitivity, but represents distinct alternative dysbiotic states in two independent cohorts of overweight and obese males

Reijnders D, Hermes GD, Kootte RS, Goossens GH, Smidt H, Nieuwdorp M, Zoetendal EG, Blaak EE

To be submitted

Impact of oral vancomycin on gut microbiota, bile acid metabolism, and insulin sensitivity.

Vrieze A, Out C, Fuentes S, Jonker L, Reuling I, Kootte RS, van Nood E, Holleman F, Knaapen M, Romijn JA, Soeters MR, Blaak EE, Dallinga-Thie GM, Reijnders $D$, Ackermans MT, Serlie MJ, Knop FK, Holst JJ, van der Ley C, Kema IP, Zoetendal EG, de Vos WM, Hoekstra JB, Stroes ES, Groen AK, Nieuwdorp M.

Journal of Hepatology. 2014 Apr;6o(4):824-31. 
5-Fluorouracil-induced RNA stress engages a TRAIL-DISC-dependent apoptosis axis facilitated by $\mathrm{p} 53$.

Akpinar B, Bracht EV, Reijnders D, Safarikova B, Jelinkova I, Grandien A, Vaculova AH, Zhivotovsky B, Olsson M.

Oncotarget. 2015 Dec 22;6(41):43679-97.

Uremic toxins inhibit renal metabolic capacity through interference with glucuronidation and mitochondrial respiration.

Mutsaers HA, Wilmer MJ, Reijnders D, Jansen J, van den Broek PH, Forkink M, Schepers E, Glorieux G, Vanholder R, van den Heuvel LP, Hoenderop JG, Masereeuw R. Biochimica Biophysica Acta. 2013 Jan;1832(1):142-50.

Role of p-glycoprotein expression and function in cystinotic renal proximal tubular cells.

Peeters K, Wilmer MJ, Schoeber JP, Reijnders D, Heuvel LP, Masereeuw R, Levtchenko E. Pharmaceutics. 2011 Oct 27;3(4):782-92. 
174 
Dankwoord 
Ten eerste zou graag de leden van mijn beoordelingscommissie bedanken voor de tijd en moeite die zij genomen hebben mijn proefschrift te beoordelen. Prof. Marleen van Baak, Dr. John Penders, Prof. Ko Willems-van Dijk en prof. Ad Masclee, bedankt! Prof. Adré Marette, I sincerely appreciate that you take time to visit Maastricht for my thesis defense, thank you!

Ellen, dankjewel dat je ooit de redelijk onervaren Limburgse toxicologiestudente hebt aangenomen die absoluut niet in de Randstad wilde werken. Ik dacht dat ik het vergeten kon toen ik er achter kwam dat jij uit Rotterdam kwam ;) Ellen, je geduld is eindeloos, en je geruststellend vermogen groot. Je kon met een paar woorden mijn stress weer wat reduceren. Je hebt me talloze keren er aan moeten herinneren dat mijn onderzoek echt wel veel waard was. Ik bewonder hoeveel zaken je tegelijk kunt voorzitten en alsnog tijd hebt naar onze papers te kijken. Dankjewel voor deze leerzame, maar ook gezellige jaren waarin de deur altijd open stond!

Gijs, ook al heb ik me vaak verbaasd over hoe jij elk willekeurig stuk tekst van een regenboog aan comments kan voorzien, het werd er wel beter op. Ik moet het toch echt toegeven ;) Ik wil je bedanken voor de toewijding en inzet bij mijn project! Ik hoefde maar binnen te lopen en je luisterde (en praatte haha), en ook jij kon alles goed relativeren. Buiten het werk ben je een mega gezellig persoon, in Barcelona tot laat in de nacht mee op stap, en dansen op de NVDO. Buiten dat dat gewoon heel gezellig was, heb ik altijd erg gewaardeerd dat je óók aan zo een activiteiten belang hechtte! Dankjewel!

Dan wil ik graag mijn co-auteurs bedanken voor hun input en commentaren om de papers in dit proefschrift tot een mooi, helder verhaal te maken. Erwin en Hauke, sorry voor jullie volle inbox. Gerben, jij hebt het laatste half jaar veel op je bord gekregen door ons, bedankt voor je geduld! Ruud, ook jij bedankt voor je snelle reacties, ondanks je opleidingsplek had je toch nog tijd je bezig te houden met onze papers. Max, dank voor je altijd positieve woorden. Johan, je hebt heel wat tijd aan de in vitro-experimenten besteed, en ook aan het schrijven van het paper. Heel erg bedankt daar voor! 
Dit proefschrift was niet tot stand gekomen door de vrijwillige deelname van mijn proefpersonen. Ik besef dat ik veel van jullie heb gevraagd...bloed, urine, feces, vet, spier. Voor mij routine, voor jullie toch vreemd. Ik dank jullie dat jullie voor de ongecompliceerde deelname, en dat jullie allemaal de studie hebben afgemaakt. Wat leer je veel over iemand als je 6 dagen samen in een onderzoekskamer zit!

Ook mijn studenten wil ik graag bedanken voor de hulp op testdagen en de duizenden cupjes die jullie geplakt hebben. Ohja, en het afwegen van de feces niet te vergeten ;) Farah, Rory, Hein, Caspar en Larissa, thanks!

Mijn collega's van HB/BW. Ik heb mijn tijd bij HB als een fantastische tijd ervaren, maar sinds mijn verblijf in de VS weet ik de HB/BW-familie nog meer te waarderen! Wie gaat er nu 2 keer per jaar een weekend weg met zijn collega's? Congressen werden topvakanties, ambtenarencarnaval werd geschminkt en kerstborrels werden memorabele feesten. Hulp was daar waar nodig! Ik kan me niet herinneren dat iemand te beroerd was iets voor een ander te doen. De bulk aan foto's die ik van jullie heb laat me af en toe alle mooie momenten herbeleven! Ik mis jullie! Bedankt Nadia, Laura, Oing, Kenneth, Cyril, Freek, Ronny, Mathijs, Guilio, Bernard, Anne, Jan, Mark, Alex, Martine, Sophie, Peter, Eva, Lena, Bram, Marlies, Dirk, Emmani, Hannah, Lisje, Esther, Charlotte, Lotte, Stefan G, Evelien B, Andy, Imre, Marlou, Jean \& Joey. En de 'oude' garde: Stefan C, Rick, Sanne V, Sanne vd M, Erik, Anouk, Boris, Ellen K, Maarten, Guy V en Annemieke!

\section{Een speciaal woord van dank aan:}

Mijn roomies, Manu en Jasper, mijn gekke Duitsers, met wie feestjes ook echt een feest werden, die me geleerd hebben om tot laat in de nacht door te gaan en een Jagermeister extra te drinken. Jullie hebben mede voor gezorgd hebben dat H.0.348 als mijn 2e thuis voelde. Met cadeautjes voor Kerst, Sinterklaas en verjaardagen. Afgelopen jaar is er nog een gekke Duitse bij gekomen ;) Mattea, ich dachte, dass es komisch sein wird, wenn jemand anderes als Jasper neben mir sitzen soll, aber schon ab dem ersten Tag hat es super gepasst. Auch du weisst wie man eine Party feiert! Weihnachten, Prag, Geburtstagsfeier, Cocktails trinken und die HB Wochenenden waren immer Spass wenn du dabei warst! 
Danke fur die schöne Zeit und so ein liebe Kollegin zu sein! Viel Erfolg mit deinen Studien!

Birgitta, Rudi, Max en Roel, ten eerste dankjulliewel omdat jullie ook allemaal in zekere mate hebben bijgedragen aan mijn studie. Simultane bloedafnames bij de mealtest, dubbele clamps, ik hoefde maar een kik te geven en jullie stonden klaar. Daarnaast zijn jullie gewoon hele gezellige collega's, reisgenootjes en stapmaatjes. Stiekem mis ik toch de tijden dat we allemaal om $7 u$ 's ochtends op het MRUM rondrenden! Birgitta, ik kon jou altijd om advies vragen, want je bent rechtuit en eerlijk! Daarnaast ben je ook heel gezellig en betrokken, dankjewel! Ook voor de leuke congressen, zij het soms met slapeloze nachten :p

Guy, mijn allerleukste buurman! Jouw goede bui straal je uit naar anderen! Tanja, jij bent een ontzettend lief en begripvol mens, en hebt altijd de deur open staan! Net als Silvie, Dorien en Sabine, wat heb ik het altijd gezellig gevonden bij jullie even te komen kletsen, jullie zijn altijd optimistisch en waren vaak een heel lief luisterend oor! Jullie ook Pilou, Nuria en Eveline! We moeten snel weer eens samen aan de wijn! $:$;

Jos, es inne wit wie se luuj same brings, bis dich ut waal. Dank vuur dien ongeleufelijke inzet um luuj noa dr borrel te kriege, weekendjes weg te goan en dr kerstmaat in Oake onveilig te make. Sorry dat dea in Luuk nog ummer neet gelukt is! Ut woar ummer e fijn idee dat dr boave nog iemes aanwezig woar, ooch al woar $\mathrm{t}$ laat op dr oavend. Danke vuur dien loesterend oer, interesse, en ut netjes opruume van al mien samples in $\mathrm{dr}$ vreezer $(-)$ En natuurlijk al dien analyses neet te vergeate! Ich goan dich misse!

Antoine, es ich aan dr borrel dink, dink ich ooch aan dich. Inne borrel is ginne goeie es dich neet bis gewea! Doe zurgs vuur de nuudige gezelligheid en optimisme en bis ummer eave geïnteresseerd! Ich ving t ummer erg fijn mit dich te kletjse! Proost op de Gipfelfreunde! Loek, ik hoefde maar te bellen en je stond voor me klaar! Dankjewel voor je hulp, maar ook voor de gezellige praatjes op de borrel of in de trein! Ook een merci aan Paul, die altijd weer kwam opdraven als er iets piepte in de technische ruimte. Joan, jij hebt gouden handen. Je hebt heel wat testdagen gered door het prikken van onmogelijke infusen!

Desiree, Claudia, Yolanda en Cleo, wat moet een afdeling zonder een warm en welkomend secretariaat? Jullie weten alles en kunnen alles, jullie zijn de moeders van de afdeling waar je altijd terecht kunt, ook al is het om even je hart te luchten. Dankjulliewel! 
Gabby, als het om TIFN ging was jij diegene die alles kon oplossen :-) Wendy niet alleen bedankt voor je analyses, maar ook voor het oplossen van problemen met de clamp-apparatuur! Hasibe, Yvonne, Nicole, ik ben jullie erg dankbaar voor het overnemen van heel veel werk, met name de vetcelgrootte-bepalingen! Dat heeft me heel wat stress gescheeld!

Promoveren doe je niet zonder afleiding van goede vrienden. Linda, je bent al meer dan 12 jaar mijn vriendin, dus wat vond ik het leuk dat jij ook in Maastricht bent gebleven! Onze koffiepauzes duurden meestal iets langer dan gepland haha. Net als die met Ivette, Maud en Ankie, dankjulliewel voor de gezelligheid en het delen van lief en leed! :) Ook buiten het werk had ik altijd lieve mensen om me heen. Lieke, Wouter, Denise, Elianne, Kim, Renee, Dorien, Debby, Ralph en Lydia, jullie zullen misschien wel eens moe zijn geworden van mijn verhalen, toch bedankt voor het luisteren! ;) Lieke, Jori, Marion and Karoline, I loved our trips and hope to continue our tradition! Spending the weekend with you and all stress is gone $:$ Thanks!

Dan wil ik ook mijn familie en soort-van-familie niet in dit dankwoord vergeten voor hun interesse en afleiding! Marja en Sjef, ook al is jouw vakgebied totaal niet het mijne, het was toch altijd fijn te weten dat jij als prof weet wat een promotie inhoudt! Fons en Margriet, jullie oprechte interesse en tips zorgen voor motivatie! John, Ans, Toos, Cees, Marie-Jozé, Léon, Yolan en Willem, met jullie is het altijd gezellig, no matter what $:-$ Cuddel, José en de rest van familie Most, dank voor jullie interesse en dat ik me bij jullie thuis kan voelen!

\section{Een extra woordje van dank aan mijn paranimfen:}

Manu, ik vind het super dat je ondanks je eigen promotie-stress en huwelijksvoorbereidingen toch mijn paranimf wil zijn! We zijn samen begonnen en sluiten dit nu samen af met een groot feest! Je bent altijd even geïnteresseerd geweest in mijn project en dagelijkse leven. Ik kan je niet genoeg bedanken voor je medeleven en support. Ik denk dat je je af en toe best wel ongemakkelijk gevoeld moet hebben met Jasper en mij op 1 kamer i) Jouw soms chaotische voorkomen, je neuriën, zingen of hummen heeft me wel eens gek gemaakt, maar inmiddels mis is het wel! Je hebt immers altijd goede zin en best een meester in positief denken! Daarmee ga je heel ver komen (:) 
Kirsten, wat heb ik een leuke vriendin over gehouden aan deze promotie! Onze koffie-dates waren altijd een goed ontspan-moment! Ik vind het super leuk dat je mijn paranimf wil zijn! Je bent altijd heel betrokken geweest bij mijn project en hebt heel wat frustratie moeten aanhoren ;) Ik weet niet of ik het al eens zo duidelijk gezegd heb, maar mijn project was lang niet zo soepel gelopen als jij niet op de meest vreemde tijdstippen bereid was biopten af te nemen! Jou was niks te gek! Of ik je nu om $7 u$ 's ochtends nodig had of 2 biopten tegelijk had lopen, en daar naast nog een clamp deed. Alles kon, en nooit met slechte zin! Ook niet als ik wel eens iets vergeten was klaar te leggen ;) Dankjewel! En die koffie/wijn/sushi-dates pakken we gewoon weer op als ik weer terug uit de VS ben! Heel erg veel succes met je opleiding en promotie!

Pap en Mama, ten eerste natuurlijk bedankt voor jullie support, motivatie en het aanhoren van mijn promotie-verhalen, ook al was daar misschien soms weinig van te begrijpen ;) Bedankt voor de afleiding als ik die hard nodig had, maar nog meer wil ik dankjewel zeggen voor de vrijheid die jullie ons geven. Bij ons thuis kan alles en is niks te gek! Tijdens de periode met testdagen stond er altijd een bord eten klaar, ook al was ik nooit op tijd thuis. Zelfs op vrijdag, als ik standaard vanaf de borrel belde dat ik toch wat langer bleef...En thuis wonen na 10 jaar is best gek, maar bij jullie eigenlijk wel heel erg gezellig! Jack, ik ben heel blij dat ik altijd op zo een creatieveling terug kan vallen als ik iets gedaan moet hebben, ook al is het $6 u$ 's ochtends vlak voordat je op vakantie vertrekt! Dankjewel voor je hulp bij het maken van figuren en kaft! Ik mis mijn broertje wel in de VS!

Lieve Jasper, ik denk niet dat iemand zal ontkennen dat jij de belangrijkste persoon tijdens mijn promotie bent geweest :) Als collega was je de beste die ik wensen kon...ook voor jou was geen moment te gek om een biopt af te nemen. Door jouw flexibiliteit en behulpzaamheid liep mijn studie een stuk vlotter. Je kwam me op drukke dagen aflossen voordat ik zelf een glucose-infuus nodig had, hebt heel wat keren meegeholpen mijn cupjes te sorteren, orde in mijn vriezer geschept en haalde iedere morgen stikstof. Jouw excel-skills zijn fantastisch en jouw templates hebben me heel wat werk gespaard. Naast behulpzame, lieve collega werd je ook mijn beste vriend, die me verraste met een feestje voor mijn laatste van 308 testdagen, muffins voor mijn verjaardag bakte en een peperkoekhuisje bouwde met kerst. En als ik mijn trein weer eens had gemist, 
mocht ik gewoon met jouw auto naar huis! Ik ben heel gelukkig dat ik sinds 2 jaar dagelijks in jouw auto mag rijden, en zou niet weten wat ik nog zonder jou moet. Je hebt alles voor mij over, weet me altijd weer aan het lachen te krijgen als het even tegen zit en laat me zoveel mopperen en gek doen als ik wil! Lieve Japs, je bent de echt de leukste! Ik hou van jou! xxx 